\title{
Macro-invertébrés du Cénomanien du Djebel Metrassi (Batna, NE Algérie): Systématique et biostratigraphie
}

\author{
Macro-invertebrates from the Cenomanian of Djebel \\ Metrassi (Batna, NE Algeria): Systematics \\ and biostratigraphy
}

\author{
R. Aouissi ${ }^{1}$, S. Salmi-Laouar ${ }^{2}$, B. Ferré ${ }^{3}$
}

${ }^{1}$ Université Larbi Ben M'hidi, Oum el Bouaghi, Laboratoire de Recherche de Géologie (LRG), B.P. 12, 23000 Annaba, Algérie. Email: aouissi riadh@hotmail.com https://orcid.org/0000-0003-2083-155X

${ }^{2}$ Université Badji Mokhtar, Annaba, Laboratoire de Recherche de Géologie (LRG), B.P. 12, 23000 Annaba, Algérie. Email: ssalmit@yahoo.fr https://orcid.org/0000-0002-4095-5851

${ }^{3}$ Dame du Lac 213, 3 rue Henri Barbusse, F-76300 Sotteville-lès-Rouen, France. Email: bruno_ferre@yahoo.fr https://orcid. org/0000-0001-5350-9407

\section{RÉSUMÉ}

Un assemblage paléontologique a été récolté dans le Cénomanien du Djebel Metrassi (Batna, NE Algérie). Constitué de 327 spécimens, il comporte un corail, 24 espèces de bivalves, 14 espèces de gastéropodes, 3 espèces d'ammonites et 7 espèces d'échinides. Cet assemblage permet d'établir une liste systématique originale, avec des espèces inédites, pour le Cénomanien de ce Djebel et vient soutenir le découpage lithostratigraphique proposé.

Mots clés: Djebel Metrassi; Batna; Algérie; Cénomanien; macro-invertébrés; systématique; biostratigraphie.

\section{ABSTRACT}

A rich paleontological assemblage was collected in the Cenomanian deposits of the Djebel Metrassi (Batna, NE Algeria). It consists of 327 specimens assigned respectively to a single coral, 24 bivalve, 14 gastropod, 3 ammonite, and 7 echinoid species. This macrofaunal association leads us to establish an original taxonomical reference list along with several species new for the Cenomanian of this area, and supports the lithostratigraphic framework herein proposed.

Keywords: Djebel Metrassi; Batna; Algeria; Cénomanian; Macro-invertebrates; Systematics; Biostratigraphy. 


\section{Introduction}

Dans le Nord-Est algérien, les monts de BatnaBellezma se situent dans la zone de convergence entre le domaine de l'Atlas tellien au Nord et le domaine de l'Atlas saharien au Sud (Bellion, 1972). Limités au Nord-Ouest par les monts de Hodna (Guiraud, 1973), ils héritent conjointement du passé tectonosédimentaire de ces domaines, influencé par la configuration paléo-géographique de la marge méridionale de la Téthys. La macrofaune cénomanienne (coraux, huîtres exogyrines, rudistes, gastéropodes, ammonites et échinides) rencontrée dans la région de Batna est comparable à celle de l'intervalle cénomanien du Tell (Ficheur, 1901), du Constantinois (Coquand, 1862, 1880), du Hodna (Glaçon, 1952; Chikhi-Aouimeur, 2004), de l'Atlas saharien (Benkherouf, 1987; Chikhi-Aouimeur, 1998; Gill \& Chikhi, 1991, Naïli et al., 1995; Ruault-Djerrab, 2012; Slami, 2014; Benyoucef et al., 2017) et même à celle de la plateforme saharienne (Benyoucef et al., 2012, 2015; Zaoui et al., 2016).

La synthèse des études sur les macro-invertébrés de la même période (depuis le Maroc: Lambert, 1932; Petitot, 1961; Freneix, 1972; Meister \& Rhalmi, 2002; Meister et al., 2017; la Tunisie: Pervinquière, 1907, 1912; Zaghbib-Turki, 1989; Meister \& Abdallah, 2005; Kennedy \& Gale, 2015; l'Égypte: AbdelGawad \& Gameil, 1995, 2002; Abdel-Gawad et al., 2004a , 2004b; Gertsch et al., 2008; Ayoub-Hannaa, 2011; Ayoub-Hannaa \& Fürsich, 2011; AyoubHannaa et al., 2014; Pandey et al., 2011; jusqu'en Jordanie: Ahmad \& Al-Hammad, 2002; Aly et al., 2008; Özer \& Ahmad, 2016; et en Oman: Philip et al., 1995) révèle d'étroites similitudes avec les taxons récoltés dans la région de Batna. L'homogénéité de la faune cénomanienne et sa large répartition géographique sud-téthysienne en font un marqueur à affinité paléo-biogéographique permettant des corrélations précises pour les régions nord-africaines.

Ainsi, en domaine téthysien, la présence des ammonites Mantelliceras cf. dixoni et Mantelliceras cantianum marque le Cénomanien inférieur (Robaszynski et al., 1993; Slami, 2014). La vaste distribution géographique et l'extension stratigraphique restreinte font d'Aspidiscus cristatus un bon élément de corrélation pour les parties moyenne à supérieure du Cénomanien pour l'ensemble du bassin méditerranéen
(Gill \& Lafuste, 1987; Gill \& Chikhi, 1991; Pandey et al., 2011). L'ammonite Neolobites vibrayeanus montre une nette tendance à l'endémisme en Afrique du Nord et au Moyen-Orient. Elle est également très commune vers la base du Cénomanien supérieur ibérique (Meister \& Rhalmi, 2002; Meister \& Abdallah, 2005; Wiese \& Schulze, 2005; Aly et al., 2008; Gertsch et al., 2008; Benyoucef et al., 2012, 2017) et permet de préciser la subdivision de l'étage. Les huîtres exogyrines sont typiques des dépôts cénomaniens sud-téthysiens (au Maroc: Freneix, 1972; en Algérie: Coquand, 1862; Busson et al., 1999; Benyoucef et al., 2012; en Tunisie: Pervinquière, 1912; en Libye: Rossi-Ronchetti \& Albanesi, 1961; et en Égypte: Gertsch et al., 2008; Ayoub-Hannaa \& Fürsich, 2011; Ayoub-Hannaa et al., 2014; et Jordanie: Schulze et al., 2005) caractéristiques de mer peu profonde (Dhondt \& Jaillard, 2005). Un assemblage de rudistes (caprinules) est signalé au Maghreb (ChikhiAouimeur et al., 2006), en Algérie (Chikhi-Aouimeur, 1995; Benyoucef et al., 2012), en Égypte (Aly et al., 2005) et en Jordanie (Özer \& Ahmad, 2016). Il permet d'attribuer un âge cénomanien supérieur à ces dépôts.

Les études sur le Cénomanien de la région de Batna sont rares et se limitent à des descriptions lithologiques générales (Savornin, 1931; Bellion, 1972; Vivière, 1985; Yahiaoui, 1990), parfois structurales (Bureau, 1967, 1975, 1986; Vila, 1980), accompagnées d'un recensement paléontologique parfois sommaire (Laffitte, 1939; Bellion, 1972; Benkherouf, 1988; Slami, 2014; Slami et al., 2018). Malgré ces études, les monts de Bellezma-Batna restent mal connus sur le plan paléontologique. Afin de mieux connaître le Cénomanien de la région de Batna, en particulier aux alentours de Metrassi, plusieurs récoltes systématiques ont été réalisées. Ainsi, un assemblage représentatif de la macrofaune d'invertébrés, composé essentiellement de coraux, de bivalves, de gastéropodes, d'ammonites et d'échinides, est réuni et identifié pour la première fois. La présente étude vise à établir une première liste taxonomique et à préciser le cadre stratigraphique de cette macrofaune.

\section{Cadre géologique}

Située au Nord-Est de l'Algérie, la région de Batna appartient aux monts de Bellezma-Batna (Fig. 1). Cette chaîne de montagnes correspond à 


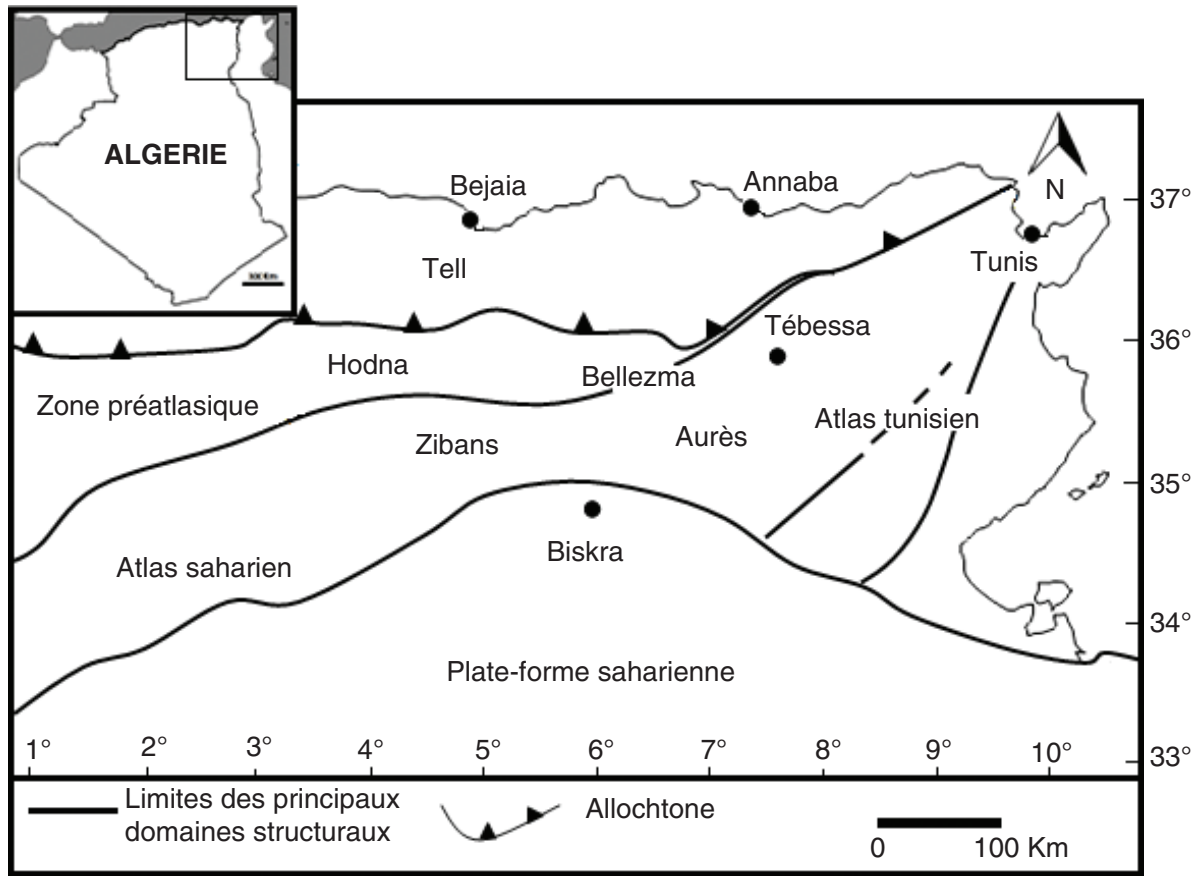

Fig. 1.-Localisation des monts de Bellezma-Batna au sein des grands ensembles géologiques de l'Algérie orientale (Herkat, 2007).

un alignement NE-SO de reliefs, s'étendant de la ville de Batna à l'Est jusqu'au village de Sefiane à l'Ouest. Elle est formée de terrains essentiellement mésozoïques où le Crétacé supérieur affleure sur de vastes étendues (Fig. 2); il est marqué par une richesse en macrofaune bien conservée, surtout dans des niveaux de faciès marins et carbonatés.

Le Djebel Metrassi, objet de cette étude, est situé sur le versant septentrional de l'ensemble nord-est de cette chaîne (cf. Fig. 2) et culminé à 1334 m. Il présente une structure anticlinale dissymétrique de direction $\mathrm{N} 40^{\circ}$ où le flanc sud montre un redressement plus prononcé. Cette structure, affectée par un système de failles de directions NO-SE, $\mathrm{NE}-\mathrm{SO}$ et E-O, hérite des phases tectoniques atlasique et alpine bien connues dans le NE algérien (Dubourdieu \& Durozoy, 1950; Bellion, 1972; Vila, 1980; Kazi-Tani, 1986).

\section{Description lithologique}

L'étude lithostratigraphique du Cénomanien du Djebel Metrassi porte sur une formation lithologique d'extension régionale, épaisse d'environ $260 \mathrm{~m}$, appelée 'Marnes de Smail' (Yahiaoui, 1990). Cette formation est subdivisée en quatre unités (Fig. 3):

\section{Unité A: Marnes à ammonites et exogyres}

Cette unité débute par une vire de marnes argileuses de plus de $100 \mathrm{~m}$ d'épaisseur, de couleur verdâtre à grisâtre, parfois sombres. Ces marnes sont riches en fissures millimétriques remplies de gypse lamellaire d'origine syndiagénétique. Elles renferment une riche macrofaune d'invertébrés dominée par des bivalves (Nucula margaritifera, Pinna sp., Exogyra conica, Costagyra olisiponensis, Ceratostreon flabellatum, Rhynchostreon suborbiculatum, Plicatula auressensis, Plicatula sp., Neithea coquandi, Pterotrigonia scabra, Clisocolus corrugatus, Granocardium carolinum), des gastéropodes (Turritella difficilis, Campanile ganesha, Cimolithium tenouklense, Aporrhais dutrugei, Pterodonta elongata, Tylostoma globosum, et Avellana cassis), des ammonites (Sharpeiceras laticlavium) et des échinides (Heterodiadema libycum, Hemiaster gabrielis, H. pseudofourneli, H. batnensis). Ces marnes sont surmontées par une 


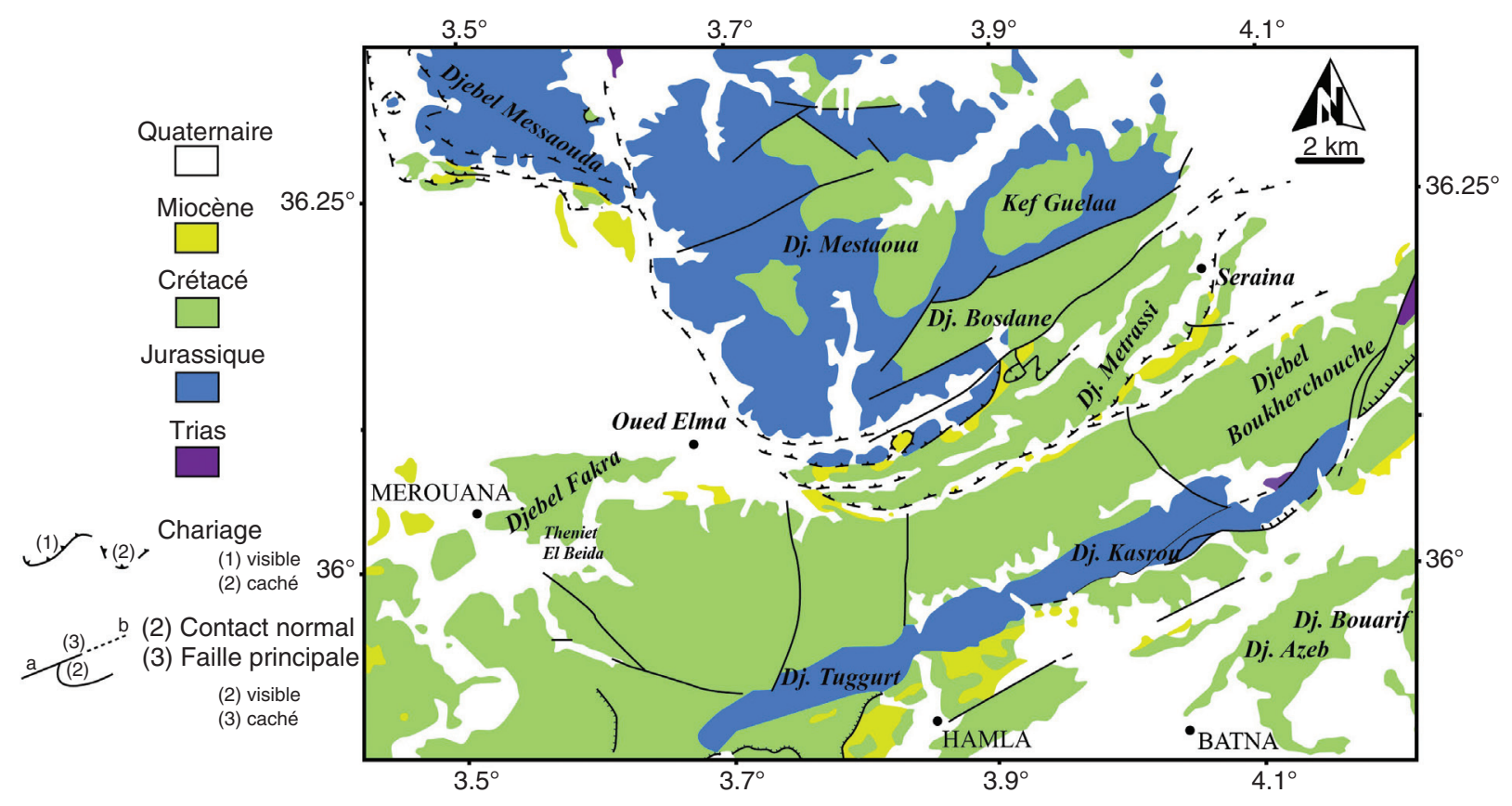

Fig. 2.-Carte géologique simplifiée montrant les formations du Crétacé de Metrassi (carte de Merouana 1/50 000).

alternance de bancs calcaires jaunâtres à texture packstone et de marnes grisâtres, d'une dizaine de mètres d'épaisseur. Ces niveaux sont riches en échinides en bon état de préservation (Hemiaster gabrielis) et en bivalves (Ceratostreon flabellatum). La limite supérieure de cette succession est une surface ferruginisée.

Les dépôts homogènes de cette unité démontrent un milieu stable plus ou moins ouvert et profond, à fond vaseux, situé à la partie distale de la plateforme. La biodiversité élevée suggère plusieurs causes, notamment la richesse du milieu en éléments nutritifs, ou des substrats stables et bien oxygénés, situés dans la zone photique ou sous la limite d'action des vagues.

\section{Unité B: Marnes à Aspidiscus cristatus}

Elle forme une vire marneuse, épaisse de plus de $50 \mathrm{~m}$, très riche en corail attribué à Aspidiscus cristatus. Cette espèce marque le Cénomanien moyen à supérieur (Pomel, 1972; Gill \& Lafuste, 1987; Gill \& Chikhi, 1991; Pandey et al., 2011). Elle est associée à l'ammonite Acanthoceras rhotomagense, aux bivalves (Exogyra conica, Costagyra olisiponensis,
Ceratostreon flabellatum, Rhynchostreon suborbiculatum, Plicatula auressensis, Eoradiolites liratus, Tenea delettrei), aux gastéropodes (Cimolithium tenouklense, Pterodonta deffisi, Nerinea texana) et aux échinides (Hemiaster gabrielis). La morphologie externe de ce polypier et son abondance indiquent un environnement peu profond, de faible énergie, à taux de sédimentation élevé et à substrat vaseux (Gill \& Chikhi, 1991). Les derniers termes de cette unité sont plus calcaires. Ils présentent un banc calcaire d'environ $4 \mathrm{~m}$ d'épaisseur, dont la limite supérieure est marquée par une surface durcie.

\section{Unité C: Marnes à Neolobites vibrayeanus}

Elle est composée essentiellement de marnes grisâtres sur plus de $70 \mathrm{~m}$ d'épaisseur. À la base, ces marnes friables livrent des rudistes (Sphaerulites depressus, Eoradiolites liratus, Radiolites sauvagesi) et d'autres bivalves (Granocardium carolinum, G. desvauxi, G. productum). Dans leur partie médiane, elles comportent un banc calcaire métrique, de couleur jaunâtre, bréchifié à la base et stratifié au sommet. Ce calcaire est armé d'un biostrome de rudistes. Il est de texture packstone, 


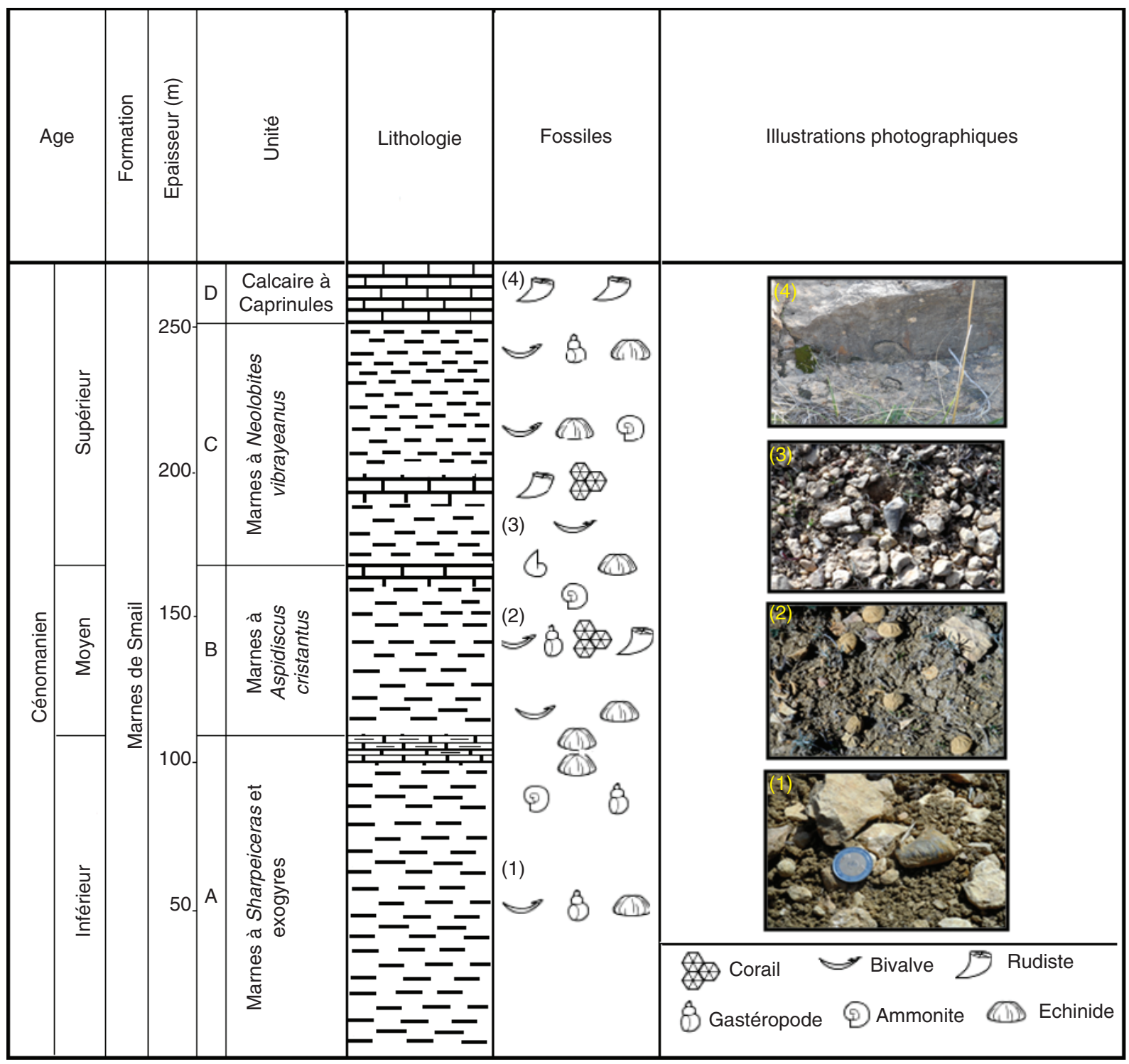

Fig. 3.-Coupe lithostratigraphique de Metrassi.

à milioles et cunéolines, et montre des traces d'oxydation. Les marnes sommitales livrent une faune assez diversifiée, avec des bivalves (Exogyra conica, Costagyra olisiponensis, Ceratostreon flabellatum, Ilymatogyra africana, Rhynchostreon suborbiculatum, Rastellum carinatum, Neithea dutrugei, Clisocolus corrugatus, Venericardia forgemoli, Granocarduim carolinum, G. desvauxi, Tenea delettrei), des gastéropodes (Campanile ganesha, Cimolithium tenouklense, Ampullina abeihensis, Columbellina fusiformis, Tylostoma pallaryi), des ammonites (Neolobites vibrayeanus), et des échinides (Heterodiadema libycum, Coenholectypus excisus, Hemiaster gabrielis). Cette unité témoigne d'une transgression sur un milieu marin peu profond.

\section{Unité D: Calcaire à Caprinules}

Cette unité se compose d'une barre calcaire à rudistes de $20 \mathrm{~m}$ d'épaisseur, marquant ainsi les derniers termes du Cénomanien supérieur. Les rudistes sont le plus souvent en bon état de conservation et d'une taille moyenne de $10 \mathrm{~cm}$. Ils sont attribués au genre Caprinula. Certains spécimens laissent distinguer 
une petite cavité accessoire postérieure, permettant leur attribution à Caprinula boissyi. Ces dépôts traduisent un milieu de plate-forme, des eaux chaudes, moins profondes et modérément agitées (ChickiAouimeur, 1995; Buchbinder et al., 2000; Benyoucef et al., 2012).

\section{Matériel étudié}

327 spécimens furent récoltés, par les auteurs, dans la succession cénomanienne de Djebel Metrassi. Ces spécimens ont été nettoyés, décrits et mesurés afin de clarifier leurs identification et attribution taxonomiques. Les abréviations des mesures suivantes ont été adoptées: H: hauteur; L: longueur; D: diamètre maximal; HL: hauteur de la dernière spirale; HA: hauteur de l'ouverture; WA: largeur de l'ouverture; PA : angle pleural en degrés; Nw: nombre de spirales.

\section{Biostratigraphie}

La distribution stratigraphique des macroinvertébrés identifiés a permis d'affiner le cadre biostratigraphique de la Formation des Marnes de Smail (Tableau 1):

- La présence de Sharpeiceras laticlavium, associée à Ceratostreon flabellatum et Rhynchostreon suborbiculatum, dans la partie inférieure de la formation (Unité A) lui attribue un âge Cénomanien inférieur. Cette unité correspondrait au niveau à Mantelliceras mantelli standard (Robaszynski et al., 1993) notamment celui d'Égypte (Abdel-Gawad et al., 2007).
- L'occurrence d'Aspidiscus cristatus, associée à Acanthoceras rhotomagense, attribue la partie moyenne de la même formation (Unité B) au Cénomanien moyen. Il s'agit de la même zone à Ceratostreon flabellatum et de la zone à Rhynchostreon mermeti (Abdel-Gawad et al., 2004a, b), ainsi que la zone à Hemiaster cubicus (Kora et al.,2001; Abdel-Gawad et al., 2007) en Égypte.

- La découverte de Neolobites vibrayeanus dans l'unité C de la Formation des Marnes de Smail permet de lui assigner la base du Cénomanien supérieur. Le niveau pré-Neolobites est marqué par la présence de Radiolites sauvagesi. Cette unité serait l'équivalent de la zone d'ammonite standard à Calycoceras guerangeri (Robaszynski et al., 1993). Elle l'est aussi pour la zone à Costagyra olisiponensis et Ilymatogyra africana (Abdel-Gawad et al., 2007) et celle à Costagyra olisiponensis (Ayoub-Hanna, 2011) en Égypte.

- La dernière unité (D) de la Formation des Marnes de Smail coïncide avec la barre calcaire à Caprinula boissyi. Ce rudiste permet de lui attribuer un âge Cénomanien supérieur, comparable aux niveaux à caprinules de la Formation des «Calcaires de Sidi Mohamed Ben Bouziane» (Benyoucef et al., 2012). Elle est aussi comparable aux sites équivalents de la Téthys occidentale (Chikhi-Aouimeur et al., 2006). Cette unité correspondrait à la zone d'ammonite à Metoicoceras geslinianum (Robaszynski et al., 1993).

Tableau 1.-Découpage biostratigraphique des dépôts de Metrassi.

\begin{tabular}{|c|c|c|c|c|c|c|c|c|c|c|c|c|c|}
\hline \multicolumn{2}{|c|}{ Age } & Zones d'ammonite & Autres fossiles & \multicolumn{10}{|c|}{ Range } \\
\hline \multirow{5}{*}{ 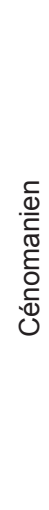 } & & & Caprinula boissyi & \multirow{5}{*}{ 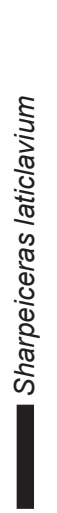 } & \multirow{5}{*}{ 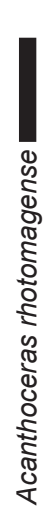 } & \multirow{5}{*}{\multicolumn{2}{|c|}{ 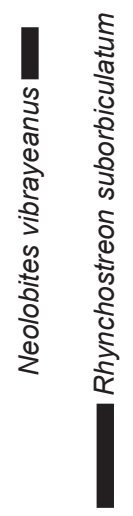 }} & \multirow{5}{*}{ 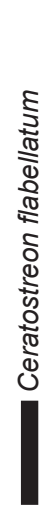 } & \multirow{5}{*}{ 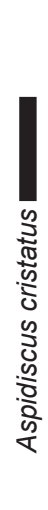 } & \multirow{5}{*}{ 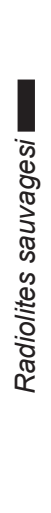 } & \multirow{5}{*}{ 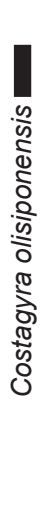 } & \multirow{5}{*}{ 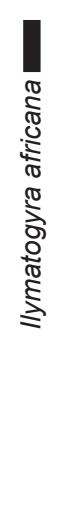 } & \multirow{5}{*}{ 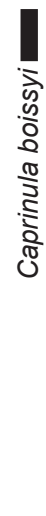 } \\
\hline & : & $\begin{array}{l}\text { Neolobites } \\
\text { vibrayeanus }\end{array}$ & $\begin{array}{l}\text { Costagyra olisiponensis } \\
\text { Ilymatogyra africana }\end{array}$ & & & & & & & & & & \\
\hline & & & Radiolites sauvagesi & & & & & & & & & & \\
\hline & 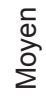 & $\begin{array}{l}\text { Acanthoceras } \\
\text { rhotomagense }\end{array}$ & $\begin{array}{l}\text { Aspidiscus } \\
\text { cristatus }\end{array}$ & & & & & & & & & & \\
\hline & 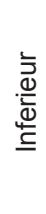 & $\begin{array}{l}\text { Sharpeiceras } \\
\text { laticlavium }\end{array}$ & $\begin{array}{c}\text { Rhynchostreon } \\
\text { suborbiculatum } \\
\text { Ceratostreon } \\
\text { flabellatum }\end{array}$ & & & & & & & & & & \\
\hline
\end{tabular}




\section{Systématique}

Corail

Ordre: Microsolenina MORYCOWA\& RONIEWICZ, 1995

Famille: Latomeandridae ALLOITEAU, 1952

Genre: Aspidiscus KÖNIG, 1825

Espèce: Aspidiscus cristatus (LAMARCK, 1801)

Fig. 4.1

1801 Cyclolites cristata sp. nov. LAMARCK, p. 369. 1914 Aspidiscus cristatus LAMARCK - FELIX, p. 107. (liste synonymique).

1930 Aspidiscus cristatus LAMARCK - RENZ, p. 8, pl. 2 , fig. 1.

1952 Aspidiscus cristatus LAMARCK ALLOITEAU, p. 663, pl. 9, fig. 12.

1957 Aspidiscus cristatus LAMARCK - THOMAS \& OMARA, p. 152, pls. 4-5.

1987 Aspidiscus cristatus LAMARCK - GILL \& LAFUSTE, p. 926, pl. 1, figs. 1-9 (non. fig. 10), 11-14; pl. 2, figs. 1-10; text-figs. 1-3a, 5.

1991 Aspidiscus cristatus LAMARCK - GILL \& CHIKHI, p. 349, figs. 1-2.

2002 Aspidiscus cristatus LAMARCK - BARONSZABO, p. 144, text-fig. 53A.

2011 Aspidiscus cristatus LAMARCK - AYOUBHANNAA et al., p. 294, text-fig. 5.8. J.

2011 Aspidiscus cristatus LAMARCK - PANDEY et al., p. 30, pl. 1, figs. 1-6.

Description: Polypier à surfaces inférieure concave et supérieure convexe, de contour circulaire hydnophoroïde. Couronne périphérique distincte, couverte de septes qui s'étendent jusqu'à la marge en angle droit. Ils continuent sous forme de côtes étroites au-dessus de la surface inférieure.

Discussion: Le rapport crête-monticule est souvent utilisé comme caractère discriminant entre les différentes espèces du genre Aspidiscus. Aspidiscus cristatus (LAMARCK) est dominé par des crêtes dont la médiane, plus longue, divise le dôme central à parts égales, avec quelques monticules. Par conséquent, Aspidiscus felixi (RENZ, 1930) est dépourvu de crêtes et présente des monticules emballés en masse. Chez Aspidiscus franchii (ZUFFARDICOMERCI, 1921), quelques crêtes courtes et plusieurs monticules dispersés sont moins nombreux que chez Aspidiscus montgrinensis (SOLÉ, 1937).
Répartition: Cénomanien moyen à supérieur: Espagne (Baron-Szabo, 2002), Algérie (Coquand, 1862; Gill \& Chikhi, 1991), Tunisie (Alloiteau, 1952), Égypte (Thomas \& Omara, 1957; Ayoub-Hannaa, 2011; Pandey et al., 2011).

Matériel: 20 spécimens collectés dans l'unité B.

\section{Bivalves}

La classification systématique utilisée est celle établie par Amler et al. (2000). L et H représentent respectivement les mesures des largeur et hauteur des coquilles, déterminées en $(\mathrm{mm})$.

Ordre: Nuculoida DALL, 1889

Famille: Nuculidae GRAY, 1824

Genre: Nucula LAMARCK, 1799

Espèce: Nucula margaritifera (DOUVILLÉ, 1916) Fig. 4.2.

1916 Nucula margaritifera sp. nov. DOUVILLÉ, p. 177 , pl. 21, figs. 19-21.

1963 Nucula awadensis sp. nov. FAWZI, p. 19, pl. 1, figs. 8-10.

2002 Nucula (Nucula) margaritifera DOUVILLÉ ABDEL-GAWAD \& GAMEIL, p. 77, pl. 1, fig. 1. 2006 Nucula (Nucula) margaritifera DOUVILLÉ EL QOT, p. 16, pl. 1, figs. 1-2, 5-6.

2007b Nucula (Nucula) margaritifera DOUVILLÉMEKAWY, p. 201, pl. 1, fig. 1.

2008 Nucula (Nucula) margaritifera DOUVILLÉ MEKAWY \& ABU-ZIED, p. 294, pl. 1, fig. 1. 2011 Nucula margaritifera DOUVILLÉ - AYOUBHANNAA, p. 51, pl. 3, fig. 1.

2014 Nucula margaritifera DOUVILLÉ - AYOUBHANNAA \& FÜRSICH, p. 67, pl. 1, fig. 1.

Description: Coquille de taille moyenne, ovale à allongée, légèrement gonflée et inéquilatérale. Crochet important situé au tiers de la longueur depuis l'extrémité postérieure. Ornementation absente.

Répartition: Cénomanien: Égypte (Fawzi, 1963; Mekawy, 2007b; Mekawy et Abu-Zied, 2008; AyoubHannaa, 2011; Ayoub-Hannaa \& Fürsich, 2014).

Matériel: 1 spécimen collecté dans l'unité A. Mesures:

\begin{tabular}{|l|c|c|c|}
\hline $\mathrm{n}=1$ & $\mathrm{H}$ & $\mathrm{L}$ & $\mathrm{H} / \mathrm{L}$ \\
\hline Spécimen & 14 & 24.72 & 0.56 \\
\hline
\end{tabular}




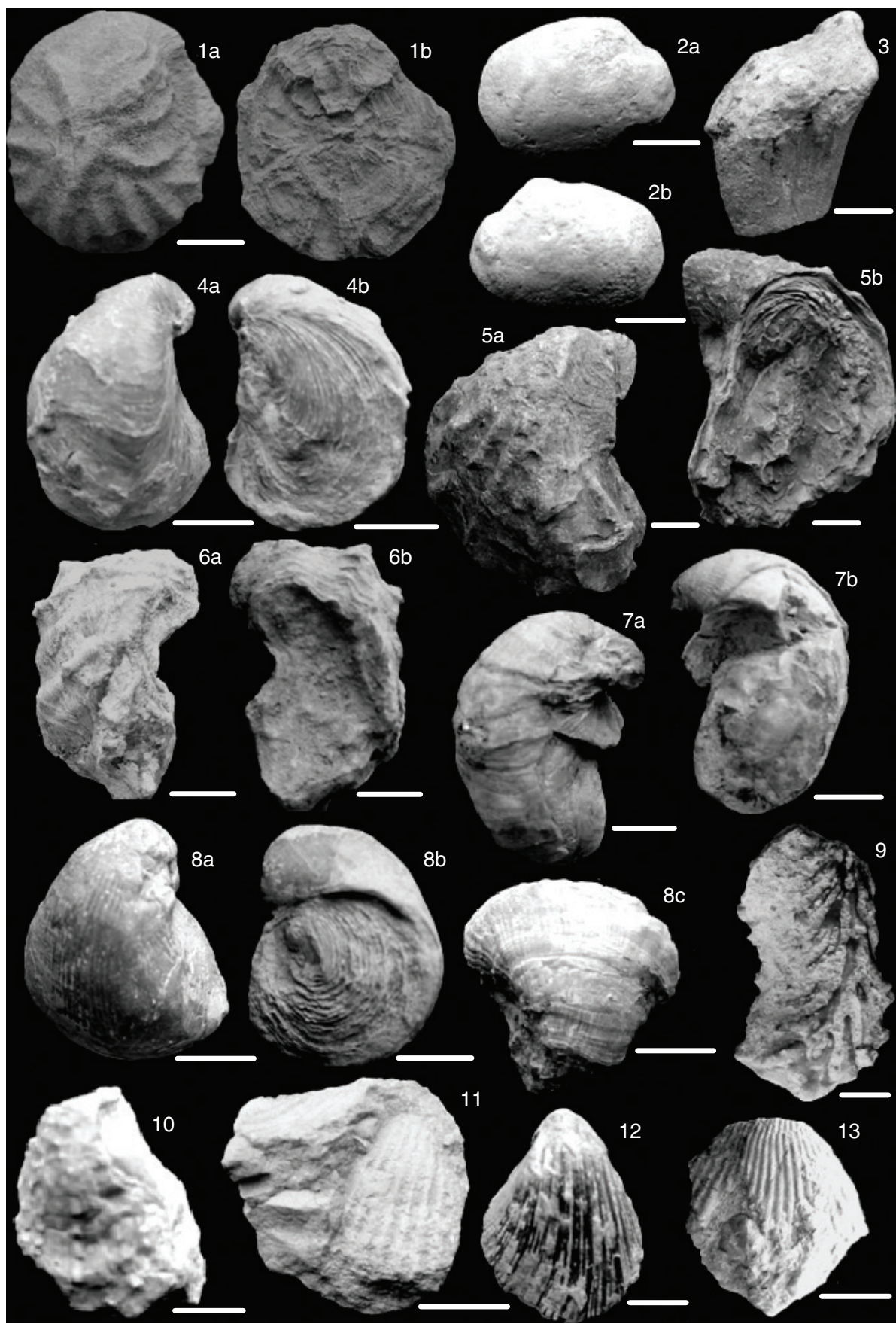

Fig. 4.-1. Aspidiscus cristatus (LAMARCK, 1801) de l'unité B: 1a- face supérieure, 1b- face inférieure. 2. Nucula margaritifera (DOUVILLÉ, 1916) de l'unité A: 2a- Valve gauche, 2b- valve droite. 3. Pinna sp. de l'unité A. 4. Exogyra conica (SOWERBY, 1813) de l'unité A. 4a- valve gauche, 4b-Valve droite. 5. Costagyra olisiponensis (SHARPE, 1850) de l'unité A: 5a- valve gauche, 5b- valve droite. 6. Ceratostreon flabellatum (GOLDFUSS, 1833) de l'unité A: 6a- valve gauche, 6b- valve droite. 7. Ilymatogyra africana (LAMARCK, 1801) de l'unité C: 7a- valve gauche, 7b- valve droite. 8. Rhynchostreon suborbiculatum (LAMARCK, 1801) de l'unité A: $8 \mathrm{a}-$ valve gauche, 8b- valve droite, 8c- vue frontale. 9. Rastellum carinatum (LAMARCK, 1806) de l'unité C. 10. Plicatula auressensis (COQUAND, 1862) de l'unité A. 11. Plicatula sp. de l'unité A. 12. Neithea coquandi (PÉRON, 1877) de l'unité A. 13. Neithea dutrugei (COQUAND, 1862) de l'unité C. (chaque barre correspond à $1 \mathrm{~cm}$ ). 
Ordre: Pterioida NEWELL, 1965

Famille: Pinnidae LEACH, 1819

Genre: Pinna LINNÉ, 1758

Espèce: Pinna sp.

Fig. 4.3.

Description: Spécimen équivalve, fortement inéquilatéral. Les deux valves sont peu convexes. Ornementation absente.

Matériel: 2 spécimens trouvés dans l'unité A.

Ordre: Ostreoida FERUSSAC, 1822

Famille: Gryphaeidae VYALOV, 1936

Genre: Exogyra SAY, 1820

Espèce: Exogyra conica (SOWERBY, 1813)

Fig. 4.4.

1813 Chama conica SOWERBY, p. 91, p. 69, pl. 26, fig. 3.

1833 Exogyra subcarinata GOLDFUSS, p. 44, vol. 2, p. 37, pl. 87, fig. 4.

1847 Ostrea conica D’ORBIGNY, p. 20, p. 720, pl. 478, figs. 5-8.

1869 Ostrea conica SOWERBY - COQUAND, pl. 53, figs. 1-7.

1912 Exogyra conica SOWERBY -

PERVINQUIÈRE, p. 182.

1912 Exogyra conica SOWERBY - WOODS, p. 407, text-figs. 215-242.

1917 Ostrea conica SOWERBY - FOURTAU, p. 32. 1934 Exogyra conica SOWERBY -

BLANCKENHORN, p. 202, pl. 9, fig. 44.1.

1959 Exogyra cf. conica SOWERBY -

BOREHAM, p. 121, pl. 11, figs. 1-3.

1972 Amphidonte conica SOWERBY - FRENEIX,

p. 85 , pl. 5, figs. 1a-b, 2a-b.

1981 Amphidonte conicum SOWERBY - AMARD

et al., p. 83, pl. 4, figs. 12-13.

1982 Amphidonte obliquatum SOWERBY -

DHONDT, p. 856.

2002 Exogyra conica SOWERBY - ABDEL-

GAWAD \& GAMEIL, p. 85, pl. 2, figs. 3-4.

2011 Exogyra conica SOWERBY-AYOUB-

HANNAA, p.76, pl. 5, figs.7-8; text-figs. 3-4.

2014 Exogyra conica SOWERBY - AYOUB-

HANNAA \& FÜRSICH, p. 78, pl. 3, figs. 4-6.

Description: Coquille de forme variable au plus jeune âge, mais régulière à l'âge adulte. Coquille inéquilatérale, souvent très convexe, plus ou moins sub-triangulaire ou semi-ovale. Valve gauche très convexe et valve droite mince. Operculiforme, souvent un peu concave, avec des lignes d'accroissement irrégulières. Crochets peu postérieurement incurvés en spirale.

Répartition: Albien-Cénomanien: Angleterre (Woods, 1912), Espagne (Dhondt, 1982) et NouvelleZélande (Boreham, 1959). Cénomanien: Maroc (Freineix, 1972), Algérie (Coquand, 1862; Amard et al., 1981), Tunisie (Pervinquière, 1912), Égypte (Fourtau, 1917; Ayoub-Hannaa, 2011; Ayoub-Hannaa \& Fürsich, 2014), Syrie et Palestine (Blanckenhorn, 1934).

Discussion: Selon Woods (1912), Exogyra haliotoidea est une petite forme de Exogyra conica. Rhynchostreon suborbiculatum (Lamarck, 1819) ressemble à Exogyra conica mais en diffère par une forme plus arrondie.

Matériel: 37 spécimens dont 31 récoltés dans l'unité A, 2 dans l'unité B et 4 dans l'unité C.

Mesures:

\begin{tabular}{|l|c|c|c|}
\hline $\mathrm{n}=37$ & $\mathrm{H}$ & $\mathrm{L}$ & $\mathrm{H} / \mathrm{L}$ \\
\hline Étendue & $20-44.60$ & $11.20-25.10$ & $1.28-1.79$ \\
\hline Moyenne & $30.12-32.66$ & $20.67-21.13$ & $1.42-1.58$ \\
\hline
\end{tabular}

Genre: Costagyra VYALOV, 1936

Espèce: Costagyra olisiponensis (SHARPE, 1850)

Fig. 4.5.

1850 Exogyra olisiponensis sp. nov. SHARPE, p. 185 , pl. 19, figs. 1-2.

1869 Ostrea olisoponensis COQUAND, p. 125, pl. 45, figs. 1-7.

1891 Ostrea olisiponensis SHARPE - PÉRON, pl. 23, figs. 14-18.

1912 Exogyra olisiponensis SHARPE -

PERVINQUIÈRE, p. 174, pl. 13, figs. 4-5, 9.

1918 Exogyra Olisiponensis SHARPE - GRECO, p. 187, pl. 1 (17), figs. 12-14.

1958 Exogyra olisiponensis SHARPE - BARBER, p. 21, pl. 8, figs. 3-4.

1972 Exogyra olisiponensis SHARPE - FRENEIX, p. 89 , pl. 5 , fig. 6 .

1990 Exogyra (Costagyra) olisiponensis SHARPE MALCHUS, p. 134, pl. 10, figs. 1-6.

1999 Exogyra (Costagyra) olisiponensis SHARPE SEELING \& BENGTSON, p. 756, fig. 9a-c. 2004 Costagyra olisiponensis SHARPE - ABDELGAWAD et al., pl. 7, fig. 1. 
2006 Exogyra (Costagyra) olisiponensis SHARPE PERRILLIAT et al., p. 99, figs. 10-11.

2011 Costagyra olisiponensis SHARPE - AYOUBHANNAA, p. 78, pl. 5, fig. 9; pl. 6, figs. 1-2; textfig. 3.5.

2014 Costagyra olisiponensis SHARPE - AYOUBHANNAA \& FÜRSICH, p. 81, pl., 3, fig. 1, pl. 4, figs. 1-2.

Description: Coquille de taille moyenne, inéquivalve, inéquilatérale et très épaisse. Elle présente différentes formes: arrondie, ovale ou exogyriforme. Valve gauche fortement convexe et très épaisse, ornée de nervures radiales pointues. Ces nervures sont croisées par des lamelles de croissance écailleuse. La valve droite est ornée de lamelles de croissance concentriques, dont une partie est traversée par des nervures radiales courtes. Crochet fortement incurvé.

Discussion: La grande variation entre les individus de Costagyra olisiponensis (Sharpe) a poussé plusieurs auteurs à proposer de nouveaux noms spécifiques tels que Exogyra oxyntax (Seguenza, 1882).

Répartition: Albien-Turonien: Brésil (Seeling \& Bengtson, 1999), Maroc (Freineix, 1972), Tunisie (Péron, 1891; Pervinquière, 1912), Égypte (Greco, 1918; Ayoub-Hannaa, 2011; Ayoub-Hannaa \& Fürsich, 2014), Palestine (Larlet, 1873), Jordanie (Perrilliat et al., 2006). Turonien: Nigeria (Barber, 1958) et Algérie (Coquand, 1862; Hardouin, 1868; Amard et al., 1981; Benyoucef et al., 2012).

Matériel: 10 spécimens dont 2 récoltés dans l'unité A, 4 dans l'unité B et 4 dans l'unité $C$.

Mesures:

\begin{tabular}{|l|c|c|c|}
\hline $\mathrm{n}=10$ & $\mathrm{H}$ & $\mathrm{L}$ & $\mathrm{H} / \mathrm{L}$ \\
\hline Étendue & $41.71-101.21$ & $30.52-102.60$ & $0.63-1.79$ \\
\hline Moyenne & $69.81-82.53$ & $60.71-70.64$ & $1.20-1.35$ \\
\hline
\end{tabular}

Genre: Ceratostreon BAYLE, 1878

Espèce: Ceratostreon flabellatum

(GOLDFUSS, 1833) Fig. 4.6.

1833 Exogyra flabellata sp. nov. GOLDFUSS, p. 38 , pl. 87 , fig. 6 .

1862 Ostrea flabellata COQUAND, p. 295.

1869 Ostrea flabellata COQUAND, p. 126, pl. 49.

figs. 1-2; pl. 52, figs. 1-9.

1912 Exogyra flabellata GOLDFUSS -

PERVINQUIËRE, p. 189, pl. 13, fig. 6a-b.
1937 Exogyra flabellata GOLDFUSS - TREVISAN, p. 37, pl. 5, figs. 11-12.

1972 Ceratostreon flabellatum GOLDFUSS -

FRENEIX, p. 91, pl. 5, figs. 8-9.

1990 Amphidonte (Ceratostreon) flabellatum

GOLDFUSS - MALCHUS, p. 111, pl. 4, figs. 4-11;

pl. 5, figs. 1-7. (avec synonymie).

1999 Amphidonte (Ceratostreon) flabellatum

GOLDFUSS - SEELING \& BENGTSON, p. 755 , fig. $8 \mathrm{a}-\mathrm{d}$.

2002 Amphidonte (Ceratostreon) flabellatum

GOLDFUSS - AHMAD \& EL-HAMMAD,

p. 450 , figs. $2 / 1,3,4,6$.

2002 Amphidonte (Ceratostreon) flabellatum

GOLDFUSS - BERNDT, p. 109, pl. 3, fig. 8a-b.

2002 Ceratostreon flabellatum GOLDFUSS -

ABDEL-GAWAD \& GAMEIL, p. 86, pl. 2, fig. 8.

2002 Ceratostreon flabellatum GOLDFUSS -

ABDELHAMID \& EL QOT, p. 269, pl. 3, fig. 2.

2002 Ceratostreon flabellatum GOLDFUSS -

KASSAB \& ZAKHERA, p. 8, fig. 3/13-15.

2004 Amphidonte (Ceratostreon) flabellatum

GOLDFUSS - KHALIL \& MASHALY, pl. 2, fig. 7.

2004a Ceratostreon flabellatum GOLDFUSS -

ABDEL-GAWAD et al., pl. 7, figs. 8, 9a- b.

2004b Amphidonte (Ceratostreon) flabellatum

GOLDFUSS) - ABDEL-GAWAD et al., pl. 2,

fig. 2a-b.

2006 Ceratostreon flabellatum GOLDFUSS - EL

QOT, p. 40, pl. 6, figs. 5-8; text-fig. 8.

2007b Ceratostreon flabellatum GOLDFUSS -

MEKAWY, p. 215, pl. 2, fig. 11; pl. 3, fig. 6 .

2008 Ceratostreon flabellatum GOLDFUSS -

MEKAWY \& ABU-ZIED, p. 301, pl. 2, fig. 1.

2011 Ceratostreon flabellatum GOLDFUSS -

AYOUB-HANNAA, p. 82, pl. 6, figs. 3-5; pl. 7,

figs.1-3; text-fig. 3.6.

2014 Ceratostreon flabellatum GOLDFUSS -

AYOUB-HANNAA \& FÜRSICH, p. 82, pl., 4,

figs. 3-5; pl. 5, figs. 1-3.

Description: Coquille exogyforme, ovale à oblique, plus haute que longue et inéquivalve. Elle montre un contour en croissant ou arrondi. La valve gauche est plus convexe et plus large que la valve droite. Crochet tordu et incurvé postérieurement. L'ornementation est composée occasionnellement de nervures en "V" inversé, en section transversale 
depuis la quille moyenne qui divise la coquille en deux parties de pente différente.

Répartition: Albien-Sénonien: Brésil (Seeling \& Bengston, 1999), Maroc (Freneix, 1972), Tunisie (Pervinquière, 1912), Égypte (Malchus, 1990; Ayoub-Hannaa, 2001; Ayoub-Hannaa \& Fürsich, 2014) et Jordanie (Ahmad \& El-Hammad, 2002; Berndt, 2002). Cénomanien: Algérie (Coquand, 1862; Amard et al., 1981; Benyoucef et al., 2012) et Italie (Trevisan, 1937; Russo, 1958).

Matériel: 13 spécimens dont 2 récoltés dans l'unité A, 1 dans l'unité B et 10 dans l'unité C.

\section{Mesures:}

\begin{tabular}{|l|c|c|c|}
\hline $\mathrm{n}=13$ & $\mathrm{H}$ & $\mathrm{L}$ & $\mathrm{H} / \mathrm{L}$ \\
\hline Étendue & $21.25-40.48$ & $10.05-32.64$ & $1.24-2.10$ \\
\hline Moyenne & $32.99-35.19$ & $18.54-19.73$ & $53.96-1.80$ \\
\hline
\end{tabular}

Genre: Ilymatogyra STENZEL, 1971

Espèce: Ilymatogyra africana (LAMARCK, 1801) Fig. 4.7.

1801 Gryphaea africana sp. nov. LAMARCK, p. 399, pl. 139, figs. 5-6.

1862 Ostrea auressensis sp. nov. COQUAND, p. 233, pl. 22, figs. 12-13.

1912 Exogyra Africana LAMARCK -

PERVINQUIÈRE, p. 184.

1937 Exogyra africana LAMARCK - TREVISAN, p. 72, pl. 3, fig. 18; pl. 5, figs. 1-4.

1962 Exogyra africana LAMARCK - ABBASS, p. 65 , pl. 9 , figs. $2-3$.

1999 Ilymatogyra (Afrogyra) africana LAMARCK SEELING \& BENGTSON, p. 758, fig. 9d-g.

2002 Ilymatogyra (Afrogyra) africana LAMARCK BERNDT, p. 110, pl. 3, figs. 5-7 (forma typica and forma crassa).

2002 Ilymatogyra (Afrogyra) africana LAMARCKAHMAD \& AL-HAMMAD, p. 452, figs. 2/7-10, non fig. 2.

2004 Ilymatogyra (Afrogyra) africana LAMARCK KHALIL \& MASHALY, pl. 2, figs. 1-2.

2004a Ilymatogyra africana LAMARCK - ABDELGAWAD et al., pl. 7, figs. 2-3.

2004b Ilymatogyra africana LAMARCK - ABDELGAWAD et al., pl. 1, fig. 15.

2006 Ilymatogyra africana LAMARCK - EL QOT, p. 42 , pl. 7, figs. $1-7,8 \mathrm{a}-\mathrm{b}$; text-fig. $7 \mathrm{~b}$.

2007 Ilymatogyra africana LAMARCK - ABDELGAWAD et al., pl. 5, fig. 8 . 2007b Ilymatogyra africana LAMARCK MEKAWY, p. 216, pl. 3, fig. 2.

2011 Ilymatogyra africana LAMARCK - AYOUBHANNAA, p. 82, pl. 7, figs. 4-6; text-fig. 3.7. 2014 Ilymatogyra africana LAMARCK - AYOUBHANNAA \& FÜRSICH, p. 84, pl., 5, figs. 4-6.

Description: Coquille de taille moyenne, allongée, plus haute que longue, inéquivalve et inéquilatérale. Valve gauche plus convexe que la valve droite. Crochet petit et peu tordu. La valve gauche est ornée de lamelles écailleuses ou lisses, à croissance régulière.

Répartition: Cénomanien: Brésil (Seeling \& Bengtson, 1999), Algérie (Coquand, 1862; Benyoucef et al., 2012; Benyoucef et al., 2016), Tunisie (Pervinquière, 1912), Égypte (Abbass, 1962; AyoubHannaa, 2011; Ayoub-Hannaa \& Fürsich, 2014), Jordanie (Ahmad \& El-Hammad, 2002; Berndt, 2002) et Italie (Trevisan, 1937).

Matériel: 1 spécimen récolté dans l'unité C.

Mesures:

\begin{tabular}{|l|c|c|c|}
\hline $\mathrm{n}=1$ & $\mathrm{H}$ & $\mathrm{L}$ & $\mathrm{H} / \mathrm{L}$ \\
\hline Spécimen & 32.03 & 22.50 & 1.42 \\
\hline
\end{tabular}

Genre: Rhynchostreon BAYLE, 1878

\section{Espèce: Rhynchostreon suborbiculatum} (LAMARCK, 1801) Fig. 4.8.

1801 Gryphaea suborbiculata sp. nov. - LAMARCK, p. 398, pl. 23, figs. 11-13.

1819 Gryphaea columba sp. nov. LAMARCK, p. 198. 1862 Ostrea Mermeti sp. nov. COQUAND, p. 234, pl. 23, figs. 3-5.

1871 Exogyra suborbiculata LAMARCK STOLICZKA, p. 462, pl. 35, figs. 1-4.

1912 Exogyra columba LAMARCK PERVINQUIÈRE, p. 180.

1918 Exogyra columba LAMARCK - GRECO, p. 7 (189), pl. 1 (17), figs. 15-18; pl. 2 (18), figs. 1-4. 1972 Rhynchostreon columbum LAMARCK mermeti COQUAND - FRENEIX, p. 88, pl. 5, figs. 4, 5. 1982 Rhynchostreon suborbiculatum LAMARCK DHONDT, p. 61, fig. 3a.

2002 Rhynchostreon mermeti COQUAND - AHMAD \& ABDELHAMID, p. 456, figs. 3/4-11; figs. 4/1-2. 2004 Rhynchostreon suborbiculatum LAMARCK KHALIL \& MASHALY, pl. 2, figs. 3-4. 
2004a Rhynchostreon suborbiculatum LAMARCK ABDEL-GAWAD et al., pl. 7, figs. 4a-b, 6.

2004b Rhynchostreon mermeti COQUAND ABDEL-GAWAD et al., pl. 1, fig. 16. 2006 Rhynchostreon suborbiculatum LAMARCK EL QOT, p. 43, pl. 7, figs. 9a-11; pl. 8, figs. 1-4, text-fig. $8 \mathrm{~B}$.

2007b Rhynchostreon suborbiculatum LAMARCK MEKAWY, p. 217, pl. 3, figs. 3, 4.

2011 Rhynchostreon suborbiculatum LAMARCK AYOUB-HANNAA, p. 82, pl. 7, figs. 7-9;

text-fig. 3.8.

2014 Rhynchostreon suborbiculatum LAMARCK AYOUB-HANNAA \& FÜRSICH, p. 86, pl. 5, figs. 7-10.

Description: Coquille de petite à moyenne taille, plus haute que longue et inéquilatérale. Forme ovale, allongée à exogyriforme. La valve gauche est plus convexe et plus grande que la valve droite. Crochet petit et modérément tordu. L'ornementation se caractérise par la présence de lamelles de croissance marginales sur la valve droite et de nervures radiales sur la valve gauche.

Discussion: Ostrea columba de Coquand récoltée en Algérie est conspécifique à Rhynchostreon suborbiculatum et les différences mentionnées auparavant dans plusieurs travaux sont parfois inexistantes (Thomas, 1891).

Répartition: Cénomanien: France (Dhondt, 1985), Portugal (Soares, 1966; Choffat, 1901), Maroc (Freneix, 1972), Algérie (Coquand, 1862; Collignon, 1971; Benyoucef et al., 2012), Tunisie (Pervinquière, 1912), Égypte (Greco, 1918; Malchus, 1990; AyoubHannaa, 2011; Ayoub-Hannaa \& Fürsich, 2014), Jordanie (Aqrabawi, 1993; Ahmad \& El-Hammad, 2002) et l'Inde (Stoliczka, 1871).

Matériel: 42 spécimens dont 5 récoltés dans l'unité A, 3 dans l'unité B et 34 dans l'unité C.

\section{Mesures:}

\begin{tabular}{|l|c|c|c|}
\hline $\mathrm{n}=42$ & $\mathrm{H}$ & $\mathrm{L}$ & $\mathrm{H} / \mathrm{L}$ \\
\hline Étendue & $14.87-28.12$ & $8.49-39.61$ & $0.71-1.75$ \\
\hline Moyenne & $20.14-22.22$ & $18.43-20.06$ & $1.19-1.34$ \\
\hline
\end{tabular}

Famille: Ostreidae WILKES, 1810

Genre: Rastellum FAUJAS-SAINT-FOND, 1799

Espèce: Rastellum carinatum (LAMARCK, 1806)

Fig. 4.9.
1767 Ostrea diluviana LINNÉ, p. 1148.

1806 Ostrea carinata sp. nov. LAMARCK, p. 166. 1827 Ostrea diluviana, LINNÉ - NILSSON, p. 32, pl. 6, figs. la-c, 2.

1871 Ostrea (Alectryonia) carinata LAMARCK STOLICZKA, p. 468, pl. 48, fig. 5; pl. 49, figs. 1-2. 1894 Ostrea diluviana LINNÉ - LUNDGREN, p. 36. 1912 Ostrea diluviana, LINNÉ -WOODS, p. 342, text-figs. 98-138.

1938 Ostrea diluviana LINNÉ - CARLSSON, p. 10. 1982 Lopha diluviana LINNÉ - GRUNDEL, p. 157, pl. 4, figs. 6-10.

2004a Rastellum carinatum LAMARCK - ABDELGAWAD et al., pl. 8, figs. 9-10.

2004b Rastellum (Arctostrea) carinatum

LAMARCK - ABDEL-GAWAD et al., pl. 2, fig. 5. 2006 Rastellum carinatum (LAMARCK) - EL

QOT, p. 59, pl. 11, figs. 5, 6-7.

2011 Rastellum carinatum LAMARCK - AYOUBHANNAA, p. 100, pl. 9, figs. 1-2.

2014 Rastellum carinatum LAMARCK - AYOUBHANNAA \& FÜRSICH, p. 94, pl. 7, figs. 1-2.

Description: Coquille épaisse de taille moyenne, en forme de croissant. Flanc postérieur faiblement incliné. La valve gauche se divise en deux par une quille à partir de laquelle se développent des nervures bien marquées dans les deux sens vers la marge.

Répartition: Crétacé Supérieur: Inde (Stoliczka, 1871), Angleterre (Woods, 1912) et Égypte (Abdel Gawad et al., 2004a, 2004b; Ayoub-Hannaa, 2011; Ayoub-Hannaa \& Fürsich, 2014).

Matériel: 4 spécimens trouvés dans l'unité C.

Famille: Plicatulidae WATSON, 1930

Genre: Plicatula LAMARCK, 1801

Espèce: Plicatula auressensis (COQUAND, 1862)

Fig. 4.10.

1862 Plicatula auressensis sp. nov. COQUAND, p. 222, pl. 16, figs. 14-16.

1862 Plicatula Reynesi sp. nov COQUAND, p. 222, pl. 17, figs. 1-2.

1934 Plicatula reynesi COQUAND -

BLANCKENHORN, p. 193.

1958 Plicatula auressensis COQUAND -

BARBER, p. 20, pl. 7, fig. 4.

1962 Plicatula auressensis COQUAND -

ABBASS, p. 61, pl. 7, figs. 17-19. 
1981 Plicatula auressensis COQUAND - AMARD et al., p. 72.

2004b Plicatula reynesi COQUAND - ABDELGAWAD et al., pl. 1, fig. 13.

2006 Plicatula auressensis COQUAND - EL QOT, p. 62, pl. 12, figs. 2a-b, 3-4.

2011 Plicatula auressensis COQUAND - AYOUBHANNAA, p. 100, pl. 9, figs. 5-6.

2014 Plicatula auressensis COQUAND - AYOUBHANNAA \& FÜRSICH, p. 96, pl. 7, figs. 5-6.

Description: Coquilles incomplètes qui laissent apercevoir une forme ovale ou triangulaire. Ornementation composée de nervures radiales fortes au nombre de 4 qui se développent à une petite distance du crochet. Elles deviennent épineuses vers la marge ventrale, sur lesquelles se montrent des lamelles concentriques pas trop visibles.

Discussion: Selon Coquand, cette espèce rappelle, par sa forme générale, Plicatula Garteroni d'Orbigny. Elle s'en distingue par un nombre de côtes plus considérable et par l'absence de pointes droites sur les côtes principales.

Répartition: Cénomanien: Algérie (Coquand, 1862; Amard et al., 1981; Zaoui, 2017), Tunisie (Pervinquière, 1912), Égypte (Abbas, 1962; AyoubHannaa, 2011; Ayoub-Hannaa \& Fürsich, 2014), Italie (Trevisan, 1937) et Syrie (Blanckenhorn, 1934). Coniacien: Nigeria (Barber, 1958). Maestrichtien: Algérie (Collignon, 1971).

Matériel: 2 spécimens dont 1 récolté dans l'unité A et 1 dans l'unité $B$.

\section{Espèce: Plicatula sp.}

Fig. 4.11.

Description: Spécimen de petite taille qui n'apparaît pas dans sa totalité. Forme ovale à triangulaire, non épineuse, ornée de nervures marquées et assez espacées.

Matériel: 1 spécimen récolté dans l'unité A.

Ordre: Pectinoida NEWELL \& BOYD, 1995

(= Pectinina WALLER, 1978)

Famille: Pectinidae WILKES, 1810

Genre: Neithea DROUET, 1824

Espèce: Neithea coquandi (PÉRON, 1877)

Fig. 4.12.
1813 Neithea regularis SCHLOTHEIM, pl. 1, fig. 8; pI. 2, figs. 1, 4 .

1847 Janira quadricostata D'ORBIGNY, p. 644, pl. 447, figs. 1-7.

1850 Janira Geinitzii D’ORBIGNY, p. 197.

1862 Janira tricostata sp. nov. COQUAND, p. 219, pl. 13, figs. 3-4.

1912 Pecten (Neithea) Shawi sp. nov.

PERVINQUIÈRE, p. 136, pl. 9, figs. 1-6.

1934 Pecten (Vola) Shawi PERVINQUIÈRE -

BLANCKENHORN, p. 191, pl. 9, fig. 24.

1937 Pecten (Neithea) Shawi PERVINQUIÈRE -

TREVISAN, p. 55, pl. 3, fig. 2.

1973 Neithea (Neithea) regularis SCHLOTEIM -

DHONDT, p. 20, figs. 1, 3 .

2004b Neithea (Neithea) coquandi PÉRON -

ABDEL-GAWAD et al., pl. 1, fig. 11.

2011 Neithea (Neithea) coquandi PÉRON - AYOUB-

HANNAA, p.100, pl. 9, fig. 9; text-fig. 3.11.

2014 Neithea coquandi PÉRON - AYOUB-

HANNAA \& FÜRSICH, p. 98, pl. 7, fig. 10.

Description: Coquille moyenne à petite, ovale, inéquivalve et équilatérale. Valve droite fortement plus convexe que la valve gauche. Présence de trois nervures intercalées entre chaque couple de nervures principales (en nombre de cinq). Le bec est fortement incurvé vers l'intérieur.

Discussion: Neithea coquandi est facilement reconnaissable des autres espèces du genre Neithea en ayant trois nervures qui sont en grande partie inégales.

Répartition: Cénomanien: Tunisie (Pervinquière, 1912) et Égypte (Abdel Gawad et al., 2004b; AyoubHannaa, 2011; Ayoub-Hannaa \& Fürsich, 2014). Turonien: France (Dhondt, 1973) et Italie (Trevisan, 1937). Santonien: Algérie (Coquand, 1862, 1969) et Turquie (Dartevelle \& Brévion, 1956).

Matériel: 3 spécimens trouvés dans l'unité A.

Mesures:

\begin{tabular}{|l|c|c|c|}
\hline $\mathrm{n}=3$ & $\mathrm{H}$ & $\mathrm{L}$ & $\mathrm{H} / \mathrm{L}$ \\
\hline Étendue & $16.25-40.34$ & $12.31-31.27$ & $1.29-1.32$ \\
\hline Moyenne & $23.64-35.42$ & $21.70-21.81$ & $0.98-1.14$ \\
\hline
\end{tabular}

Espèce: Neithea dutrugei (COQUAND, 1862)

Fig. 4.13.

1862 Janira dutrugei sp. nov. COQUAND, p. 219 , pl. 13, figs. 1-2. 
1902 Vola dutrujei COQUAND var. beirensis CHOFFAT, p. 150, pl. 2, figs. 1-2. 1918 Pecten (Neithea) dutrugei COQUAND GRECO, p. 24 (206), pl. 3 (19), figs. 7-9. 1934 Pecten (Vola) dutrugei COQUAND BLANCKENHORN, p. 192, pl. 9, fig. 25. 1934 Pecten (Vola) zakarjensis sp. nov. BLANCKENHORN, p. 192, pl. 9, fig. 26. 2004b Neithea (Neithea) dutrugei COQUAND ABDEL-GAWAD et al., p. 323, pl. 1, fig. 12a-b. 2006 Neithea (Neithea) dutrugei COQUAND - EL QOT, p. 65, pl. 12, figs. 16-17. 2006 Neithea (Neithea) dutrugei COQUAND PERRILLIAT et al., p. 101, figs. 14-16. 2007b Neithea (Neithea) dutrugei COQUAND MEKAWY, p. 225, pl. 4, fig. 6.

2011 Neithea (Neithea) dutrugei COQUAND AYOUB-HANNAA, p. 100, pl. 9, fig. 10; text-fig. 3.12. 2014 Neithea dutrugei COQUAND - AYOUBHANNAA \& FÜRSICH, p. 98, pl. 7, fig. 11.

Description: Coquille de petite taille, à contour ovale, inéquivalve et légèrement inéquilatérale. Marge ventrale sub-circulaire et assez large. La valve gauche est ornée de plusieurs nervures principales et secondaires lisses disposées aléatoirement.

Discussion: Neithea dutrugei se distingue facilement des autres espèces du genre par la présence de nombreuses nervures irrégulières.

Répartition: Cénomanien: Portugal (Choffat, 1902), Égypte (Greco, 1918; Ayoub-Hannaa, 2011; Ayoub-Hannaa \& Fürsich, 2014), Syrie et Palestine (Blanckenhorn, 1934), Algérie (Coquand, 1862). Turonien inférieur: Jordanie (Perrilliat et al., 2006).

Matériel: 2 spécimens récoltés dans l'unité $\mathrm{C}$.

Ordre: Trigonioida DALL, 1889

Famille: Trigoniidae LAMARCK, 1819

Genre: Pterotrigonia VAN HOEPEN, 1929

Espèce: Pterotrigonia scabra (LAMARCK, 1819) Fig. 5.1

1819 Trigonia scabra sp. nov. LAMARCK, p. 63, no. 2.

1819 Trigonia crenulata sp. nov. LAMARCK, p. 63, no. 3 .

1871 Trigonia scabra LAMARCK - STOLICZKA, p. 314 , pl. 15 , figs. $24-26$; pl. 16 , figs. $35-40$.
1880 Lyriodon ethra sp. nov. COQUAND, p. 388. 1912 Trigonia Ethra COQUAND -

PERVINQUIÈRE, p. 218, pl. 15, figs. 4-7. 1917 Trigonia Ethra COQUAND - FOURTAU, p. 59.

1937 Trigonia Ethra COQUAND - TREVISAN, p. 81 , pl. 5, fig. 13a-b.

2002 Pterotrigonia (Scabrotrigonia) ethra COQUAND - ABDEL-GAWAD \& GAMEIL, p. 89 , pl. 2, fig. 14 .

2002 Pterotrigonia (Scabrotrigonia) sp. -

ABDELHAMID \& EL QOT, p. 273, pl. 4, fig. 4. 2002 Pterotrigonia (Scabrotrigonia) scabra LAMARCK - KORA et al., pl. 2, fig. 12. 2004b Trigonia ethra COQUAND - ABDELGAWAD et al., pl. 2, fig. 7.

2006 Pterotrigonia (Scabrotrigonia) scabra LAMARCK - EL-HEDENY, p. 711, pls. 1-2. 2006 Pterotrigonia (Scabrotrigonia) scabra LAMARCK - EL QOT, p. 65, pl. 13, figs. 3-5. 2007b Pterotrigonia (Scabrotrigonia) scabra LAMARCK - MEKAWY, p. 226, pl. 4, fig. 10. 2008 Pterotrigonia (Scabrotrigonia) scabra LAMARCK - MEKAWY \& ABU-ZIED, p. 307, pl. 2, fig. 13.

2011 Pterotrigonia (Scabrotrigonia) scabra LAMARCK - AYOUB-HANNAA, p. 112, pl. 10, fig. 2; text-fig. 3.15.

2014 Pterotrigonia (Scabrotrigonia) scabra LAMARCK - AYOUB-HANNAA \& FÜRSICH, p. 101, pl. 8, fig. 2 .

Description: Coquille de taille moyenne, aussi haute que longue, à contour en croissant, très inéquilatérale et équivalve. Crochet étroit et peu pointu. Ornementation se composant de lignes arquée, trés espacées, plus prononcées vers la marge postérieure. Elle se développe à partir de la quille médiane.

Répartition: Cénomanien-Santonien: Tunisie (Pervinquière, 1912), Égypte (Fourtau, 1917 AyoubHannaa, 2011; Ayoub-Hannaa \& Fürsich, 2014) et Italie (Trevisan, 1937). Santonien: Inde (Stoliczka, 1871).

Matériel: 1 spécimen trouvé dans l'unité A. Mesures:

\begin{tabular}{|l|c|c|c|}
\hline $\mathrm{n}=1$ & $\mathrm{H}$ & $\mathrm{L}$ & $\mathrm{H} / \mathrm{L}$ \\
\hline Spécimen & 31.72 & 26.65 & 1.19 \\
\hline
\end{tabular}




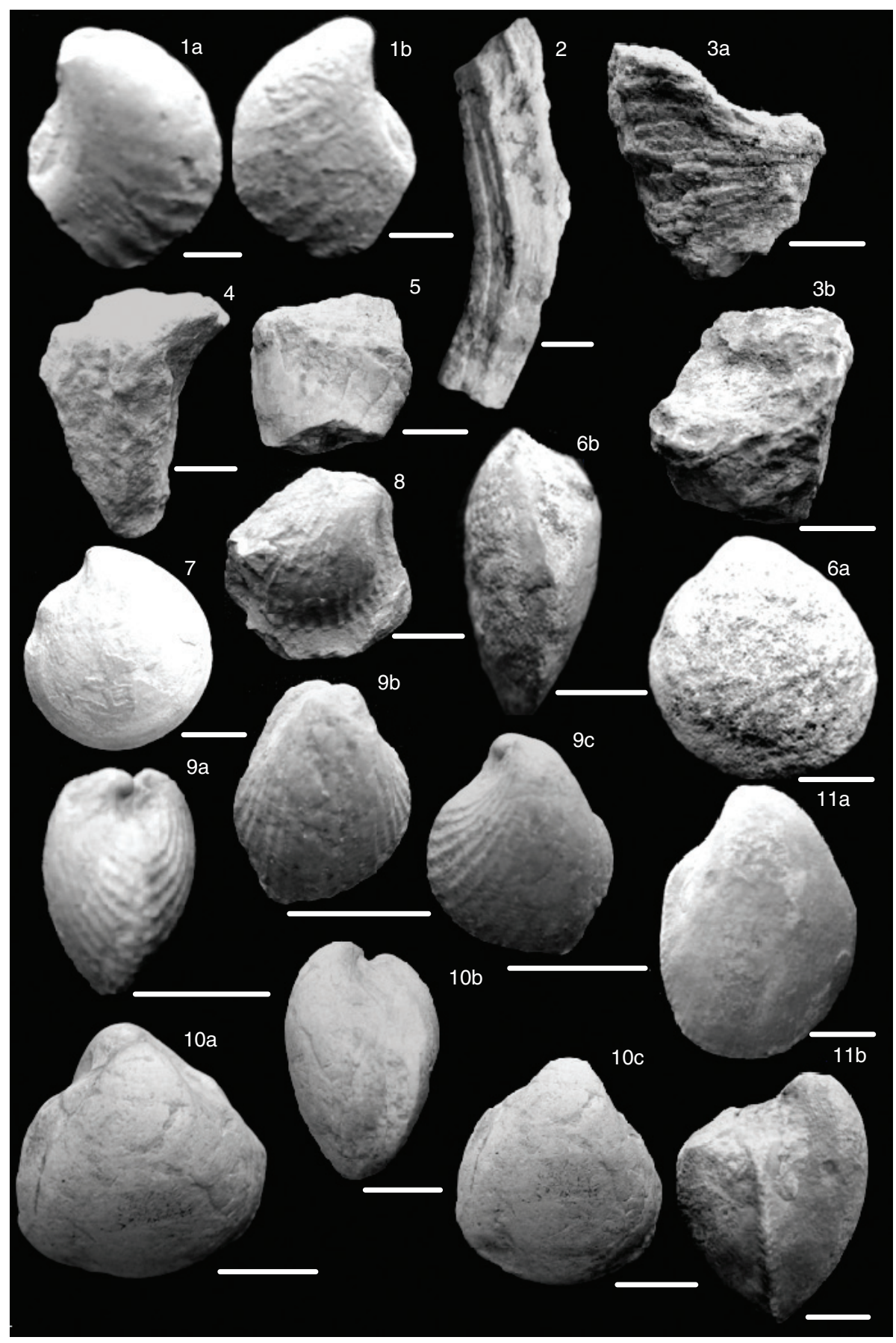

Fig. 5.-1. Pterotrigonia scabra (LAMARCK, 1819) de l'unité A: 1a- valve gauche, 1b- valve droite. 2. Caprinula boissyi (D'ORBIGNY, 1839) de l'unité D. 3. Radiolites sauvagesi (D'HOMBRES-FIRMAS, 1838) de l'unité C: 3a- valve droite, 3b- vue dorsale. 4 . Eoradiolites liratus (CONRAD, 1852) de l'unité C. 5. Sphaerulites depressus (BLANCKENHORN, 1934) de l'unité C. 6. Lucina fallax (FORBES, 1846) de l'unité C: 6a- valve droite, 6b- vue de coté. 7. Clisocolus corrugatus POPENOE, 1937 de l'unité C. 8. Venericardia forgemoli (COQUAND, 1862) de l'unité C. 9. Granocardium carolinum (D'ORBIGNY, 1843) de l'unité C: 9a- vue de coté, 9b- valve gauche, 9c- valve droite. 10. Granocardium desvauxi (COQUAND, 1862) de l'unité C: 10a-valve droite, 10b- vue de côté, 10c- valve gauche. 11. Granocardium (Granocardium) productum (J. DE C. SOWERBY, 1832) de l'unité C: 11a- valve droite, 11b- vue de coté. (chaque barre correspond à $1 \mathrm{~cm})$. 
Ordre: Hippuritoida NEWELL, 1965

Famille: Caprinidae D’ORBIGNY, 1850

Genre: Caprinula D'ORBIGNY, 1847

Espèce: Caprinula boissyi (D'ORBIGNY, 1840)

Fig. 5.2

1840 Caprina boissyi, D'ORBIGNY, p. 169.

1847 Caprinula boissyi D’ORBIGNY, pl. 7, fig. 52.

1888 Caprinula boissyi D’ORBIGNY -

DOUVILLÉ, p. 707, pl. 22, figs. $1 \mathrm{a}$, b.

2007 Caprinula boissyi D'ORBIGNY - TENTOR,

p. 6, figs. 4-5.

2010 Caprinula boissyi D'ORBIGNY - CHIKHIAOUIMEUR, p. 92, 93, figs. 83, 1-3, figs. 84, 1-4. 2016 Caprinula boissyi D'ORBIGNY - ÖZER \& AHMAD, p. 148, figs. 6E, 6F.

Description: Rudiste à valve gauche plus grande que la droite. Les moules externes incomplets montrent une ornementation composée de nervures longitudinales minces. La couche externe de la valve gauche laisse observer le canal caractéristique du genre Caprinula.

Discussion: Caprinula boissyi d'Orbigny et Caprinula cedrorum (Blanckenhorn) sont très semblables mais la première espèce se distingue par une cavité accessoire postérieure plus petite.

Répartition: Cénomanien supérieur: Algérie (Chikhi-Aouimeur, 1995, 2010; Benyoucef \& Meister, 2015; Benyoucef et al., 2016), Liban (Douvillé, 1910).

Matériel: 30 spécimens trouvés dans l'unité D.

Famille: Radiolitidae GRAY, 1848

Genre: Radiolites LAMARCK, 1801

Espèce: Radiolites sauvagesi (D'HOMBRESFIRMAS, 1838) Fig. 5.3.

1838 Sphaerulites sauvagesi $\mathrm{sp}$. nov. D'HOMBRES-FIRMAS, p. 193, pl. 2.

1999 Radiolites sauvagesi D'HOMBRES-FIRMAS STEUBER, p. 101, pl. 17, figs. 1-14; pl. 18, figs. 1-5; text-figs. 40-41.

2005 Radiolites sauvagesi D'HOMBRES-FIRMAS ALY et al., p. 263, pl. 6, figs. 4-5; pl. 7, fig. 1a-b. 2007 Radiolites sauvagesi D'HOMBRES-FIRMAS EL-HEDNEY, p. 91, fig. 8e-h.

2011 Radiolites sauvagesi D'HOMBRES-FIRMAS AYOUB-HANNAA, p. 112, pl. 10, figs. 7-8;

text-fig. 3.18.
2014 Radiolites sauvagesi D'HOMBRES-FIRMAS AYOUB-HANNAA \& FÜRSICH, p. 105, pl. 8, figs. 7-8.

Description: De forme cylindrique à section circulaire, de $50 \mathrm{~mm}$ de hauteur et $35 \mathrm{~mm}$ de diamètre. Ornementation composée de lamelles plicatives en pente moyenne.

Discussion: La structure interne de Radiolites sauvagesi ressemble étroitement à celle de Durania arnaudi (CHOFFAT, 1891) mais en diffère par une arête ligamentaire bien développée.

Répartition: Crétacé Supérieur: Égypte (Aly et al., 2005; Ayoub-Hannaa, 2011; Ayoub-Hannaa \& Fürsich, 2014). Cénomanien moyen à supérieur: Algérie (Chikhi-Aouimeur, 1998). Coniacien: Grèce (Steuber, 1999).

Matériel: 3 spécimens trouvés dans l'unité C.

Genre: Eoradiolites DOUVILLÉ, 1909

Espèce: Eoradiolites liratus (CONRAD, 1852)

Fig. 5.4.

1852 Hippurites liratus sp. nov. CONRAD, p. 234, pl. 7, figs. 47-48.

1913 Eoradiolities lyratus CONRAD - DOUVILLÈ, p. 244, pl. 14 (1), figs. 3-11; pl. 16 (3), fig. 8; text-fig. 6. 1998 Eoradiolities lyratus CONRAD - MASSE et al., p. 54, figs. 9/1-9.

2004a Eoradiolites liratus CONRAD - ABDELGAWAD et al., p. 293, pl. 9, figs. 8-9.

2004b Eoradiolites liratus CONRAD - ABDEL-

GAWAD et al., p. 372, pl. 4, fig. 2.

2005 Eoradiolites liratus CONRAD - SADOONI, p. 220 , text-fig. 5 .

2005 Eoradiolites liratus CONRAD - ALY et al., p. 256, pl. 1, figs. 4-8; pl. 2, fig. 2a-b.

2006 Eoradiolites liratus CONRAD - EL QOT, p. 69 , pl. 13, figs. 13-15.

2007 Eoradiolites liratus CONRAD - EL

HEDENY, p. 90, fig. 6/n-r.

2007b Eoradiolites liratus CONRAD - MEKAWY, p. 227, pl. 4, figs. 11, 12.

2008 Eoradiolites liratus CONRAD - MEKAWY

\& ABU-ZIED, p. 308, pl. 2, fig. 15.

2011 Eoradiolites liratus CONRAD - AYOUB-

HANNAA, p. 120, pl. 11, figs. 1-3; text-fig. 3.19.

2011 Eoradiolites liratus CONRAD - ZAKHERA, p. 419, pl. 2, figs. 1-4. 
2014 Eoradiolites liratus CONRAD - AYOUBHANNAA et al., p. 106, pl. 9, figs. 1-3.

Description: Spécimen de taille moyenne, de forme conique à section transverse sub-arrondie à sub-ovale. Des côtes radiales, courant sur toute la longueur de la valve, croisées par des lames de croissance.

Répartition: Cénomanien: Égypte (Douvillé, 1913; Ayoub-Hannaa, 2011; Ayoub-Hannaa et al., 2014), Italie (Masse et al., 1998) et Iraq (Sadoouni, 2005).

Matériel: 3 spécimens dont 1 trouvé dans l'unité B et 2 dans l'unité $C$.

Genre: Sphaerulites LAMARCK, 1819

Espèce: Sphaerulites depressus

(BLANCKENHORN, 1934) Fig. 5.5

1934 Sphaerulites depressus BLANCKENHORN, p. 229, pl. 12, figs. 104-106.

2011 Sphaerulites depressus BLANCKENHORN ZAKHERA, p. 423, pl. 4, figs. 3-9.

Description: Coquille large, en forme de vase, à cavité palléale relativement petite, ornée de nervures radiales très fines et espacées.

Discussion: Le genre Sphaerulites est caractérisé par des lames de croissance foliacées et des bandes radiales déprimées et flanquées de plis (Masse et al., 2007).

Matériel: 2 spécimens trouvés dans l'unité C.

Ordre: Veneroida H. ADAMS \& A. ADAMS, 1856

Famille: Lucinidae FLEMING, 1828

Genre: Lucina BRUGUIERE, 1797

Espèce: Lucina fallax (FORBES, 1846) Fig. 5.6.

1846 Lucina fallax sp. nov. FORBES, p. 143, pl. 17 , fig. 8 .

1871 Lucina fallax FORBES - STOLICZKA, p. 256, pl. 13, figs. $13,15-17$; pl. 14, figs. 3-5, 7-8. 1953 Lucina fallax FORBES - BARONI et al., p. 95 , pl. 6, fig. 1 .

1972 Lucina malwica sp. nov. CHIPLONKLAR \& BADVE, p. 111, pl. 3, fig. 27.

2006 Lucina fallax FORBES - EL QOT, p. 70, pl. 14, figs. 7-8, 9a-b, 10a-b.

2011 Lucina fallax FORBES - AYOUB-HANNAA, p. 120 , pl. 11, figs. 7-8; text-fig. 3.22.
Description: Coquille de forme sub-circulaire, légèrement inéquilatérale à longueur et hauteur presque identiques. Crochet peu pointu et petit, occupant la partie médiane de la coquille. Ornementation absente due au mauvais état de conservation.

Répartition: Cenomanien: Égypte (Abdelhamid \& Azab, 2003; El Qot, 2006; Ayoub-Hannaa, 2011), Jordanie (Berndt, 2002) et Syrie (Conrad, 1852).

Matériel: 10 spécimens trouvés dans l'unité C.

Mesures:

\begin{tabular}{|l|c|c|c|}
\hline $\mathrm{n}=10$ & $\mathrm{H}$ & $\mathrm{L}$ & $\mathrm{H} / \mathrm{L}$ \\
\hline Étendue & $18.23-30.15$ & $17.27-29.56$ & $1.02-1.08$ \\
\hline Moyenne & $21.36-23.42$ & $21-23.13$ & 1.05 \\
\hline
\end{tabular}

Famille: Mactromyidae COX, 1929

Genre: Clisocolus GABB, 1869

Espèce: Clisocolus corrugatus POPENOE, 1937

Fig. 5.7.

1937 Clisocolus corrugatus sp. nov. POPENOE, p. 390, pl. 47, figs. 9, 10, 12 .

2011 Clisocolus corrugatus POPENOE - AYOUBHANNAA, p. 120, pl. 11, fig. 9.

2014 Clisocolus corrugatus POPENOE - AYOUBHANNAA \& FÜRSICH, p. 128, pl. 11, fig. 9.

Description: Coquille moyenne, de forme subarrondie, inéquilatérale, inéquivalve et peu gonflée. Sa longueur est presque égale à sa hauteur. Des nervures légères et concentriques composent l'ornementation. Elles sont séparées par des interstices larges qui deviennent plus étroits dorsalement. Crochet important et fortement incurvé.

Répartition: Crétacé Supérieur: Californie (Popenoe, 1937) et Égypte (Ayoub-Hannaa, 2011; Ayoub-Hannaa \& Fürsich, 2014).

Matériel: 5 spécimens dont 3 récoltés dans l'unité A, et 2 dans la l'unité $C$.

Mesures:

\begin{tabular}{|l|c|c|c|}
\hline $\mathrm{n}=5$ & $\mathrm{H}$ & $\mathrm{L}$ & $\mathrm{H} / \mathrm{L}$ \\
\hline Étendue & $23.77-29.57$ & $20.66-26.88$ & $1.10-1.15$ \\
\hline Moyenne & $24.67-26.51$ & $23.95-24.05$ & 1.13 \\
\hline
\end{tabular}

Famille: Carditidae FLEMING, 1820 (1828)

Genre: Venericardia LAMARCK, 1801

Espèce: Venericardia forgemoli (COQUAND, 1862)

Fig. 5.8. 
1862 Cardita forgemoli sp. nov. COQUAND, p. 199, pl. 14, figs. 14-15.

1862 Cardita delettrei sp. nov. COQUAND, p. 200, pl. 14, figs. 18-19.

1891 Cardita beuquei COQUAND - PÉRON, p. 264.

1912 Cardita Forgemoli COQUAND -

PERVINQUIÈRE, p. 238, pl. 17, figs. 5-12.

1918 Cardita Forgemoli COQUAND - GRECO, p. 32, pl. 4 (20), figs. 4-7.

1937 Cardita Forgemoli COQUAND -

TREVISAN, p. 94, pl. 7, figs. 10-13.

2004b Venericardia forgemoli COQUAND -

ABDEL-GAWAD et al., pl. 2, fig. 10.

2006 Maghrebella forgemoli COQUAND - EL

QOT, p. 73, pl. 15, figs. 6-8.

2007b Maghrebella forgemoli COQUAND -

MEKAWY, p. 228, pl. 5, fig. 1.

2011 Venericardia ?forgemoli COQUAND -

AYOUB-HANNAA, p. 131, pl. 12, fig. 1.

2014 Venericardia forgemoli COQUAND - AYOUB-

HANNAA \& FÜRSICH, p. 101, pl. 10, fig. 1.

Description: Coquille de taille moyenne, à contour triangulaire, inéquilatérale, gonflée, plus haute que longue. Crochet important, relativement large et incliné. Ornementation représentée par des nervures radiales bien marquées et séparées par des interstices étroits.

Discussion: Les similitudes (en terme de contour, de taille et d'ornementation) entre Cardita beuquei (COQUAND, 1862), Cardita delettrei (COQUAND, 1862 ) et Cardita forgemoli amenèrent Pervinquière (1912) à les considérer comme synonymes de Cardita forgemoli.

Répartition: Cénomanien: Algérie (Coquand, 1862), Tunisie (Pervinquière, 1912), Égypte (Greco, 1918; Ayoub-Hannaa, 2011; Ayoub-Hannaa \& Fürsich, 2014) et Italie (Trevisan, 1937).

Matériel: 1 spécimen trouvé dans l'unité C.

Famille: Cardiidae LAMARCK, 1809

Genre: Granocardium GABB, 1868

Espèce: Granocardium carolinum (D’ORBIGNY, 1843) Fig. 5.9.

1843 Cardium carolinum sp. nov. D'ORBIGNY, p. 29 , pl. 245.

1981 Granocardium cf. carolinum D'ORBIGNY AMARD et al., p. 75, pl. 1, figs. 1-2.
2006 Granocardium (?Criocardium) sp.

PERRILLIAT et al., p. 101, figs. 19-24.

2011 Granocardium (Granocardium) carolinum

D'ORBIGNY - AYOUB-HANNAA, p. 131, pl. 12,

figs. 2-3.

2014 Granocardium (Granocardium) carolinum

D'ORBIGNY - AYOUB-HANNAA \& FÜRSICH, p. 113 , pl. 10, figs. 2-3.

Description: Coquille de taille moyenne, souvent ovale et légèrement plus haute que longue. Elle est fortement gonflée, équivalve et légèrement équilatérale. L'ornementation se compose de fils radiaux fins. Des interstices, étroits, se développent près de la marge ventrale. Crochet important, presque central, sub-triangulaire et incurvé postérieurement.

Répartition: Crétacé Supérieur: France (D’Orbigny, 1843). Cénomanien supérieur-Turonien inferieur: Jordanie (Perrilliat et al., 2006). Cénomanien-Turonien: Égypte (Ayoub-Hanna, 2011; Ayoub-Hanna \& Fürsich, 2014).

Matériel: 6 spécimens dont 1 trouvé dans l'unité A et 5 dans l'unité $C$.

Mesures:

\begin{tabular}{|l|c|c|c|}
\hline $\mathrm{n}=6$ & $\mathrm{H}$ & $\mathrm{L}$ & $\mathrm{H} / \mathrm{L}$ \\
\hline Étendue & $10.11-19.28$ & $8.64-18.98$ & $1-1.17$ \\
\hline Moyenne & $14.41-17.05$ & $12.65-13.83$ & 1.14 \\
\hline
\end{tabular}

Espèce: Granocardium desvauxi (COQUAND, 1862) Fig. 5.10.

1862 Cardium desvauxi sp. nov. COQUAND, p. 206, pl. 11, figs. 3-4.

1912 Cardium (Trachycardium) desvauxi COQUAND - PERIVINQUIËRE, p. 260.

1962 Granocardium hassani sp. nov. ABBASS, p. 122 , pl. 20, figs. 2-3.

2011 Granocardium (Granocardium) desvauxi COQUAND - AYOUB-HANNAA, p. 131, pl. 12, fig. 4.

2014 Granocardium (Granocardium) desvauxi COQUAND - AYOUB-HANNAA \& FÜRSICH, p. 114, pl. 10, fig. 4 .

Description: Coquille triangulaire, renflée et presque inéquilatérale. Elle a un côté buccal excavé et court, et un côté anal très oblique et caréné à l'extérieur. Crochet recourbé. Ornementation absente. 
Répartition: Cénomanien: Algérie (Benyoucef et al., 2012), Tunisie (Pervinquière, 1912), et Égypte (Abbass, 1962; Ayoub-Hannaa, 2011; Ayoub-Hannaa \& Fürsich, 2014).

Matériel: 6 spécimens récoltés dans l'unité $\mathrm{C}$. Mesures:

\begin{tabular}{|l|c|c|c|}
\hline $\mathrm{n}=6$ & $\mathrm{H}$ & $\mathrm{L}$ & $\mathrm{H} / \mathrm{L}$ \\
\hline Étendue & $19.22-31.95$ & $14.78-27.78$ & $1.15-1.30$ \\
\hline Moyenne & $22.05-26.60$ & $17.43-21.45$ & $1.20-1.24$ \\
\hline
\end{tabular}

Espèce: Granocardium (Granocardium) productum (J. DE C. SOWERBY, 1832)

Fig. 5.11.

1832 Cardium productum J. DE C. SOWERBY, pl. 12, figs. 5-7; Text-fig. 3.23.

1832 Cardium productum sp. nov. J. DE C.

SOWERBY, p. 417, pl. 39, fig. 15.

1850 Cardium olisiponensis sp. nov. SHARPE, p. 181, pl. 14, figs. 4a-b.

1917 Cardium productum SOWERBY - FOURTAU, p. 81.

1963 Cardium aff. productum SOWERBY - FAWZI, p. 70.

1971 Trachycardium productum SOWERBY -

COLLIGNON, p. 41, pl. H, fig. 10.

1993 Granocardium productum SOWERBY -

DHONDT \& DIENI, p. 229, pl. 14, figs. 4-9b.

2004b Granocardium hassani ABBASS - ABDEL-

GAWAD et al., pl. 2, fig. 11.

2006 Granocardium productum SOWERBY - EL

QOT, p. 77, pl. 16, figs. 2a-b, 3.

2011 Granocardium (Granocardium) productum

SOWERBY - AYOUB-HANNAA, p. 131, pl. 12,

figs. 5-7; text-fig. 3.23.

2014 Granocardium (Granocardium) productum SOWERBY - AYOUB-HANNAA \& FÜRSICH, p. 114, pl. 10, figs. 5-7.

Description: Moule interne de taille assez grande, plus haute que longue, trés convexe. Crochet relativement large et incurvé.

Répartition: Cénomanien: Portugal (Sharpe, 1850; Soares, 1966), Algérie (Benyoucef et al., 2012; Benyoucef et al., 2016), Égypte (Fawzi, 1963; AyoubHannaa, 2011; Ayoub-Hannaa \& Fürsich, 2014) et Italie (Dhondt \& Dieni, 1993). Maestrichtien: Algérie (Collignon, 1971).

Matériel: 1 spécimen récolté dans l'unité C.
Mesures:

\begin{tabular}{|l|c|c|c|}
\hline $\mathrm{n}=1$ & $\mathrm{H}$ & $\mathrm{L}$ & $\mathrm{H} / \mathrm{L}$ \\
\hline Spécimen & 38.46 & 27.47 & 1.40 \\
\hline
\end{tabular}

Famille: Arcticidae NEWTON, 1891

Genre: Tenea CONRAD, 1870

Espèce: Tenea delettrei (COQUAND, 1862) Fig. 6.1.

1862 Venus delettrei sp. nov. COQUAND, p.194, pl. 8, figs. 3-4

1862 Venus forgemoli sp. nov. COQUAND, p. 194, pl. 8, figs. 7-8.

1912 Dosinia delettrei COQUAND -

PERVINQUIÈRE, p. 270, pl. 20, figs. 4-8.

1918 Dosinia delettrei COQUAND - GRECO,

p. 49 (231), pl. 5 (21), figs. 6-10.

1934 Dosinia delettrei COQUAND -

BLANCKENHORN, p. 250.

1937 Dosinia delettrei COQUAND - TREVISAN,

p. 113, pl. 7, figs. 29-31.

1937 Dosinia forgemoli COQUAND - TREVISAN, p. 114, pl. 7, figs. 32-33.

2004b Dosinia delettrei COQUAND - ABDEL-

GAWAD et al., pl. 3, fig. 7.

2006 Tenea delettrei COQUAND - EL QOT, p. 84,

pl. 17, figs. 4-9; text-fig. 11b.

2007b Tenea delettrei COQUAND - MEKAWY, p. 233, pl. 5, fig. 11.

2011 Tenea delettrei COQUAND - AYOUB-

HANNAA, p. 141, pl. 13, figs. 5-7; text-fig. 3.25.

2014 Tenea delettrei COQUAND - AYOUB-

HANNAA \& FÜRSICH, p. 119, pl. 11, figs. 5-7.

Description: Coquille de taille moyenne, de forme ovale et renflée. Elle présente une petite différence entre sa largeur et sa hauteur. Crochet long et étroit par rapport à sa taille. Ornementation mal préservée.

Répartition: Cénomanien: Tunisie (Pervinquière, 1912), Égypte (Greco, 1918; Ayoub-Hannaa, 2011; Ayoub-Hannaa et al., 2014), Italie (Trevisan, 1937) et Syrie (Blackenhorn, 1934). Cénomanien: Algérie (Coquand, 1862).

Matériel: 5 spécimens dont 3 récoltés dans l'unité B et 2 dans l'unité $C$.

\section{Mesures:}

\begin{tabular}{|l|c|c|c|}
\hline $\mathrm{n}=5$ & $\mathrm{H}$ & $\mathrm{L}$ & $\mathrm{H} / \mathrm{L}$ \\
\hline Étendue & $23.77-29.57$ & $15.65-31.80$ & $0.93-1.32$ \\
\hline Moyenne & $24.67-26.51$ & $21.45-24.20$ & $1.10-1.15$ \\
\hline
\end{tabular}




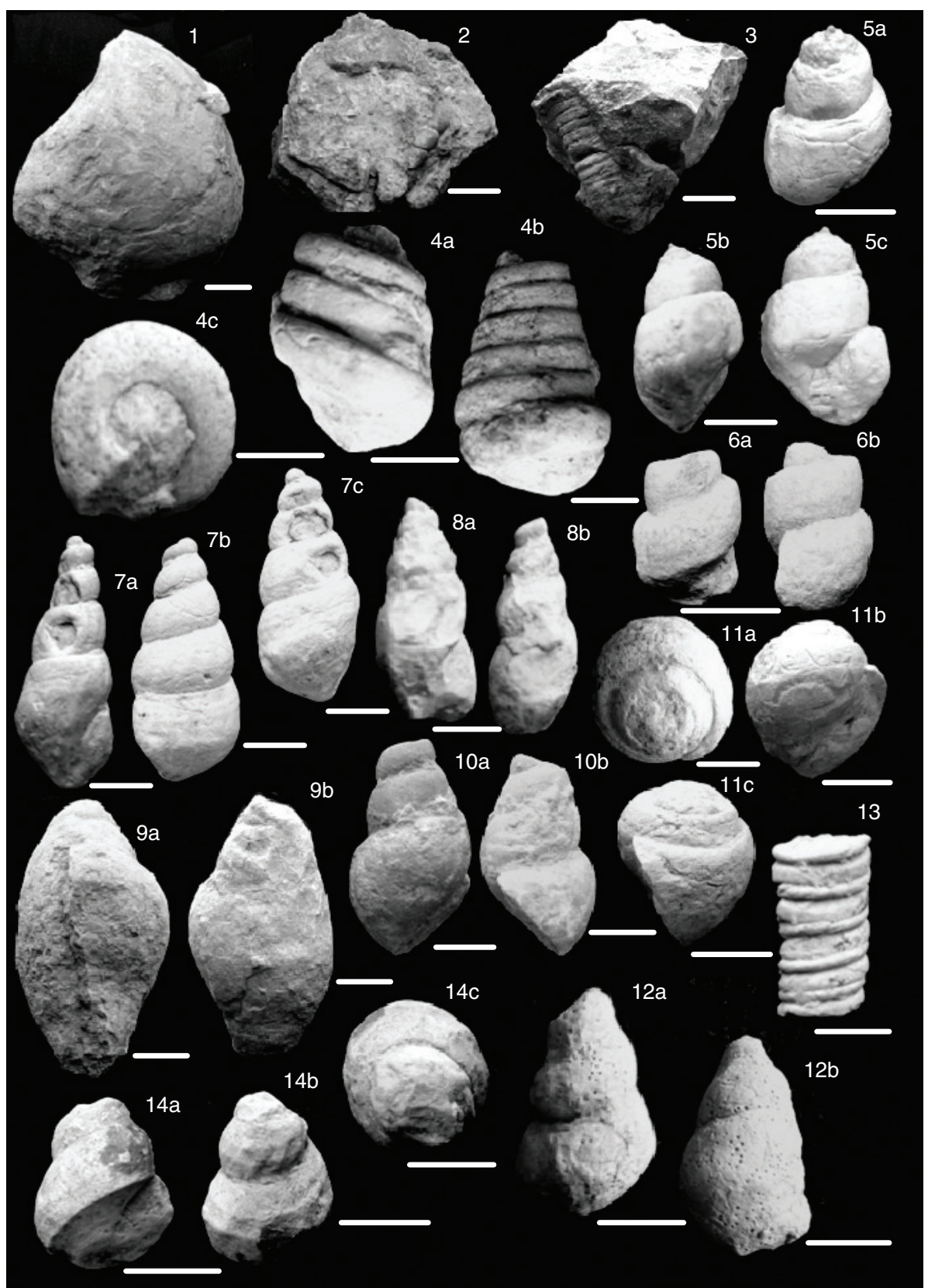

Fig. 6.-1. Tenea delletrei (COQUAND, 1862) de l'unité B. 2. Turritella cf. difficilis (D'ORBIGNY, 1842) de l'unité A. 3. Campanile (Campanile) ganesha (NOETLING, 1897) dans l'unité A. 4. Cimolithium tenouklense (COQUAND, 1862) de l'unité B: 4a- vue dorsale, 4b- vue aperturale, 4c- vue basale. 5. Ampullina? abeihensis (HAMLIN, 1884) dans l'unité C: 5a- vue dorsale, 5b,c- vue aperturale. 6. Aporrhais dutrugei (COQUAND, 1862) de l'unité A: 6a- vue aperturale, 6b-vue dorsale. 7. Aporrhais sp. de l'unité C: 7a, c- vue aperturale, 7b- vue dorsale. 8. Columbellina (Columbellina) fusiformis (DOUVILLÉ, 1916) de l'unité C: 8a- vue aperturale, 8b- vue dorsale. 9. Pterodonta deffisi (THOMAS \& PÉRON, 1889) de l'unité B: 9a- vue aperturale, 9b- vue dorsale. 10. Pterodonta elongata (D'ORBIGNY, 1843) de l'unité A: 10a- vue aperturale, 10b- autre vue aperturale, 11. Tylostoma (Tylostoma) globosum (SHARPE, 1849) de l'unité A. 11a- vue sommitale, 11b- vue aperturale, 11c- vue dorsale. 12. Tylostoma pallaryi (PÉRON \& FOURTAU, 1904) de l'unité C: $12 \mathrm{a}-$ vue aperturale, 12b- vue dorsale. 13. Nerinea texana (ROEMER, 1849) de l'unité B. 14. Nerinea. sp. de l'unité A: 14a- vue aperturale, 14b- vue dorsale. 14c- vue sommitale. (chaque barre correspond à $1 \mathrm{~cm}$ ). 


\section{Gastéropodes}

La classification systématique utilisée est celle de Bouchet \& Rocroi (2005). H, D, HL, HA et WA désignent respectivement la hauteur, le diamètre du spécimen, la hauteur du dernier tour, la hauteur de l'ouverture et sa largeur, tous exprimés en $(\mathrm{mm})$. Le nombre de tours et l'angle pleural sont respectivement abrégés par $\mathrm{Nw}$ et $\mathrm{PA}^{\circ}$.

Famille: Turritellidae LOVÉN, 1847

Genre: Turritella LAMARCK, 1799

Espèce: Turritella cf. difficilis (D'ORBIGNY, 1842) Fig. 6.2.

cf. 1927 Turritella cf. difficilis D'ORBIGNY BLANCKENHORN, p. 141, pl. 3, fig. 37. cf. 1971 Turritella $\mathrm{cf}$. difficilis D'ORBIGNY COLLIGNON, p. 6, pl. B, fig. 1 .

2011 Turritella cf. difficilis D'ORBIGNY AYOUB-HANNAA, p. 165, pl. 15, figs. 5-6.

Description: Moule de petite taille, haute et spiralée, composée de trois tours de recouvrement. Spirales légèrement convexes, séparées par des sutures modérément profondes et obliques. Ornementation non préservée.

Discussion: Selon Blanckenhorn (1927), Turritella cf. difficilis se distingue par son contour général, la convexité des spirales et l'angle de suture.

Répartition: Cénomanien: Égypte (Ayoub-Hannaa, 2011) et Syrie (Blanckenhorn, 1927). Maestrichtien: Algérie (Collignon, 1971).

Matériel: 1 spécimen récolté dans l'unité

Famille: Campanilidae DOUVILLÉ, 1904 Genre: Campanile BAYLE in P. FISCHER, 1884

Espèce: Campanile (Campanile) ganesha (NOETLING, 1897) Fig. 6.3.

1897 Nerinea ganesha sp. nov. NOETLING, p. 58, pl. 15, figs. 1-2; pl. 16, figs. 1-2.

1916 Nerinea ganesha NOETLING-GRECO, p. 121 (63), pl. 15 (7), figs. 10-11.

1971 Nerinea (Ptygmatis) ganesha NOETLING COLLIGNON, p. 17 (159), pl. C, fig. 5.

1974 Campanile (Campanile) ganesha NOETLING ALBANESI \& BUSSON, p. 299.

2006 Campanile (Campanile) ganesha NOETLING EL QOT, p. 98, pl. 19, figs. 12, 14.
2011 Campanile (Campanile) ganesha NOETLING AYOUB-HANNAA, pl. 15, figs. 7-8; text-fig. 3.29.

Description: Moule de taille moyenne, hélicospirale à surface convexe. La spire est constituée de plusieurs tours; le recouvrement est marqué par des sutures peu profondes et se comblant vers l'apex. Ornementation non conservée.

Discussion: Campanile bussoni (ALBANESI \& BUSSON, 1974) récolté en Algérie et Cerithium inauguratum (STOLICZKA, 1902) du désert égyptien, ressemblent beaucoup à notre matériel mais en diffèrent par le nombre de tours (moins nombreux), la taille (moins grande) et les sutures (moins larges et moins profondes).

Répartition: Cénomanien-Maestrichtien: Égypte (Greco, 1916; El Qot, 2006; Ayoub-Hanna, 2011). Maestrichtien: Algérie (Collignon, 1971; Albanesi \& Busson, 1974).

Matériel: 3 spécimens dont 2 trouvés dans l'unité A et 1 dans l'unité $C$.

Ordre et Famille: incertain

Genre: Cimolithium COSSMANN, 1906

Espèce: Cimolithium tenouklense (COQUAND, 1862) Fig. 6.4.

1862 Turritella tenouklensis sp. nov.COQUAND, p. 176, pl. 4, fig. 6 .

1889 Cerithium tenouklense COQUAND - PÉRON, pl. 20, fig. 2.

1912 Cerithium tenouklense COQUAND -

PERVINQUIÈRE, p. 16, pl. 1, figs. 20-22.

1916 Cerithium (Cimolithium) Tenouklense COQUAND

- GRECO, p. 154 (96), pl. 19 (11), figs. 3-5.

1927 Cerithium tenouklense COQUAND BLANCKENHORN, p. 162, pl. 8 (4), figs. 77-80. 2002 Cimolithium tenouklense COQUAND ZAKHERA, p. 310, fig. 5.

2004b Cimolithium tenouklense COQUAND ABDEL-GAWAD et al., pl. 5, fig. 2.

2006 Cimolithium tenouklense COQUAND - EL QOT, p. 97, pl. 19, fig. 8. 2007a Cimolithium tenouklense COQUAND - MEKAWY, p. 157, pl. 1, fig. 5. 2011 Cimolithium tenouklense COQUAND AYOUB-HANNAA, p. 167, pl. 16, figs. 1-3.

Description: Moule comprenant 7 tours arrondis vers le haut et sub-carénés à leur base, avec 
une suture profonde qui les sépare. Canal antérieur médian, court bien distinct.

Discussion: Selon Coquand (1879), cette espèce peut être confondue avec Cerihium gaudae. Elle se distingue par sa forme beaucoup plus allongée, plus grêle, et par un plus grand nombre de tours.

Répartition: Cénomanien: Algérie (Coquand, 1862; Busson et al., 1999; Benyoucef et al., 2017), Tunisie (Pervinquière, 1912), Égypte (Greco, 1916; Ayoub-Hannaa, 2011) et Syrie (Blackenhorn, 1927).

Matériel: 12 spécimens dont 9 récoltés dans l'unité A, 1 dans l'unité B, et 2 dans l'unité C.
Famille: Aporrhaidae GRAY, 1850

Genre: Aporrhais DA COSTA, 1778

Espèce: Aporrhais dutrugei (COQUAND, 1862)

Fig. 6.6.

1862 Rostellaria dutrugei sp. nov. COQUAND, p. 185 , pl. 5 , fig. 4 .

1889 Pterodonta (?) dutrugei COQUAND - PERON, p. 83, pl. 20, figs. 15-16.

1912 Aporrhais (?) dutrugei COQUAND -

PERVINQUIÈRE, p. 24, pl. 2, figs. 1-7.

\section{Mesures:}

\begin{tabular}{|l|c|c|c|c|c|c|c|c|c|c|}
\hline $\mathrm{n}=12$ & $\mathrm{H}$ & $\mathrm{D}$ & $\mathrm{HL}$ & $\mathrm{HA}$ & $\mathrm{WA}$ & $\mathrm{PA}^{\circ}$ & $\mathrm{Nw}$ & $\mathrm{D} / \mathrm{H}$ & $\mathrm{HL} / \mathrm{H}$ & $\mathrm{WA} / \mathrm{HA}$ \\
\hline Etendue & $17-39$ & $15-24$ & $6-11.50$ & $4.50-10.50$ & $5-15$ & $32^{\circ}-42^{\circ}$ & $5-7$ & $0.50-0.82$ & $0.3-0.4$ & $1.10-1.40$ \\
\hline Moyenne & 28 & 18.50 & 8.75 & 7.63 & 8.33 & $37^{\circ}$ & 6 & 0.70 & 0.35 & 1.26 \\
\hline
\end{tabular}

Famille: Ampullinidae COSSMANN, 1919

Genre: Ampullina BOWDICH, 1822

Espèce: Ampullina? abeihensis (HAMLIN, 1884)

Fig. 6.5.

1884 Amauropsis abeihensis sp. nov. HAMLIN, p. 17 , pl. 1, fig. 2 .

1890 Amauropsis abeihensis HAMLIN -

BLANCKENHORN, p. 103.

1927 Natica (Euspira) abeihensis HAMLIN -

BLANCKENHORN, p. 131, pl. 1, figs. 21-22.

1939 Tylostoma (Odostomopis) abeihensis

HAMLIN - DELPEY, p. 80, fig. 55.

2011 Ampullina? abeihensis HAMLIN - AYOUB-

HANNAA, p. 171, pl. 16, figs. 4-5.

Description: Coquille relativement grande, à enroulement bas, composé de trois spires de recouvrement soulignées de sutures assez profondes. Le tour terminal représente deux tiers de la hauteur totale. Spires décroissant rapidement vers l'apex, avec des surfaces droites à légèrement convexes. Lèvre externe plus convexe que la lèvre interne. Apex aigu à base plate. Ornementation mal conservée.

Répartition: Aptien: Syrie et Égypte(Blanckenhorn, 1927; Ayoub-Hannaa, 2011).

Matériel: 1 spécimen trouvé dans l'unité C.
1916 Aporrhais (?) dutrugei COQUAND - GRECO, p. 155 (97).

1927 Pterodonticeras dutrugei COQUAND -

BLANCKENHORN, p. 168, pl. 9 (5), fig. 93.

1937 Aporrhais (?) dutrugei COQUAND -

TREVISAN, p. 35, pl. 2, fig. 8 .

2004b Aporrhais dutrugei COQUAND - ABDEL-

GAWAD et al., pl. 5, fig. 6.

2006 Aporrhais dutrugei COQUAND - EL QOT,

p. 100, pl. 20, figs. 7-8.

2007a Aporrhais dutrugei COQUAND -

MEKAWY, p. 160, pl. 1, fig. 15.

2007a Aporrhais dutrugei COQUAND -

MEKAWY, p. 160, pl. 1, fig. 15.

2011 Aporrhais dutrugei COQUAND - AYOUB-

HANNAA, p. 177, pl. 17, figs. 3-4.

Description: Gastéropode de petite taille, à spire haute composée de deux tours convexes et aplatis, séparés par des sutures moyennement profondes. Ouverture modérée mais étroite. Ornementation totalement absente.

Discussion: Malgré l'attribution de notre spécimen à Aporrhais dutrugei (COQUAND, 1862), il comporte, malgré tout, certaines différences: une forme plus petite, des sutures plus profondes et une forme plus aplatie des tours.

Mesures:

\begin{tabular}{|l|c|c|c|c|c|c|c|c|c|c|}
\hline $\mathrm{n}=1$ & $\mathrm{H}$ & $\mathrm{D}$ & $\mathrm{HL}$ & $\mathrm{HA}$ & $\mathrm{WA}$ & $\mathrm{PA}^{\circ}$ & $\mathrm{Nw}$ & $\mathrm{D} / \mathrm{H}$ & $\mathrm{HL} / \mathrm{H}$ & WA/HA \\
\hline Spécimen & 21 & 18.5 & 17 & 12.50 & 9 & $79^{\circ}$ & 3 & 0.88 & 0.80 & 0.72 \\
\hline
\end{tabular}


Répartition: Cénomanien: Algérie (Coquand, 1862), Tunisie (Pervinquière, 1912), Égypte (Greco, 1916; Ayoub-Hannaa, 2011), Italie (Trevisan, 1937) et Syrie (Blanckenhorn, 1927).

Matériel: 1 spécimen trouvé dans l'unité A.

Mesures:

\begin{tabular}{|l|c|c|c|c|c|c|c|c|c|c|}
\hline $\mathrm{n}=1$ & $\mathrm{H}$ & $\mathrm{D}$ & $\mathrm{HL}$ & $\mathrm{HA}$ & $\mathrm{WA}$ & $\mathrm{PA}^{\circ}$ & $\mathrm{Nw}$ & $\mathrm{D} / \mathrm{H}$ & $\mathrm{HL} / \mathrm{H}$ & $\mathrm{WA} / \mathrm{HA}$ \\
\hline Spécimen & 15 & 10.50 & 9 & 7 & 4 & $43^{\circ}$ & 3 & 0.70 & 0.60 & 0.44 \\
\hline
\end{tabular}

Espèce: Aporrhais sp.

Fig. 6.7.

Description: Gastéropode de taille moyenne, haut et spiralé. Spire composée de cinq tours convexes, séparés par des sutures peu profondes. Ouverture
Discussion: Une silhouette fusiforme ovée à ouverture ovale, une base pointue et des nervures axiales aux fines cordes spiralées sont les caractéristiques principales de Columbellina fusiformis.
Répartition: Cénomanien: Égypte (Douvillé, 1916; Ayoub-Hannaa, 2011) et Jordanie (Berndt, 2002).

Matériel: 1 spécimen trouvé dans l'unité C.

Genre: Pterodonta D’ORBIGNY, 1842

Mesures:
\begin{tabular}{|l|c|c|c|c|c|c|c|c|c|c|}
\hline $\mathrm{n}=1$ & $\mathrm{H}$ & $\mathrm{D}$ & $\mathrm{HL}$ & $\mathrm{HA}$ & $\mathrm{WA}$ & $\mathrm{PA}^{\circ}$ & $\mathrm{Nw}$ & $\mathrm{D} / \mathrm{H}$ & $\mathrm{HL} / \mathrm{H}$ & $\mathrm{WA} / \mathrm{HA}$ \\
\hline Spécimen & 25 & 13 & 12 & 9 & 5 & $55^{\circ}$ & 4 & 0.52 & 0.48 & 0.55 \\
\hline
\end{tabular}

longue et étroite, ovale à lèvre externe inachevée.

Ornementation absente.

Matériel: 1 spécimen récolté dans l'unité C.

Mesures:

\begin{tabular}{|l|c|c|c|c|c|c|c|c|c|c|}
\hline $\mathrm{n}=1$ & $\mathrm{H}$ & $\mathrm{D}$ & $\mathrm{HL}$ & $\mathrm{HA}$ & $\mathrm{WA}$ & $\mathrm{PA}^{\circ}$ & $\mathrm{Nw}$ & $\mathrm{D} / \mathrm{H}$ & $\mathrm{HL} / \mathrm{H}$ & $\mathrm{WA} / \mathrm{HA}$ \\
\hline Spécimen & 31 & 14 & 17 & 10 & 4 & $50^{\circ}$ & 5 & 0.45 & 0.55 & 0.40 \\
\hline
\end{tabular}

Famille: Colombellinidae FISCHER, 1884

Genre: Columbellina D'ORBIGNY, 1842

Espèce: Columbellina (Columbellina) fusiformis (DOUVILLÉ, 1916) Fig. 6.8.

1916 Columbellina fusiformis sp. nov. DOUVILLÉ, p. 132, pl. 18, figs. 1-3.

2002 Columbellina fusiformis DOUVILLÉ -

BERNDT, p. 144, pl. 9, fig. 8 .

2004b Columbellina (Columbellina) fusiformis

DOUVILLÉ - ABDEL-GAWAD et al., pl. 5, fig. 9.

2011 Columbellina (Columbellina) fusiformis

DOUVILLÉ - AYOUB-HANNAA, p. 134, fig. 13.

Description: Coquille conique fusiforme modérément haute. Quatre spires de recouvrement légèrement convexes. Sutures peu profondes. Apex interrompu. Base presque pointue. Ouverture étroite et légèrement ovale. Ornementation éffacée.
Espèce: Pterodonta deffisi (THOMAS \& PÉRON, 1889)

Fig. 6.9. 
presque aigue. Ouverture relativement étroite se prolongeant en une forme ovale. Ornementation absente.

Discussion: Pterondata subinflata (COQUAND, 1862) et Pterondata inflata (D'OBRIGNY, 1842) différent de Pterondata deffisi par une forme plus gonflée, des lèvres internes et externes fortement convexes avec une base presque arrondie.

Répartition: Cénomanien: Égypte (El Qot, 2006; Ayoub-Hannaa, 2011), Syrie (Blanckenhorn, 1934) et Jordanie (Berndt, 2002).

Matériel: 1 spécimen trouvé dans l'unité B.

Mesures:

\begin{tabular}{|l|c|c|c|c|c|c|c|c|c|c|}
\hline $\mathrm{n}=1$ & $\mathrm{H}$ & $\mathrm{D}$ & $\mathrm{HL}$ & $\mathrm{HA}$ & $\mathrm{WA}$ & $\mathrm{PA}^{\circ}$ & $\mathrm{Nw}$ & $\mathrm{D} / \mathrm{H}$ & $\mathrm{HL} / \mathrm{H}$ & $\mathrm{WA} / \mathrm{HA}$ \\
\hline Spécimen & 62 & 35 & 51 & 42 & 18 & $50^{\circ}$ & 4 & 0.56 & 0.82 & 0.43 \\
\hline
\end{tabular}

Espèce: Pterodonta elongata (D’ORBIGNY, 1843)

Fig. 6.10.

1843 Pterodonta elongata n. sp. D’ORBIGNY, p. 316, pl. 218, fig. 2 .

1843 Pterodonta intermedia D'ORBIGNY, p. 319 , pl. 220, fig. 1.

?1843 Pterodonta ovata D’ORBIGNY, p. 317, pl. 218 , fig. 3.

?1974 Pterodonta ovata D'ORBIGNY -

ALBANESI \& BUSSON, pl. 24, fig. 1.

1985 Pterodonta elongata D'ORBIGNY -

KOLLMANN, p. 97, fig. 5f-g.

2015 Pterodonta elongata D'ORBIGNY -

AYOUB-HANNAA et al., p. 48, fig. 10 (G-I).

Description: Coquille allongée, composée de trois tours assez convexes peu aplatis. Le dernier tour représente presque les deux tiers de la taille de la coquille. Ouverture assez longue et étroite. Ornementation absente.

Discussion: Pterodonta elongata ressemble beaucoup à Pterodonta ovata, mais cette dernière s'en différencie clairement par une forme plus raccourcie et une dent prolongée sur le labre.

Répartition: Cénomanien: Algérie (Albanesi \& Busson, 1974) et Serbie centrale (Ayoub-Hannaa et al., 2015). Cénomanien-Santonien supérieur: France (D’Orbigny, 1843; Kollmann, 2005),

Matériel: 1 spécimen trouvé dans l'unité A.

Mesures:

\begin{tabular}{|l|c|c|c|c|c|c|c|c|c|c|}
\hline $\mathrm{n}=1$ & $\mathrm{H}$ & $\mathrm{D}$ & $\mathrm{HL}$ & $\mathrm{HA}$ & $\mathrm{WA}$ & $\mathrm{PA}^{\circ}$ & $\mathrm{Nw}$ & $\mathrm{D} / \mathrm{H}$ & $\mathrm{HL} / \mathrm{H}$ & $\mathrm{WA} / \mathrm{HA}$ \\
\hline Spécimen & 29 & 17 & 21 & 13 & 10 & $50^{\circ}$ & 4 & 0.59 & 0.72 & 0.77 \\
\hline
\end{tabular}

Famille: Tylostomatidae STOLICZKA, 1868

Espèce: Tylostoma (Tylostoma) globosum

(SHARPE, 1849) Fig. 6.11.

1849 Tylostoma globosum sp. nov. SHARPE, p. 379 , pl. 9, figs. 5-6.

1912 Tylostoma globosum SHARPE -

PERVINQUIÈRE, p. 53, pl. 4, figs. 9-10. p. 143 (85), pl. 17 (9), figs. 11-12.
1916 Tylostoma globosum SHARPE - GRECO,
1974 Tylostoma (Tylostoma) globosum

SHARPE - ALBANESI \& BUSSON, p. 309, pl. 25 , fig. 1.

1981 Tylostoma globosum SHARPE - AMARD

et al., p. 70, pl. 5, fig. 3.

1985 Tylostoma aff. globosa SHARPE -

KOLLMANN, p. 102, fig. 5k.

2001 Tylostoma globosum SHARPE - KORA et al., pl. 3 , fig. 4.

2002 Tylostoma globosum SHARPE - BERNDT, p. 143 , pl. 9 , fig. 5 .

2011 Tylostoma (Tylostoma) globosum SHARPE -

AYOUB-HANNAA, p. 137, fig. 14.

Description: Grand gastéropode, globuleux à spire basse. Spire comportant 3 tours arrondis et presque lisses. Les spires sont larges, comprimées et séparées par des sutures légèrement déprimées. Base largement arrondie à ouverture étroite semi-lunaire et lèvre externe largement plus convexe que l'interne. Ornementation absente.

Discussion: Tylostoma cossoni (THOMAS \& PERON, 1889) diffère de notre matériel par une spire relativement plus haute.

Répartition: Cénomanien: Jordanie(Berndt, 2002). limite Cénomanien-Turonien: Algérie (Albanesi \& Busson, 1974; Amard, et al., 1981; Busson et al., 1999; Benyoucef et al., 2017). Turonien: Portugal (Sharpe, 
1849), Tunisie (Pervinquière, 1912) et Égypte (Kora et al., 2001; Ayoub-Hannaa, 2011). Santonien: France (Kollmann, 1985).

Matériel: 1 spécimen trouvé dans l'unité A. Mesures:
Famille: Nerineidae ZITTEL, 1873

Genre: Nerinea DEFRANCE, 1825

Espèce: Nerinea texana (ROEMER, 1849)

\begin{tabular}{|l|c|c|c|c|c|c|c|c|c|c|}
\hline $\mathrm{n}=1$ & $\mathrm{H}$ & $\mathrm{D}$ & $\mathrm{HL}$ & $\mathrm{HA}$ & $\mathrm{WA}$ & $\mathrm{PA}^{\circ}$ & $\mathrm{Nw}$ & $\mathrm{D} / \mathrm{H}$ & $\mathrm{HL} / \mathrm{H}$ & $\mathrm{WA} / \mathrm{HA}$ \\
\hline Spécimen & 24 & 20 & 19 & 17 & 10 & $100^{\circ}$ & 4 & 0.83 & 0.79 & 0.59 \\
\hline
\end{tabular}

Espèce: Tylostoma pallaryi (PÉRON \&

FOURTAU, 1904) Fig. 6.12.

1904 Pseudomelania pallaryi sp. nov.

PÉRON \& FOURTAU in FOURTAU, p. 270 , pl. 1, fig. 22.

1916 Tylostoma pallaryi PÉRON \& FOURTAU - GRECO, p. 151 (93), pl. 18 (10),

figs. 2-4.

1963 Tylostoma pallaryi PÉRON \& FOURTAU FAWZI, p. 91, pl. 7, figs. 1-3.

2004 Tylostoma pallaryi PÉRON \&

FOURTAU - ABDEL-GAWAD et al., pl. 5,

fig. 13.

2011 Tylostoma pallaryi PÉRON \& FOURTAU AYOUB-HANNAA, p. 138.

Description: Spécimen de taille modérément large, haut et spiralé. La spire est formée de trois tours assez convexes et séparés par des sutures relativement déprimées. Dernier tour large, à flancs convexes, de orme semi-conique occupant plus de la moitié de la hauteur totale. Ouverture relativement étroite. Ornementation totalement absente.

Discussion: Tylostoma syriacum (CONRAD, 1852) du Cénomanien syrien ressemble à notre matériel mais en diffère par sa forme générale et ses sutures plus profondes.

Répartition: Cénomanien: Algérie (Péron \& Fourtau, 1904; Busson et al., 1999; Benyoucef et al., 2017), l'Egypte (Greco, 1916; Ayoub-Hannaa, 2011). Maestrichtien: Arabie Saoudite (Gameil \& El-Sorogy, 2015).

Matériel: 1 spécimen trouvé dans l'unité C. Mesures:
Fig. 6.13.

1849 Nerinea sp. ROEMER, p. 412.

1852 Nerinea texana ROEMER, p. 41, pl. 4, fig. 7. 1928 Nerinea texana ROEMER - ADKINS, p. 187.

Description: Coquille allongée, formée d'alternance de tours étroits avec d'autres plus larges. Le dernier tour est un peu évidé en dessus.

Discussion: Nerinea texana (Roemer, 1849) ressemble beaucoup à Nerinea visurgis, mais s'en distingue par une coquille plus étroite et moins carènée.

Matériel: 1 spécimen récolté dans l'unité B.

\section{Espèce: Nerinea sp.}

Fig. 6.14.

Description: Coquille de très petite taille, incomplète, fusiforme, se composant de deux spires légèrement convexes. Ornementation constituée de cordes spiralées et de nervures axiales.

Matériel: 2 spécimens trouvés dans l'unité A.

Ordre: Cephalaspidea FISCHER, 1883

Famille: Ringiculidae PHILIPPI, 1853

Genre: Avellana D'ORBIGNY, 1843

Espèce: Avellana cassis (D'ORBIGNY, 1842)

Fig. 7.1.

1842 Cassis avellana D’ORBIGNY, pl. 169, figs. $10-15$.

Description: Petite coquille formée de deux tours peu convexes dont le dernier occupe la majorité du volume. Base presque aiguë, à ouverture longue et étroite. Ornementation absente.

\begin{tabular}{|l|c|c|c|c|c|c|c|c|c|c|}
\hline $\mathrm{n}=1$ & $\mathrm{H}$ & $\mathrm{D}$ & $\mathrm{HL}$ & $\mathrm{HA}$ & $\mathrm{WA}$ & $\mathrm{PA}^{\circ}$ & $\mathrm{Nw}$ & $\mathrm{D} / \mathrm{H}$ & $\mathrm{HL} / \mathrm{H}$ & $\mathrm{WA} / \mathrm{HA}$ \\
\hline Spécimen & 22 & 14 & 15 & 12 & 9 & $60^{\circ}$ & 3 & 0.64 & 0.68 & 0.75 \\
\hline
\end{tabular}




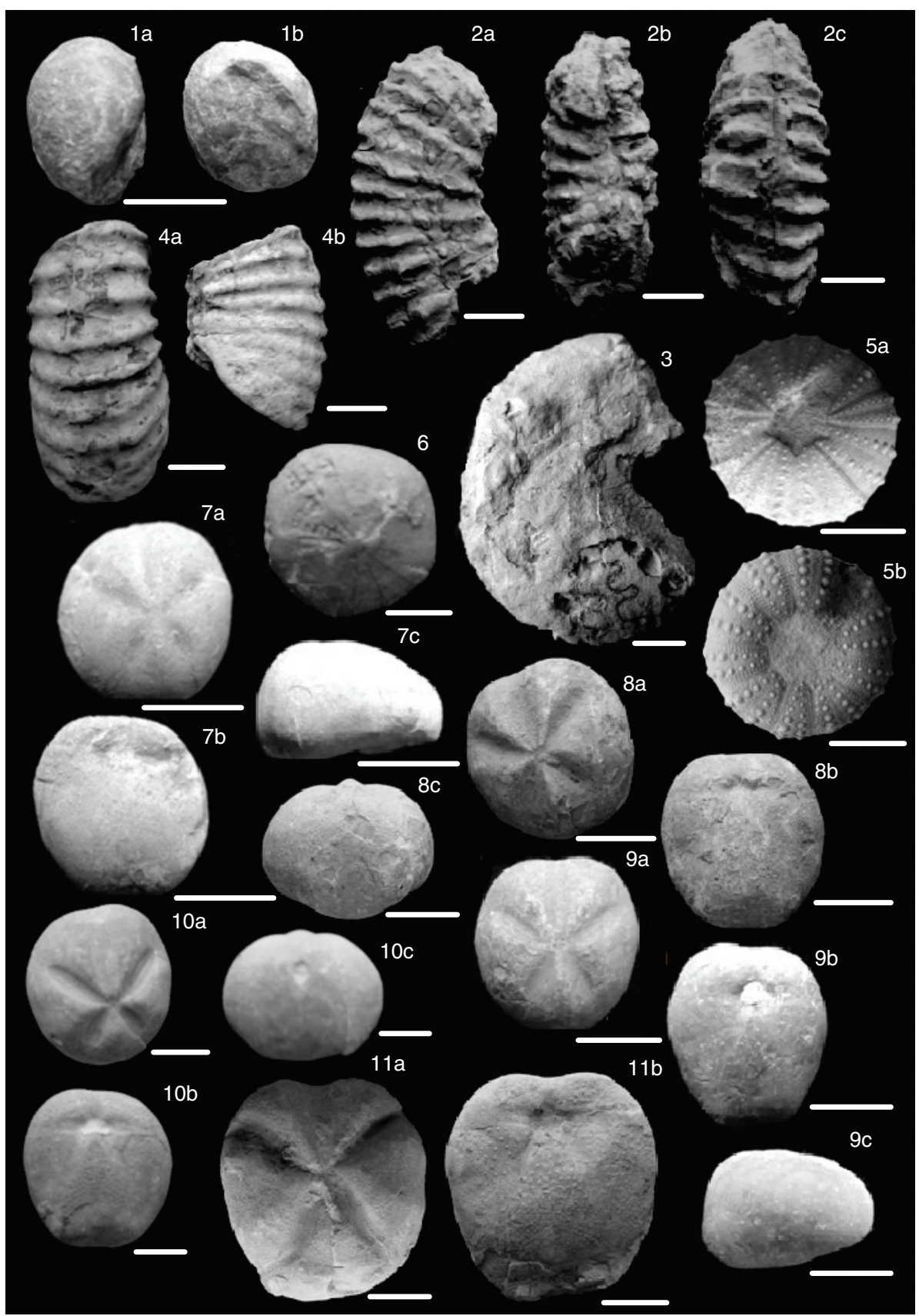

Fig. 7.-1. Avellana cassis (D'ORBIGNY, 1842) de l'unité A: 1a-vue aperturale, 1b-vue dorsale. 2. Sharpeiceras laticlavium (SHARPE, 1855) de l'unité A: 2a- vue de coté, 2b- vue dorsale, 2c- vue ventrale. 3. Neolobites vibrayeanus (D'ORBIGNY, 1841) de l'unité C. 4. Acanthoceras rhotomagense (BRONGNIART, 1822) de l'unité B: 4a- vue ventrale, 4b- vue de coté. 5. Heterodiadema libycum (AGASSIZ \& DESOR, 1846) de l'unité A: 5a- vue apicale, 5b-vue adorale. 6. Coenholectypus excisus (DESOR, 1847) de l'unité C. 7. Hemiaster (Hemiaster) syriacus (CONRAD, 1852) de l'unité A: 7a- vue apicale, 7b- vue adorale, 7c- vue du périprocte. 8. Hemiaster gabrielis (PÉRON \& GAUTHIER, 1878) de l'unité A: 8a- vue apicale, 8b- vue adorale, 8c- vue du périprocte. 9. Hemiaster (Mecaster) heberti (COQUAND, 1862) de l'unité D: 9a- vue apicale, 9b- vue adorale, 9c- vue du coté. 10. Hemiaster (Mecaster) pseudofourneli (PÉRON \& GAUTHIER, 1878) de l'unité A: 10a- vue apicale, 10b- vue adorale, 10c- vue du périprocte. 11. Hemiaster (Mecaster) batnensis (COQUAND, 1862) de l'unité A: 11a- vue apicale, 11b- vue adorale. (chaque barre correspond à $1 \mathrm{~cm}$ ). 
Discussion: Cette espèce se confond aisément avec Avellana incrassata, mais la forme plus renflée marque bien la dernière espèce.

Matériel: 1 spécimen récolté dans l'unité A.

\section{Céphalopodes}

La taxonomie utilisée pour la classification des ammonites collectées suit celle de Wright et al. (1996).

Ordre: Ammonoidea ZITTEL, 1884

Famille: Acanthoceratidae GROSSOUVRE, 1894

Genre: Sharpeiceras HYATT, 1903

Espèce: Sharpeiceras laticlavium (SHARPE, 1855) Fig. 7.2.

1855 Ammonites laticlavius SHARPE, p. 31, pl. 14, fig. 1.

1987 Sharpeiceras laticlavium SHARPE -

WRIGHT \& KENNEDY, p. 127, pl. 41, fig. 4, text-figs. 29, 30, 34a.

1991 Sharpeiceras laticlavium SHARPE -

DELAMETTE \& KENNEDY, p. 454, figs. 9.9, 9.10.

2010 Sharpeiceras laticlavium SHARPE -

AMEDRO \& ROBASZYNSKI, p. 13, pl. 1, fig. 1.

2012 Sharpeiceras laticlavium SHARPE -

AMEDRO et al., p. 11, pl. 3, fig. 2.

2015 Sharpeiceras laticlavium SHARPE -

KENNEDY et al., p. 11, fig. 12R.

2015 Sharpeiceras laticlavium SHARPE -

KENNEDY \& GALE, p. 274, pl. 10, fig. 3.

Description: Spécimen incomplet montrant néanmoins une section presque rectangulaire à costulation dense et à croissance rapide.

Répartition: Cénomanien: Angleterre nord-occidentale et Sud-Est de la France (Amédro et al., 2012), Roumanie, Tunisie, Iran, Madagascar et Afrique du sud (Kennedy et al., 2011, 2015).

Matériel: 1 spécimen récolté dans l'unité A.

Genre: Neolobites FISCHER, 1882

Espèce: Neolobites vibrayeanus (D’ORBIGNY, 1841) Fig. 7.3.

1841 Ammonites vibrayeanus sp. nov. D'ORBIGNY, p. 322, pl. 96, figs. 1, 3 . 1914 Neolobites vibrayeanus D'ORBIGNY - ECK, p. 185.
1914 Neolobites schweinfurthi ECK - ECK, p. 186, pl. 11, fig. 1.

1914 Neolobites brancai ECK - ECK, p. 188, pl. 12, fig. 1.

1981 Neolobites vibrayeanus D'ORBIGNY -

KENNEDY \& JUIGNET, p. 23, figs. 3-4, 6a; text-fig. 5.

1992 Neolobites vibrayeanus D'ORBIGNY MEISTER et al., p. 59, pl. 1, figs. 1-4, 6;

text-fig. 8.

2002 Neolobites vibrayeanus D'ORBIGNY -

BERNDT, pl. 13, figs. 2-5.

2005 Neolobites vibrayeanus vibrayeanus

D'ORBIGNY - MEISTER \& ABDALLAH, p. 123, pl. 1, figs. 3-6, 8; pl. 2, figs. 2-3, 6 .

2005 Neolobites vibrayeanus D'ORBIGNY -

WIESE \& SCHULZE, p. 933, figs. 4A-E; 5A-D;

6AJ; 7A-E; 8A, B, H, 9A, B, D.

2011 Neolobites vibrayeanus (D'ORBIGNY) -

AYOUB-HANNAA, p. 66, text-fig. 9.

Description: Forme mal conservée qui présente néanmoins les sutures typiques de l'espèce, ces dernières se chevauchent et constituent des lobes étroits (trois lobes distincts sur notre spécimen), lancéolés et largement espacés.

Répartition: Cénomanien terminal: France (D’Orbigny, 1841; Kennedy \& Juignet, 1981), Maroc (Meister \& Rhalmi, 2002; Meister et al., 2017), Algérie (Péron, 1897; Amédro et al., 1996; Benyoucef et al., 2012, 2017), Tunisie (Meister \& Abdallah, 2005), Égypte (Wiese \& Schulze, 2005; Ayoub-Hannaa, 2011), Niger (Meister et al., 1992) et Jordanie (Berndt, 2002).

Matériel: 1 spécimen trouvé dans l'unité C.

Genre: Acanthoceras NEUMAYR, 1875

Espèce: Acanthoceras rhotomagense (BROGNIART, 1822) Fig.7.4.

1822 Ammonites rhotomagensis BRONGNIART, p 83, pl. 6, fig. 2.

1826 Ammonites hippocastanum SOWERBY, p. 23, pl. 514 , fig. 2.

1853 Ammonites rhotomagensis BRONGNIART SHARPE, p. 33, pl. 16, figs. 1-4.

1987 Acanthoceras rhotomagense BRONGNIART WRIGHT \& KENNEDY, p. 156, text-figs. 47-54, 
63-69; pl. 42, fig. 8; pl. 44, figs 1-11; pl. 45, figs. 1-5; pl. 46, figs. 1-4.

2011 Acanthoceras rhotomagense BRONGNIART KENNEDY et al., p. 225, figs. 14A, B, 15A-C.

Description: De forme robuste à tours épais, ornée de nombreuses côtes, longues et courtes en alternance.

Répartition: Cénomanien moyen: France et Belgique (Kennedy et al., 2011)

Matériel: 1 spécimen récolté dans l'unité B.

\section{Échinides}

La classification suivie est celle de Kroh \& Smith (2010). H et L représentent respectivement la hauteur et la longueur du spécimen en $(\mathrm{mm})$.

Ordre: Phymosomatoida MORTENSEN, 1904 Famille: Heterodiadematidae SMITH \& WRIGHT, 1993

Genre: Heterodiadema COTTEAU, 1846

Espèce: Heterodiadema libycum (AGASSIZ \& DESOR, 1846) Fig. 7.5.

1846 Hemicidaris libyca sp. nov. AGASSIZ \& DESOR, p. 338.

1859 Pseudodiadema martinianum, COTTEAU, p. 17 , pl. m, fig. 5.

1921 Heterodiadema libycum AGASSIZ \& DESOR - FOURTAU, p. 16.

1925 Heterodiadema libycum AGASSIZ \& DESOR

- BLANCKENHORN, p. 85, pl. 7, fig. 1a-c. 1985 Heterodiadema lybicum AGASSIZ \& DESOR - BANDEL \& GEYS, p. 106, pl. 4, figs. 6-7; pl. 5, figs. 1-2.

1989 Heterodiadema lybicum AGASSIZ \& DESOR - GEYS, p. 129, pl. 1, figs. 5-6. 1995 Heterodiadema lybicum AGASSIZ \& DESOR - NÉRAUDEAU, p. 406, fig. 3e. 2002 Heterodiadema lybicum AGASSIZ \& DESOR - AHMAD \& ABDELHAMID, p. 462, figs. 5/11-12.

2003 Heterodiadema libycum AGASSIZ \& DESOR - BERNDT, p. 78, figs. 3/1-2. ?2006 Heterodiadema buhaysensis SMITH - EL QOT, p. 130, pl. 30, fig. 5a-c.

2007 Heterodiadema libycum AGASSIZ \&

DESOR - ABDEL-GAWAD et al., pl. 6, fig. 6.
2011 Heterodiadema libycum AGASSIZ \& DESOR AYOUB-HANNAA, p. 248, pl. 31, figs. 2-4.

2012 Heterodiadema libycum AGASSIZ \&

DESOR - BENYOUCEF et al., pl. 2, fig. 4a-b.

Description: Espèce de taille moyenne, de forme sub-circulaire à légèrement pentagonale. Plus ou moins renflée en dessus, et presque plate en dessous. Zones porifères droites un peu déprimées. Elles sont composées, sur leur totalité, de pores simples, arrondis, superposés, ne se multipliant pas près du péristome.

Discussion: Dans le synopsis des échinides fossiles, cette espèce est rapportée raisonnablement à Pseudodiadema avec lesquels elle montre plus d'affinité qu'avec Hemicidaris.

Répartition: Cénomanien: France (Coquand, 1862), Nigeria (Néraudeau \& Courville, 1997), Égypte (Geys, 1989; Ayoub-Hannaa, 2011; Ayoub-Hannaa \& Fürsich, 2011), Jordanie centrale (Bandel \& Geys, 1985; Ahmed \& Abdelhamid, 2002; Brendt, 2003), Arabie Saoudite (Néraudeau, 1995), Algérie (Coquand, 1862; Busson et al., 1999; Benyoucef et al., 2012, 2017), Oman et Émirats Arabes Unis (Smith, 1995).

Matériel: 25 spécimens dont 7 récoltés dans l'unité A, 15 dans l'unité C et 3 dans l'unité D.

\section{Mesures:}

\begin{tabular}{|l|c|c|c|}
\hline $\mathrm{n}=25$ & $\mathrm{H}$ & $\mathrm{L}$ & $\mathrm{L} / \mathrm{H}$ \\
\hline Étendue & $6.75-21.87$ & $16.82-38.76$ & $1.35-2.42$ \\
\hline Moyenne & $12.95-14.49$ & $25.30-27.46$ & $1.80-1.94$ \\
\hline
\end{tabular}

Ordre: Holectypoida DUNCAN, 1889

Famille: Holectypidae LAMBERT, 1900

Genre: Coenholectypus POMEL, 1883

Espèce: Coenholectypus excisus (DESOR, 1847)

Fig. 7.6.

1847 Discoidea excisa DESOR - AGASSIZ \& DESOR, p. 90.

1861 Holectypus excisus DESOR - COTTEAU \& TRIGER, p. 368, pl. 62, figs. 1-4.

1873 Holectypus excisus DESOR - LARLET, p. 81. 1921 Holectypus excisus DESOR - FOURTAU, p. 55.

1989 Caenholectypus excisus DESOR - ZAGHBIBTURKI, p. 72.

1989 Caenholectypus excisus DESOR NERAUDEAU \& MOREAU, p. 312. 
2001 Caenholectypus excisus DESOR ABDELHAMID \& EL QOT, p. 21, fig. 5O-P. 2003 Caenholectypus excisus DESOR ABDELHAMID \& AZAB, p. 864, pl. 4, figs. A-B. 2006 Coenholectypus excisus DESOR - EL QOT, p. 142, pl. 32, fig. 10a-c.

2012 Coenholectypus excisus DESOR BENYOUCEF et al., pl. 2, fig. 2a-b.

Description: Espèce de taille moyenne, subcirculaire, aussi large que longue. La face supérieure est convexe sub-déprimée. La face inférieure, renflée et arrondie sur les bords, est profondément concave en son milieu. Péristome sub-circulaire, peu elliptique. Périprocte assez grand et pointu aux deux extrémités. Zones porifères composées de pores obliques, serrés et très petits vers le sommet, plus espacés à la face inférieure.

Discussion: Cohenholectypus excisus se distingue nettement de ses congénères par la forme générale, la nature et la disposition de ses tubercules, la structure de son péristome, mais surtout par la grandeur de son périprocte qui remonte à la face supérieure jusqu'au tiers de l'interambulacre impair.

Répartition: Cénomanien: Algérie (Benyoucef et al., 2012), Tunisie (Zaghbib-Turki, 1989), Égypte (Abdelhamid \& El Qot, 2001; Abdelhamid \& Azab, 2003; El Qot, 2006) et Palestine (Larlet, 1873).

Matériel: 1 spécimen récolté dans l'unité C.

Ordre: Spatangoida CLAUS, 1876

Famille: Hemiasteridae CLARK, 1917

Genre: Hemiaster AGASSIZ, in AGASSIZ \&

DESOR, 1847

Espèce: Hemiaster (Hemiaster) syriacus (CONRAD, 1852) Fig. 7.7.

1852 Holaster syriacus sp. nov. CONRAD in LYNCH, p. 212, pl. 1, fig. 2.

1925 Hemiaster syriacus CONRAD -

BLANCKENHORN, p. 103, pl. 8, figs. 33-35.

1990 Hemiaster syriacus (CONRAD) - SMITH \&

WRIGHT, p. 61, figs. 19a-b, 20.

?1995 Mecaster orbignyanus (DESOR) -

NÉRAUDEAU, p. 419, fig. 6c.

2001 Hemiaster syriacus (CONRAD) -

ABDELHAMID \& EL QOT, p. 28, fig. 7L.

2003 Hemiaster syriacus (CONRAD) -

ABDELHAMID \& AZAB, p. 872, pl. 5, figs. R, P.
2003 Hemiaster (Mecaster) syriacus (CONRAD) BERNDT, p. 84, fig. 4/1a-c.

2006 Hemiaster (Hemiaster) syriacus (CONRAD) EL QOT, p. 150, pl. 34, fig. 7.

2010 Hemiaster (Hemiaster) syriacus (CONRAD) EL QOT, p. 290, pl. 5, fig. 3.

2011 Hemiaster (Hemiaster) syriacus (CONRAD) AYOUB-HANNAA, pl. 33, figs. 5-6.

Description: Espèce de petite taille, de forme subcirculaire. Sa hauteur maximale représente la moitié du diamètre postérieur. Surface apicale, légèrement convexe, avec un ambulacre frontal assez long. Surface adorale assez aplatie. Ambulacres en forme de rose. Péristome petit et arqué. Périprocte petit et ovale, se trouvant dans le dernier quart de la hauteur maximale à partir de la base du spécimen.

Répartition: Cénomanien: Égypte (Abdelhamid \& Azab, 2003; El Qot, 2006, 2010; Ayoub-Hannaa, 2011), Jordanie (Berndt, 2003), la Syrie (Conrad, 1852), Arabie saoudite (Néraudeau, 1995), et Oman (Smith \& Wright, 1990).

Matériel: 3 spécimen trouvés dans l'unité A.

Espèce: Hemiaster gabrielis (PÉRON \& GAUTHIER, 1878) Fig. 7.8.

1878 Hemiaster gabrielis PÉRON \& GAUTHIER, p. 116, pl. 4, figs. 9-12.

1914 Hemiaster gabrielis PÉRON \& GAUTHIER var. aegyptiaca var. nov. - FOURTAU, p. 74, pl. 6, fig. 7.

1921 Hemiaster gabrielis PÉRON \& GAUTHIER var. aegyptiaca var. nov. - FOURTAU, p. 87.

1932 Hemiaster gabrielis PÉRON \& GAUTHIER LAMBERT, p. 125.

2004a Hemiaster (Hemiaster) gabrielis PÉRON \& GAUTHIER - ABDEL-GAWAD et al., pl. 10, figs. 10-11.

2011 Hemiaster gabrielis PÉRON \& GAUTHIER AYOUB-HANNAA, p. 269, pl. 34, figs 1-2.

Description: Espèce assez déprimée, aussi large que longue, la plus grande hauteur est en arrière du sommet et la plus grande largeur est en avant. Dessus un peu convexe et incliné vers la partie antérieure en pente douce. Dessous légèrement convexe. Les pores, inégaux avec un granule intermédiaire, sont de plus en plus allongés à mesure qu'ils s'éloignent du sommet. 
Discussion: Selon Blackenhorn (1925), Smith (1990) et autres, Hemiaster gabrielis recueilli en Algérie et Hemiaster gabrielis var. egyptiaca du Cénomanien du Sinaï en Égypte (Fourtau, 1914) sont synonymes de Hemiaster syriacus.

Répartition: Cénomanien: Maroc (Lambert, 1932), Algérie (Slami, 2014) et Égypte (Fourtau, 1914, 1921; Ayoub-Hannaa, 2011).

Matériel: 45 spécimens dont 20 individus trouvés dans l'unité A, et 25 spécimens dans l'unité C.

\section{Mesures:}

\begin{tabular}{|l|c|c|c|}
\hline $\mathrm{n}=45$ & $\mathrm{H}$ & $\mathrm{L}$ & $\mathrm{L} / \mathrm{H}$ \\
\hline Étendue & $06.75-26.15$ & $13.75-43.26$ & $1.08-2.98$ \\
\hline Moyenne & $17.12-17.94$ & $26.43-28.03$ & $1.49-1.61$ \\
\hline
\end{tabular}

Espèce: Hemiaster (Mecaster) heberti (COQUAND, 1862)

Fig. 7.9 .

1862 Epiaster heberti COQUAND, p. 242, pl. 25, figs. 7-9.

1921 Hemiaster heberti mutatio Turonensis FOURTAU, p. 89, pl. 11, figs. 1-10.

2004a Hemiaster (Mecaster) heberti COQUAND turonensis FOURTAU - ABDEL-GAWAD et al., pl. 10, fig. 14a-b.

2006 Hemiaster (Mecaster) heberti (COQUAND) turonensis FOURTAU - El QOT, p. 153, pl. 34, fig. $6 a-b$.

2007 Hemiaster (Mecaster) heberti (COQUAND) turonensis FOURTAU - ABDEL-GAWAD et al., pl. 6, fig. 11.

2011 Hemiaster (Mecaster) heberti COQUAND AYOUB-HANNAA, p. 274, pl. 34, figs. 7-8.

Description: Espèce de petite à grande taille, polygonale. Sa hauteur maximale représente moins des deux tiers du diamètre postérieur. Surface apicale convexe. Surface adorale légèrement convexe avec légère dépression autour du péristome. Zone porifère étroite. Pores obliques, ovés en forme de fentes égales.

Discussion: Malgré la grande ressemblance, Hemiaster heberti diffère de Hemiaster latigrunda (Péron et Gauthier, 1880) en ayant des pétales appareillés un peu plus profonds et ambulacre frontal. La dernière espèce ne montre pas de marge antérieure profondément excavée.
Répartition: Cénomanien: Algérie (Coquand, 1862). Turonien: Égypte (Abdelhamid \& El Qot, 2001; El Qot, 2010; Abdel-Gawad et al., 2007; Ayoub-Hannaa, 2011).

Matériel: 4 spécimens trouvés dans l'unité D.

Espèce: Hemiaster (Mecaster) pseudofourneli (PÉRON \& GAUTHIER, 1878) Fig. 7.10.

1878 Hemiaster pseudofourneli sp. nov. PÉRON \& GAUTHIER in COTTEAU et al., p. 113, pl. 4, figs. 5-8.

1914 Hemiaster pseudofourneli PÉRON \& GAUTHIER - FOURTAU, p. 82, 109.

1963 Hemiaster pseudofourneli PÉRON \& GAUTHIER - FAWZI, p. 14.

1995 Mecaster pseudofourneli PÉRON \& GAUTHIER - NÉRAUDEAU, p. 418, fig. 6 d-e; text-fig. 7.

2002 Mecaster pseudofourneli PÉRON \& GAUTHIER - AHMAD \& ABDELHAMID, p. 459 , figs. 5. 1-4, 6, 8 .

2011 Mecaster pseudofourneli PÉRON \& GAUTHIER - AYOUB-HANNAA, p. 273, pl. 34 , fig. 6 .

Description: Espèce de taille moyenne, sub-hexagonale à ovale. Surfaces apicale et adorale convexes. Zone porifère petite et étroite. Pores égaux, obliques et non conjugués.

Discussion: Hemiaster pseudofourneli diffère de Hemiaster newtoni en ayant plus de pétales plus étroits et de taille égale. Hemiaster cubicus (DESOR, 1847) est caractérisé par un péristome extrêmement excentrique très proche de la marge antérieure, d'un petit périprocte et des sulcatures antérieures profondes.

Répartition: Cénomanien: Algérie (Péron \& Gauthier, 1878), Égypte (Fawzi, 1963; AyoubHannaa, 2011), Jordanie (Ahmad \& Al-Hammad, 2002) et Arabie Saoudite (Néraudeau, 1995).

Matériel: 2 spécimens trouvés dans l'unité A.

Espèce: Hemiaster batnensis (COQUAND, 1862) Fig. 7.11.

1862 Hemiaster batnensis COQUAND, p. 248, pl. 26, figs. 6-8.

1914 Hemiaster batnensis COQUAND -

FOURTAU, p. 66. 
1991 Mecaster batnensis COQUAND - SMITH \& BENGSTON, p. 56-60, figs. 46-47, 48a, 49; pls. 12-13. 2012 Mecaster batnensis COQUAND BENYOUCEF et al., pl. 2, fig. 7a-b. 2013 Mecaster batnensis COQUAND - RONEY, p. 11, 12, fig. 5.1-14.

Description: Coquille arrondie polygonale, à angles très obtus sur les cotés. Elle est coupée verticalement en arrière et légèrement sinueuse à l'aire anale. Un dessus assez plat et un dessous convexe, avec des dépressions ambulacaires peu marquées.

Discussion: Cette espèce montre des ressemblances avec Priaster fourneli et surtout avec Hemiaster verneuilli. Elle diffère du premier par l'absence de sillon latéral distinctif du genre, sa forme plus aplatie, ses ambulacres beaucoup moins creusés et par les sinuosités antérieure et postérieure bien moins concaves; et duu second par sa plus grande taille, son aire anale plus sinueuse et moins élevée, son périprocte toujours acuminé et enfin par sa largeur inférieure à sa longueur.

Répartition: Cénomanien: Brésil et Texas (Smith \& Bengtson, 1991), Egypte (El Qot, 2006) et Algérie (Coquand, 1862; Busson et al., 1999; Benyoucef et al., 2012).

Matériel: 5 spécimens trouvés dans l'unité A.

\section{Conclusion}

La présente étude comporte de nombreux éléments inédits à la connaissance de la macrofaune du Cénomanien sur un secteur-clé de la marge africaine de la Téthys, en l'occurrence, le Djebel Metrassi (Batna). L'étude taxonomique fait état d'un corail, de 26 espèces de bivalves ( 8 ordres, 15 familles), de 15 espèces de gastéropodes ( 5 ordres, 10 familles et 13 genres), de 3 espèces d'ammonites et de 6espèces d'échinides ( 3 ordres et 3 familles). Elle permet l'établissement d'une subdivision de la série cénomanienne et révèle d'étroites similitudes avec les taxons récoltés au sud de la Téthys. L'homogénéité de la faune cénomanienne et sa large répartition géographique en font un marqueur à affinité paléo-biogéographique permettant des corrélations précises pour les régions nordafricaines. Elle témoigne, également, de la stabilité environnementale relative des conditions paléoécologiques au cours du Cénomanien. Elle est significative de dépôts de mer peu profonde, bien oxygénée et avec un hydrodynamisme moyen à calme sur un substrat vaseux. Ainsi, une sédimentation de type plate-forme carbonatée s'installe dans les monts de Bellezma durant le Cénomanien, avec une faune de macro-invertébrés abondante et diversifiée.

\section{REMERCIEMENTS}

Les auteurs remercient vivement M. Makhloufi Ayoub pour son aide et son assistance sur le terrain. Leur gratitude va également aux deux rapporteurs anonymes qui ont contribué, par leurs remarques et leurs commentaires constructifs, à l'amélioration du manuscrit préliminaire.

\section{References}

Abbass, H.L. (1962). A monograph on the Egyptian Cretaceous pelecypods. Monographs of the Geological Museum, Palaeontological Series, United Arab Republic Geological Survey and Mineral Research Department, 224 pp.

Abdel-Gawad, G.I. \& Gameil, M. (1995). Cretaceous and Palaeocene coral faunas in Egypt and Greece. Coral Research Bulletin, 4: 1-36.

Abdel-Gawad, G.I. \& Gameil, M. (2002). Bivalvia taxonomy of the Cenomanian-Turonian strata of Gabal Nezzazat, west central Sinai, Egypt. Journal of the Faculty of Science of the United Arab Emirates, 124: 70-107.

Abdel-Gawad, G.I.; El-Sheikh, H.A.; Abdelhamid, M.A.; El-Beshtawy, M.K.; Abed, M.M.; Fürsich, F.T. \& El-Qot, G.M. (2004a). Stratigraphic studies on some Upper Cretaceous successions in Sinai, Egypt. Egyptian Journal of Paleontology, 4: 263-303.

Abdel-Gawad, G.I.; Orabi, O.H. \& Ayoub, W.S. (2004b). Macrofauna and biostratigraphy of the Cretaceous section of Gebel El-Fallig area, northwest Sinai, Egypt. Egyptian Journal of Paleontology, 4: 305-333.

Abdel-Gawad, G.I.; El-Qot, G.M. \& Mekawy, M.S. (2007). Macrobiostratigraphy of the Upper Cretaceous succession from southern Galala, Eastern Desert, Egypt. Second International Conference on the Geology of the Tethys, Cairo University, Cairo, 329-349.

Abdelhamid, M.A.M. \& El Qot, G.M. (2001). Upper Cretaceous echinoids from Gebel Hamra and El Minsherah, North Sinai, Egypt. Middle East Research Center, Earth science Series, 15: 1-31.

Abdelhamid, M.A.M. \& Azab, M.M. (2003). AptianCenomanian echinoids from Egypt. Revue de Paléobiologie, 22 (2): 851-876. 
Adams, H. \& Adams, A. (1853-1858). The Genera of Recent Mollusca; arranged to their organization. John Van Voorst, London, vol. 1, 484 pp.; vol. 2, 661 pp.; vol. 3, 136 pp.

Adkins, W.S. (1928). Handbook of Texas Cretaceous fossils. University of Texas Bulletin, 2838: 5-385.

Agassiz, L. \& Desor, E. (1846-1847). Catalogue raisonné des familles des genres et des espèces de la classe des Echinodermes. Annales des Sciences Naturelles, Zoologie, 3: 6-8.

Ahmad, F. \& Al-Hammad, A. (2002). Oysters and echinoids from the Naur Formation (Late Aptian- Early Cenomanian) of Central Jordan. Neues Jahrbuch für Geologie und Paläontologie, Monatshefte, 2002: 449-464.

Albanesi, C. \& Busson, G. (1974). Gastéropodes du Crétacé Supérieur de l'extrème-sud tunisien et de la région du Tinrhert (Sahara Algérien). Rivista Italiana di Paleontologia e Stratigrafia, 80: 251-342.

Aly, M.F.; Saber, S.G.; Abdel-Gawad, G.I. \& Ferieg, Y.F. (2005). Cenomanian-Turonian rudist buildups of northern Sinai, Egypt. Egyptian Journal of Paleontology, 5: 253-286.

Aly, M.F.; Smadi, A. \& Abu Azzam, H. (2008). Late Cenomanian-Early Turonian ammonites of Jordan. Revue de Paléobiologie, 27 (1): 43-71.

Alloiteau, J. (1952). Madréporaires post-paléozoiques. In: Traité de Paléontologie vol. 1 (Piveteau, J., Ed.), Masson et Cie., Paris, 539-684.

Amard, B.; Collignon, M. \& Roman, J. (1981). Etude stratigraphique et paléontologique du Crétacé Supérieur et Paléocène du Tinhert-W et Tademait-E (Sahara Algérien). Documents des Laboratoires de Géologie de la Faculté des Sciences de Lyon, Hors Série, 6, 15-173.

Amédro, F.; Matrion, B.; Touch, R. \& Verrier, J.M. (2012). Extension d'un niveau repère riche en Inoceramus crippsi [bivalve] dans le Cénomanien basal du Bassin Anglo-Parisien. Annales de la Société géologique du Nord, 19: 9-23.

Amédro, F. \& Robaszynski, F. (2010). La transgression cénomanienne et l'âge des Tourtias, du Nord du Bassin de Paris (F) et du Bassin de Mons (B). Bulletin d'Information des Géologues du Bassin de Paris, 47: 7-35.

Amler, M., Fischer, R. \& Rogalla, N. (2000). Muscheln. Enke-Verlag, Stuttgart, 214 pp.

Andreu, B (1989). Le Crétacé moyen de la transversale Agadir-Nador (Maroc): Précisions stratigraphiques et sédimentologiques. Cretaceous Research, 10: 49-80. https://doi.org/10.1016/0195-6671(89)90029-3

Aqrabawi, M. (1993). Oysters (Bivalvia-Pteriomorphia) of the Upper Cretaceous rocks of Jordan. Palaeontology, stratigraphy and comparison with the Upper Cretaceous oysters of Northwest Europe - Mitteilungen aus dem Geologisch-Paläontologischen Institut der Universität Hamburg, 75: 1-135.
Ayoub-Hannaa, W.S. (2011). Taxonomy and Palaeoecology of the Cenomanian-Turonian Macro-Invertebrates from Eastern Sinai, Egypt. Thèse de Doctorat, Université de Würzburg, 386 pp.

Ayoub-Hannaa, W.S. \& Fürsich, F.T. (2011). Functional morphology and taphonomy of Cenomanian (Cretaceous) oysters from the eastern Sinai Peninsula, Egypt. Palaeobiodiversity and Palaeoenvironments, 91: 197-214. https://doi.org/10.1007/ s12549-011-0051-7

Ayoub-Hannaa, W.S.; Fürsich, F.T. \& El-Qot, G.M. (2014). Cenomanian-Turonian bivalves from eastern Sinai, Egypt. Palaeontographica, A301: 63-168. https://doi.org/10.1127/pala/301/2014/63

Ayoub-Hannaa, W.S.; Radulovic, B.; Radulovic, V.J. \& Fursich, F. (2015). Gastropods from the Lower Cenomanian of Koracica (Kosjman Mountain, central Serbia). Neues Jahrbuch für Geologie und Paläontologie, Abhandlungen, 376 (1): 27-62. https:// doi.org/10.1127/njgpa/2015/0474

Bandel, K. \& Geys, J.F. (1985). Regular echinoids in the Upper Cretaceous of the Hashemite Kingdom of Jordan. Annales de la Société Géologique du Nord, 104: 97-115.

Barber, W. (1958). Upper Cretaceous Mollusca from north-eastern Nigeria. Records of the Geological Survey of Nigeria Bulletin, 1956: 14-46.

Baroni, C.; Incitti, L.; Oliveri, A. \& Viola, V. (1953). Revisione della fauna neocretacica della Libia: Pinnidae, Mytilidae, Limidae, Nuculidae, Ledidae, Arcidae, Cyprinidae, Astartidae, Crassatellidae, Veneridae, Cardiidae, Lucinidae, Aloididae, Thraciidae, Chamidae. Annali del Museo Libico di Storia Naturale, 4: 11-110.

Baron-Szabo, R.C. (2002). Scleractinian corals of the Cretaceous. Knoxville, USA, 539 pp.

Bayle, E. (1878). Explication de la carte géologique de la France, Atlas, Fossiles principaux des terrains. Tome 2. Imprimerie Nationale, Paris.

Benkherouf, F. (1988). Les foraminifères cénomaniens des Alpes-Maritimes (Sud-est de la France) et du djebel Dyr (NE Algérie): biostratigraphie et paléo-environnement. Thèse de Doctorat, Université de Nice.

Bellion, Y. (1972). Etude géologique et hydrogéologique de la partie occidentale des Monts de Bellezma. Thèse 3ème cycle, Université de Paris VI, 221 pp.

Benyoucef, M.; Bensalah, M.; Malti, F.Z. \& Bendella, M. (2008). New stratigraphic data from the cretaceous basin of Guir (Béchar, South-western of Algeria). Documents des Laboratoires de Géologie, 164: 19-22.

Benyoucef, M.; Meister, C.; Bensalah, M. \& Malti, F. (2012). La plate-forme préafricaine (Cénomanien supérieur-Turonien inferieur) dans la région de Bechar (Algérie): Stratigraphie, paléoenvironnement et signification palébiogéographique. Revue de Paléobiologie, 31 (1): 205-218. 
Benyoucef, M. \& Meister, C. (2015). Lithostratigraphic evolution, facies analysis and depositional environment of the Cenomanian-lower Turonian in the Guir area, southwestern Algeria. Cretaceous Research, 53: 68-88. https://doi.org/10.1016/j.cretres.2014.10.009

Benyoucef, M.; Meister, C.; Mebarki, K.; Läng, E.; Adaci, M.; Cavin, L.; Malti, F.Z.; Zaoui, D.; Cherif, A. \& Bensalah, M. (2016). Evolution lithostratigraphique, paléo-environnementale et séquentielle du Cénomanien-Turonien inférieur dans la région du Guir (Ouest algérien). Carnets de Géolologie, 16(9): 271295. https://doi.org/10.4267/2042/59926

Benyoucef, M.; Mebarki, K.; Ferré, B.; Adaci, M.; Bulot, L.G.; Desmares, D.; Villier, L.; Bensalah, M.; Frau, C.; Ifrim, C. \& Malti, F. (2017). Litho- and biostratigraphy, facies patterns and depositional sequences of the Cenomanian-Turonian deposits in the Ksour Mountains (Saharan Atlas, Algeria). Cretaceous Research, 78: 34-55. https://doi.org/10.1016/j.cretres. 2017.05.013

Berndt, R. (2002). Palaeoecology and taxonomy of the macrobenthic fauna from the Upper Cretaceous Ajlun Group, southern Jordan. Thèse de Doctorat, Université de Würzburg, $221 \mathrm{pp}$.

Berndt, R. (2003). Cenomanian echinoids from Southern Jordan. Neues Jahrbuch für Geologie und Paläontologie, Monatshefte, 2: 73-90.

Blanckenhorn, M. (1890). Beiträge zur Geologie Syriens: Die Entwickelung des Kreidesystems in Mittel und Nord-Syrien, mit besonderer Berück sichtigung der paläontologischenVerhältnisse nebst einem Anhang über den jurassischen Glanda rienkalk. Selbsetverlag des Verfassers, Cassel, 135 pp.

Blanckenhorn, M. (1927). Die fossilen Gastropoden und Scaphopoden der Kreide von Syrien-Palästina. Palaeontographica, 69: 111-186.

Blanckenhorn, M. (1934). Die Bivalven der Kreideformation von Syrien-Palästinan ebsteinemer gänzenden Anlangüber Brachiopoden, Gastropoden und Pteropoden und einem Überblicküber die gesamte Molluskenfauna. Palaeontographica, A81: 161-296.

Boreham, A.U.E. (1959). Cretaceous fossils from the Chatham Islands. Transactions of the Royal Society of New Zealand, 86: 119-125.

Bouchet, P. \& Rocroi, J.P. (2005). Classification nomenclator of gastropod families. Malacologia 47: 1-397.

Brongniart, A. (1822). Sur quelques terrains de Craie hors du Bassin de Paris. In: Description géologique des environs de Paris (Cuvier, G. \& A. Brongniart, A., Eds.), Paris, 428 pp.

Bureau, D. (1967). Touggourt (Monts du Belezma). Compte rendu de la Société géologique française. 19 juin 1967: 237-239.

Bureau, D. (1975). Esquisse géologique des Monts de Belezma-Aurès, Algérie. Publication du Service de Carte Algérie, Bulletin, 45: 75-92.
Bureau D. (1986). Approche sédimentaire de la dynamique structurale: Evolution mésozoïque et devenir orogénique de la partie septentrionale du fossé saharien (SudOuest constantinois et Aurès, Algérie). Thèse de Doctorat en Sciences, Université de Pierre et Marie Curie Paris, 441 pp.

Burollet, P.F. \& Desforges, G. (1982). Dynamique des bassins néocrétacés en Tunisie. Mémoire géologique de l’Université de Dijon, 7: 381-389.

Busson, G.; Dhondt, A.; Amédro, F.; Néraudeau, D. \& Cornée, A. (1999). La grande transgression du Cénomanien supérieur-Turonien inférieur sur la Hamada de Tinrhert (Sahara algérien): datations biostratigraphiques, environnement de dépôt et comparaison d'un témoin épicratonique avec les séries contemporaines à matiére organique du Maghreb. Cretaceous Research, 20: 29-46. https://doi.org/10.1006/cres.1998.0137

Buchbinder, B.; Benjamini, C. \& Lipson-Benitah, S. (2000). Sequence development of Late CenomanianTuronian carbonates ramps, platforms and basins in Israel. Cretaceous Research, 21: 813-843. https://doi. org/10.1006/cres.2000.0228

Carlsson, J.G. (1938). Malms samling av Kritfossil frän Kristianstadsomrâdet. I. Cephalopoda, Gastropoda, Lamellibranchiata, och Brachiopoda. Göteborgs kungl. vetenskapsoch vitterhets samhäles handlingar, (5), B $6, n^{\circ} 5: 1-25$. Gotemburgo.

Chikhi-Aouimeur, F. (1995). Caprinula aff. cedrorum (Blanckenhorn, 1890) from the Upper Cenomanian of western Algeria. Revista mexicana de Ciencias geológicas, 12: 179-184.

Chikhi-Aouimeur, F. (1998). Les Rudistes du Crétacé Supérieur de l'Algérie. Etude paléontologique. Données écologiques, biostratigraphiques et paléogéographiques. Thèse Doctorat es Sciences. Université de H. Boumediene, Alger. 198 pp.

Chikhi-Aouimeur, F. (2004). Description of some rudists collected by J. Savorninin the Cenomanian-Turonian of northeastern Algeria (Setif and Hodna region). In: Proceedings of the 5th International Congress on Rudists, Erlangen, Germany 1999 (R. Höfling, R., Ed.), Courier Forschungs institute Senckenberg, 247: 49-6.

Chikhi-Aouimeur, F.; Nouh Mefnoune, B. \& Bankih, K. (2006). Rudistes du Cénomanien et du Turonien de la région de Ghardaïa (Mzab, Algérie). Paléontologie, biostratigraphie et paléobiogéographie. Mémoire du Service Géologique National, Algérie, 13: 199-207.

Chikhi-Aouimeur, F. (2010). L'Algérie à travers son patrimoine paléontologique: les rudistes. BAOSEM, Alger, $270 \mathrm{pp}$.

Chiplonklar, G.W. \& Badve, R.M. (1972). PaleontoIogy of the Bagh beds; II, Echinoides. Proceedings of the national Academy of Sciences, India Section, B 76, (4): 138-139.

Choffat, P. (1886-1902). Recueil d'études paléontologiques sur la faune crétacique du Portugal. Section des travaux géologiques du Portugal, 4 vols., 171pp. 
Clark, H.L. (1917). Hawaiian and other Pacific Echinids. Memoirs of the Museum of Comparative Zoology. 46/2: 85-283.

Claus, C.F.W. (1876). Grundzüge der Zoologie. Elwertsche Universitätsbuchhandlung. 3ème ed. Marburg, Leipzig, 1254 pp.

Collignon, M. (1971). Gastéropodes et Lamellibranches du Sahara. Annales de Paléontologie (Invertébrés), 57: 143-202.

Conrad, T.A. (1852). Description of the fossils of Syria, collected in the Palestine expedition. In: Official report of the United States expedition to explore the Dead Sea and the River Jordan (Lynch, W.F., Ed.), Murphy and Co., Baltimore, 235 pp.

Conrad, T.A. (1870), Notes on Recent and fossil shells, with descriptions of new species: American Journal of Conchology, 6 (1): 71-78.

Coquand, M.H. (1862). Géologie et Paléontologie de la région sud de la Province de Constantine. Memoires de la Société d'Emulation de la Provence, 2: 1-341.

Coquand, M.H. (1869). Monographie du genre "Ostrea". Terrain crétacé, $215 \mathrm{pp}$.

Coquand, M.H. (1880). Etudes supplémentaires sur la paléontologie algerienne faisant à la déscription géologique et paléontologique de la region sud de la province de Constantine. Bulletin de l'Académie d'Hippone, 15: 1-451.

Cossmann, M. \& Peyrot, A. (1919). Conchologie Néogénique de l'Aquitaine. Actes de la Société Linnéenne de Bordeaux, 70: 181-491.

Cotteau, G. \& Triger, J. (1855-1869). Echinides du département de la Sarthe. Bailliere et fils, Paris, $458 \mathrm{pp}$.

Cotteau, G.; Péron, P. \& Gauthier, V. (1876-1881). Echinides fossiles de l'Algérie. 1: pt. 3 (1876), Etage Urgo-Aptien et al bien: 1-90; pt. 4 (1878), Etage Cénomanien: 1-144; pt. 5 (1879), Etage Cenomanien: 145-234; 2: pt. 6 (1879), Etage Turonien: 1-110; pt. 7 (1881), Etage Sénonien: 1-118, G. Masson, Paris.

Cotteau, G.; Péron, A. \& Gauthier, V. (1885). Echinides fossiles de l'Algérie, 9 ème fasc. Etage éocène. G. Masson, Paris, 89 pp.

Cox, L.R. (1929). A synopsis of the Lamellibranchia and Gastropoda of the Portland Beds of England. Part 1, Lamellibranchia. Proceedings of the Dorset Natural History and Archaeology Society, 50: 131-202.

Dall, W.H. (1889). On the hinge of pelecypods and its development, with an attempt toward a better subdivision of the groups: American Journal of Science, 38(3): 445-462. https://doi.org/10.2475/ajs.s3-38.228.445

Da Costa, E.M. (1778). Historia naturalis Testaceorum Britanniae or The British Conchology. London (Millan, White, Elmsley \& Robson), 254 pp.

Dartevelle, E. \& Brévion, P. (1956). Mollusques fossiles du Crétacé de la côte occidentale d'Afrique du Cameroun à l'Angola. I. Gastéropodes. Annales du Musée Royal Congo Belge, Sciences géologiques., 15: 1-128.
Dartevelle, E. \& Freneix, S. (1957). Mollusques fossiles du Crétacé de la côte occidentale d'Afrique du Cameroun à l'Angola. II. Lamellibranches. Annales du Musée Royal du Congo Belge, 20: 1-271.

De Férussac, A.E. (1822). Tableaux systématiques des animaux mollusques. A. Bertrand, Paris, $111 \mathrm{pp}$.

Defrance, M.J.L. (1825). Minéralogie et géologie. In: Dictionnaire des Sciences Naturelles, Tome 35. F.G. Levrault, Eds., Paris, 210 pp.

Delamette, M. \& Kennedy, W.J. (1991). Cenomanian ammonites from the condensed deposits of the Helvetic Domain. Journal of Paleontology, 65: 435465. https://doi.org/10.1017/S0022336000030407

Delpey, G. (1939). Les Gastéropodes Mésozoiques de la Région Libanaise. Notes et Mémoires de la Section d'Études géologiques du Haut-Commissariat de la République Française en Syrie et au Liban, 292 pp.

Dhondt, A.V. (1973). Systematic revision of the subfamily Neitheinae (Pectinidae, Bivalvia, Mollusca) of the European Cretaceous. Mémoire de 1'Institut Royal des Sciences Naturelles de Belgique, 176: 5-101.

Dhondt, A.V. (1982). Some Spanish Cretaceous bivalves. Cuadernos de Geologia Ibérica, 8: 847-865.

Dhondt, A.V. \& Dieni, I. (1993). Non-rudistid bivalves from Late Cretaceous rudist limestones of NE Italy (Col dei Schiosi and Lago di S. Croce areas). Memorie Scienze Geologiche, 45: 165-241.

Dhondt, A.V. \& Jaillard, E. (2005). Cretaceous bivalves from Ecuador and Northern Peru. Journal of South American Earth Sciences, 19: 325-342. https://doi. org/10.1016/j.jsames.2005.01.005

D’Hombres-Firmas, L.A.D. (1838). Recueil de mémoires et d'observations de physique, de météorologie, d'agriculture et d'histoire naturelle. Ballivet et Fabre, Nimes: 278 pp.

D’Orbigny, A.D.E. (1840-1842). Paléontologie française, description zoologique et géologique de tous les animaux mollusques et rayonnés de France. Terrains Crétacés. Vol. 1. Céphalopodes. Masson ed., Paris: $662 \mathrm{pp}$

D’Orbigny, A.D.E. (1843). Considérations sur l'ensemble des Mollusques Gastéropodes des terrains crétacés. Annales des Sciences Naturelles, sér. 2, t. 20, p. $26-54$

D’Orbigny, A.D.E. (1847). Considération zoologiques et géologiques sur les Brachiopodes. Annales des Sciences Naturelles, 3: 1-259.

D’Orbigny, A.D.E. (1850). Prodrome de Paléontologie stratigraphique universelle des animaux Mollusques et rayonnés. Vol. 2, 427 p., G. Masson, Paris, 427 pp. https://doi.org/10.5962/bhl.title.62810

Douvillé, M.H. (1904). Paléontologie, mollusques fossiles. In: Mission Scientifique en Perse (J. de Morgan, Ed.), Vol. 3, pt. 4, Leroux, Paris, 191-380.

Douvillé, M.H. (1913). Description des rudistes de l'Egypte. Mémoires présentés à l'Institut Egyptien, 6: $237-256$. 
Douvillé, M.H. (1916). Les terrains secondaires dans le massif du Moghara, à l'est de l'isthme de Sues, d'après les explorations de Couyat-Barthoux. Mémoires de l'Académie des Sciences de l'Institut de France, 55: 1-184.

Drouet, C. (1824). Sur un nouveau genre de coquille de la famille des Arcacées et description d'une nouvelle espèce de Modiole fossile: Mémoires de la Société linnéenne de Paris, 3: 183-192.

Dubourdieu, G. \& Durozoy, G. (1950). Observations tectoniques dans les environs de Tébessa et de l'Ouenza. Bulletin de la Société géologique de France, 20: 257-26.

Duncan, P.M. (1889). A revision of the genera and great groups of the Echinoidea. Journal of the Linnean Society (Zoology.), 23: 1-311. https://doi. org/10.1111/j.1096-3642.1889.tb01431.x

Eck, O. (1914). Die Cephalopoden der Schweinfurthschen Sammlung aus der Oberen Kreide Ägyptens. Zeitschrift der Deutschen Geologischen Gesellschaft, 66: 179-216.

El Qot, G.M. (2006). Late Cretaceous macrofossils from Sinai, Egypt. Beringeria, 36: 3-163.

El Qot, G.M. (2010). Upper Cretaceous echinoids from Galala Plateaux, North Eastern Desert, Egypt. Revue de Paléobiologie, 29 (1): 261-291.

Ettachfini, E.M. \& Andreu, B. (2004). Le Cénomanien et le Turonien de la Plate-forme Préafricaine du Maroc, Cretaceous Research, 25 (2): 277-302. https://doi. org/10.1016/j.cretres.2004.01.001

Faujas de Saint-Fond, B. (1799). Histoire naturelle de la Montagne de Saint-Pierre de Maestricht. H. J. Jansen, Paris, 263 pp.

Fawzi, M.A. (1963). La faune Cènomanienne d'Egypte. Geological Survey of Egypt, Monograph, 2: 1-133.

Felix, J. (1914). Anthozoa Cretacea. Fossilium Catalogus, (1: Animalia) 5-7, W. Junk, Berlin, 273 pp.

Ficheur, E. (1901). Notice sommaire sur la carte géologique de l'Algérie, 3ème édition. Congrés International. Paris, 14 pp.

Fischer, P. (1880-1887). Manuel de Conchyoliologique. G. Masson, Paris, 1369 pp.

Fleming, J.D.D. (1828). A History of British Animals, exhibiting the descriptive characters and systematical arrangement of the genera and species of Quadrupeds, Birds, Reptiles, Fishes, Mollusca, and Radiata of the United Kingdom. Bell and Bradfute, Edinburgh, 565 pp.

Fluteau, F.; Ramtein, G.; Besse, J.; Guiraud, R. \& Masse, J.P. (2007). Impacts of paleo-geography and sea level on mid-cretaceous. Palaeogeography, Palaeoclimatology, Palaeoecology, 247: 357-381. https://doi. org/10.1016/j.palaeo.2006.11.016

Forbes, E. (1846). Report on the fossil Invertebrata from southern India, collected by Mr. Kate \& Mr. Cunliffe. Transactions of the Geological Society of London, (2)7: 97-174.
Fourtau, R. (1904). Contribution à l'étude de la faune Crétacique d'Egypte. Bulletin de l'Institut Egyptien, 4: 231-249.

Fourtau, R. (1914). Catalogue des invertébrés fossiles de l'Egypte. Terrains Crètacès, 1, Èchinodermes. Geological Survey of Egypt, Palaeontological Series, 2: 1-109.

Fourtau, R. (1917). Catalogue des Invertébrés fossiles de l'Egypte repésentés dans les collections du Musée de Géologie au Caire. Terrains Crétacés 2me Partie: Mollusques Lamellibranches. Geological Survey of Egypt, Palaeontological Series, 3:1-108.

Fourtau, R. (1921). Catalogue des invertébrés fossiles de l'Egypte. Terrains Crétacés, 3, Èchinoderms (Supplément). Geological Survey of Egypt, Palaeontological Series, 5: 1-101.

Freneix, S. (1972). Les Mollusques bivalves crétacés du bassin côtier de Tarfaya (Maroc méridional). Notes et Mémoires du Service Géologique du Maroc, 228: 49-255.

Gabb, W.M. (1869). Cretaceous and Tertiary fossils: California Geological Survey, Paleontology, 2: 1-299.

Gertsch, B.; Keller, G.; Adatte, T.; Berner, Z.; Kassab, A.S.; Tantawy, A.A.A.; El-Sabbagh, A.M. \& Stueben, D. (2008). Cenomanian-Turonian transition in a shallow water sequence of the Sinai, Egypt. International Journal of Earth Sciences, 1-18.

Geys, J.F. (1989). Hemicidaroid echinoids from Upper Cretaceous deposits in Wadi Qena area (Eastern desert, Egypt). Bulletin de l'Institut Royal des Science Naturelles de Belgique, 59: 127-135.

Gill, G.A. \& Lafuste, J. (1987). Structure, répartition et signification paléogéographique d'Aspidiscus, hexacoralliaire cénomanien de la Téthys. Bulletin de la Société géologique de France, 3: 921-934.

Gill, G.A. \& Chikhi, F. (1991). Remarks on new occurrences of Aspidiscus, a Cenomanian scleractinian coral in the Persian Gulf and in Algeria. Lethaia, 24: 349-350. https://doi.org/10.1111/j.1502-3931.1991. tb01485.x

Glaçon, G. (1952). Les Monts de Hodna (partie orientale). Publication du 19e Congrès International de Géologie, Alger, Monographie Régionale, 10: $92 \mathrm{pp}$.

Goldfuss, A. (1833). Petrefacta Germaniae, Vol. 2. Arnz und Co., Düsseldorf, 68 pp.

Gray, J.E. (1824). A supplement to the appendix of Captain Perry's voyage for the discovery of a North West Passage, in the Years 1819-1820, containing an account of the subjects of Natural History. In: Journal of a Second Voyage for the Discovery of a Northwest Passage from the Atlantic to the Pacific, Performed in the Years 1821-22-23 (W. E. Parry, ed.), Appendix 10, Zoology, John Murray, London, 240-246.

Gray, J.E. (1848). On a peculiar structure in shells with some observations on the shell of Sphaerulites. Magazine of Zoology and Botany, 2: 228-232. 
Gray, J.E. (1850). Figures of molluscous animals selected from various authors. Vol. 4. Longman, Brown, Green, Longmans, and Roberts, London, 219 pp.

Greco, B. (1916). Fauna cretacea dell'Egitto raccolta dal Figari Bey. Parte seconda. Gastropoda. Palaeontographica Italica, Memorie di Palaeontologia, 22: 103-170.

Greco, B. (1918). Fauna cretacea dell'Egitto raccolta dal Figari Bey Parte terza: Lamellibranchiata (cont. e fine). Fasc. 2- Lamellibranchi del Turoniano e del Cenomaniano. Palaeontographica Italica, Memorie di Palaeontologia, 24: 1-58.

Grossouvre, A. (1894). Recherches sur la Craie supérieure, 2, Paléontologie. Les ammonites de la Craie supérieure. Mémoires du Service de la Carte Géologique détaillée de la France: 1-264.

Grundel, L. (1982). Ostreen (Bivalvia) aus der Sächsischen Oberkreide. II. Abhandlungen des Staatlichen Museums fur Mineralogie und Geologie zu Dresden, Leipzig, 31: 151-160.

Guiraud, R. (1973). Evolution post-triasique de l'avant pays de la chaine alpine en Algérie, d'après l'étude du bassin du Hodna et des régions voisines. Doctorat ès Sciences, Université de Nice, 270 pp.

Hamlin, C.E. (1884). Results of an examination of Syrian Molluscan fossils, chiefly from the Range of Mount Lebanon. Memoirs of the Museum of Comparative Zoology, 10: 1-68. https://doi.org/10.5962/bhl. title. 48422

Hardouin, L. (1868). Sur la géologie de la subdivision de Constantine. Bulletin de la Société géologique de France, 25: 1-328.

Herkat, M. (2007). Application of correspondance analysis to paleobathymetric reconstitution of Cenomanian and Turonian (Cretaceous) rocks of Eastern Algeria. Paleogeography, Paleoclimatology, Paleoecology, 254 (3): 583-605. https://doi.org/10.1016/j. palaeo.2007.07.011

Kassab, A.S. \& Zakhera, M.S. (2002). Upper Cretaceous oysters from the northern part of the Eastern Desert, Egypt. Neues Jahrbuch für Geologie und Paläontologie, Abhandlungen, 224: 1-30.

Kazi-Tani, N. (1986). Evolution géodynamique du segment alpin d'Algérie. 70e R.A.S.T., Bordeaux, 315 pp.

Kennedy, W.J. \& Gale, A.S. (2015). Upper Albian and Cenomanian ammonites from Djebel Mrhila, Central Tunisia. Revue de Paléobiologie, Genève, 34(2): 235-361.

Kennedy, W.J.; Klinger, H.C. \& Lehmann, J. (2015). Cretaceous faunas from Zululand and Natal, South Africa. The ammonite Subfamily Mantelliceratinae Hyatt, 1903. African Natural History, Cape Town, 11: 1-42.

Kennedy, W.J.; Amédro, F.; Robaszynski, F. \& Jagt, J.W.M. (2011). Ammonite faunas from condensed Cenomanian-Turonian sections ('Tourtias') in southern Belgium and northern France. Netherlands Journal of Geoscience, 90: 209-238. https://doi.org/10.1017/ S0016774600001128
Kennedy, W.J. \& Juignet, P. (1981). Upper Cenomanian ammonites from the environs of Saumur, and the provenance of the types of Ammonites vibrayeanus and Ammonites geslinianus. Cretaceous Research, 2: 19-49. https://doi.org/10.1016/S0195-6671(81) 80003-1

Khalil, H. \& Mashaly, S. (2004). Stratigraphy and stage boundaries of the Upper Cretaceous, Lower Paleogene succession in Gabal Musaba Salama area, southwestern Sinai, Egypt. Egyptian Journal of Paleontology, 4: 1-38

Kollmann, H.A. (1985). Upper Cretaceous gastropods from excavations for the highways A10 (Charente, France). Cretaceous Research, 6: 85-111. https://doi. org/10.1016/0195-6671(85)90030-8

König, C.D.E. (1825). Icones fossilium sectiles. G. B. Sowerby, London, 23pp. https://doi.org/10.5962/bhl. title. 60262

Kora, M.; Khalil, H. \& Sobhy, M. (2001). CenomanianTuronian macrofauna from the Gulf of Suez region: biostratigraphy and paleobiogeography. Egyptian Journal of Geology, 45: 441-462.

Kroh, A. \& Smith, A.B. (2010). Classification and phylogeny of post-Paleozoic echinoids. Journal of Systematic Palaeontology, 7: 147-212. https://doi. org/10.1080/14772011003603556

Laffitte, R. (1939). Etude géologique de l'Aurès. Bulletin du Service de Carte géologique de l'Algérie, 2éme série, $15.484 \mathrm{pp}$.

Lamarck, J.B.P. (1806). Sur les fossils des environs de Paris. Annales du Musée d'Histoire Naturelle: 156-166.

Lamarck, J.B.P. (1799). Prodome d'une nouvelle classification des coquilles. Mémoires de la Société d'Historie Naturelle de Paris, 1: 63-91.

Lamarck, J.B.P. (1801). Système des animaux sans vertèbres. Deterville, Paris, 432 pp.

Lamarck, J.B.P. (1819). Histoire naturelle des animaux sans vertebres. Suite des conchiferes. Chez L'Auteur, Paris, $258 \mathrm{pp}$.

Lambert, J. (1900). Etude sur quelques échinides de l'Infra-Lias et du Lias. Bulletin de la Société des Sciences Historiques Naturelles de l'Yonne, 52 (1899): 3-57.

Lambert, J. (1931-1932). Etude sur les echinides fossiles du nord de l'Afrique. Mémoires de la Société Géologique de France, 16: 1-228.

Lartet, L. (1873). Essai sur la Géologie de la Palestine et des contrées avoisinantes telles que l'Egypte et 1'Arabie. Deuxième Partie. Paléontologie. Annales de Science de Géologie (Pelecypods): 49-71.

Leach, W.E. (1819). Descriptions des nouvelles espèces d'animaux découvertes par le vasseau Isabelle dans un voyage au pôle boréal. Journal de Physique, de Chimie, d'Histoire Naturelle et des Arts, 88 (6): 462-467.

Linné, C. (1758). Systema naturae per regna tria naturae, secundum classes, ordines, genera, species, cum 
characteribus, differentiis, synonymis, locis. Editio decima, reformata. Tomus I. 824 pp. https://doi.org/ 10.5962/bhl.title.542

Linné, C. (1767). Systema Naturae, édition XIII de Gmelin.

Lovèn, S.L. (1847). MaIacozooIogy. Öfversigt af KongIiga Vetenskaps-Akademiens ForhandIingar, 175-199.

Lovèn, S.L. (1874). Etudes sur les echinoidées. Kongelige Svenska Vetenskaps Akademiens Handlingar, New Series, 11: 1-91.

Lundgren, B. (1894). Jämförelse mellan molluskfaunan i mammillatus och mucronata zonera i nordöstra Skâne (Kruistianstadsomrâdet). Kongelige svenska Vetenskaps-akademiens Handlingar, 26: 3-58.

Malchus, N. (1990). Revision der Kreide-Austern (Bivalvia-Pteriomorpha) Ägyptens (Biostratigraphie, Systematik). Berliner geowissen schaftliche Abhandlungen, A125: 1-231.

Masse, J.P.; Maresca, M.G. \& Sinni, E.L. (1998). Albian rudist faunas from southern Italy: taxonomic, biostratigraphic and palaeobiogeographic aspects. Geobios, 31: 47-59. https://doi.org/10.1016/ S0016-6995(98)80096-X

Masse, J.P.; Fenerci-Masse, M.; Vilas, L. \& Arias, C. (2007). Late Aptian-Albian primitive Radiolitidae (bivalves, hippuritoidea) from Spain and SW France. Cretaceous Research, 2: 697-718. https://doi. org/10.1016/j.cretres.2006.09.004

Meister, C. \& Rhalmi, M. (2002). Quelques ammonites du Cénomanien-Turonien de la région d'ErrachidiaBoudnid-Erfoud (partie méridionale du Haut Atlas Central, Maroc). Revue de Paléobiologie, 21 (2): 759-779.

Meister, C. \& Abdallah, H. (2005). Précision sur les successions du Cénomanien-Turonien dans la région de Gafsa, Tunisie du centre-sud. Revue de Paléobiologie, 24: 111-199.

Mekawy, M.S. (2007a). Gastropods of the CenomanianSantonian sequence from north Eastern Desert, Egypt. Egyptian Journal of Geology, 51: 149-176.

Mekawy, M.S. (2007b). Upper Cretaceous bivalves from Galala Plateaux, north Eastern Desert, Egypt: A systematic paleontology. Egyptian Journal of Paleontology, 7: 197-243.

Mekawy, M. S. \& Abu-Zied, R.H. (2008). Lower Cretaceous Molluscan fauna from north Sinai, Maghara area, Egypt. Egypt. Journal of Paleontology., 8: 291-334.

Mortensen, T. (1904). The Danish expedition to Siam, 1899-19900: II, Echinoidea. kongelige Danske Videnskabernes Selskabs skrifter, 7: 1-198.

Morycowa, E. \& Roniewicw, E. (1995). Microstructural disparity between Recent fungiine and Mesozoic microsolenine scleractinians. Acta Palaeontologica Polonica 40: 361-385.

Naili, H.; Belhadj, Z.; Robaszynski, F. \& Caron, M. (1995). Présence de roches mère à faciès Bahloul vers la limite Cénomanien-Turonien dans la région de Tébessa (Algérie orientale). Notes du service géologique de Tunisie, 61: 19-32.

Néraudeau, D. (1995). Diversité des échinides fossiles et reconstitutions paléo-environnementales. Geobios, Mémoire Spécial, 18: 337-345. https://doi.org/10.1016/ S0016-6995(95)80179-0

Néraudeau, D. \& Courville, P. (1997). Cenomanian and Turonian echinoids from Nigeria. Géobios 30: 835-847. https://doi.org/10.1016/S0016-6995(97) 80185-4

Néraudeau, D. \& Moreau, P. (1989). Paléoécologie et paléobiogéographie des faunes des échinides du Cénomanien nord-aquitain (Charente-Maritime, France). Géobios, 22(3): 293-324. https://doi.org/10.1016/ S0016-6995(89)80134-2

Neumayr, M. (1875). Die Ammoniten der Kreide und die Systematik der Ammonitiden. Zeitschrift der Deutschen Geologischen Gesellschaft, 27: 854-942.

Newell, N.D. \& Boyd, D.W. (1995). Pectinoid bivalves of the Permian-Triassic crisis. Bulletin of the American Museum of Natural History, 227: 1-95.

Nilsson, S. (1827). Petrificata Suecana formationis cretacea, descripta et inconibus illustrata. Pars prior, Vertebrata el Mollusca sistens. Officina Berlingiana., Londini Gothorum (Lund), 39 pp.

Noetling, F. (1897). Fauna of Baluchistan: The fauna of the Upper Cretaceous (Maastrichtian) beds of the Mari. Palaeontologica Indica, series 16, 1 (3): 1-79.

Özer, S. \& Ahmad, F. (2016). Caprinula and Sauvagesia rudist faunas (Bivalvia) from the Cenomanian of NW Jordan. Stratigraphy and taxonomy. Cretaceous Research, 58: 141-159. https://doi.org/10.1016/j. cretres.2015.05.002

Pandey, D.K.; Fursich, F.T. \& Heinze, M. (1996). Contributions to the Jurassic of Kachchh, Western India. V. The bivalve fauna. Part II. Subclass Anomalodesmata. Beringeria, 18: 51-87.

Pandey, D.K.; Fürsich, F.T.; Gameil, M. \& Ayoub-Hanna, W.S. (2011). Aspidiscus cristatus (Lamarck) from the cenomanian sediments of Wadi Quseib, east Sinai, Egypt. Journal of the Paleontological Society of India. 56 (1): 29-37.

Péron, A. (1877). Observations sur la faune des calcaires à Echinides de Rennes-les Bains et sur quelques fossiles du terrain Crétacé Supérieur. Bulletin de la Société Géologique de France, 3(5): 499-535.

Péron, A. (1889-1891). Déscription des mollusques fossiles des terrains crétacés de la région sud des Hauts-Plateaux de la Tunisie recueillis en 1885 et 1886 par Thomas, M. P. In: Exploration Scientifique de la Tunisie: $405 \mathrm{pp}$, Paris.

Pervinquière, L. (1907). Etudes de paléontologie tunisienne. I. Céphalopodes des terrains secondaires. Mémoires de la Carte Géologique de la Tunisie, F. -R- de Rudeval, Paris, 438 pp.

Pervinquière, L. (1912). Études de paléontologie tunisienne. II. Gastéropodes et Lamellibranches des 
terrains Crétacés. Mémoires de la Carte Géologique de la Tunisie, F. -R- de Rudeval, Paris, 352 pp.

Perrilliat, M.C.; Ahmad, F. \& Vega, F.J. (2006). Upper Cretaceous (Cenomanian-Turonian) bivalves from northern Jordan, Middle East. Revista Mexicana de Ciencias Geológicas, 23: 96-106.

Petitot, J.M. (1961). Contribution à l'étude des échinides fossiles du Maroc. Notes et Mémoires du Service Géologique du Maroc, 146: 1-183.

Philip, J.; Borgomano, J. \& Maskiry, S. (1995). Cenomanian Early Turonian carbonate platform of Northern Oman: Stratigraphy and palaeoenvironments. Cretaceous Research, 119: 77-92.

Philippi, R.A. (1853). Handbuch der Conchyliologie und Malacozologie. Anton, Halle, 547 pp. https://doi.org/ 10.5962/bhl.title. 10373

Pomel, A. (1872). Paléontologie ou description des animaux fossiles de la province d'Oran. Zoophytes. 5ème Fascicule, Spongiaires (Perrier, Oran), 256 pp.

Pomel, A. (1883). Classification méthodique et genera des échinides vivants et fossiles. Aldolphe Jourdan, Alger,131 pp. https://doi.org/10.5962/bhl.title.11272

Popenoe, W.P. (1937). Upper Cretaceous Mollusca from southern California. Journal of Paleontology, 11: 379-402.

Renz, C. (1930). Neuemittel kretazische Fossil vorkommen in Griechenland. Mémoires de la Société paléontologique Suisse (Abhandlungen der Schweizerischen Paläontologischen Gesellschaft), 49 (5): 1-10.

Rhalmi, M.; Pascal, A. \& Chellai, E. (2000). Lithobiostratigraphie, diagenèse et paléogéographie au Cénomanien supérieur-Turonien inférieur des bassins sud-atlasiques marocains. Géologie Alpine, 76: 135-149.

Robaszynski, F.; Caron, M.; Amédro, F.; Dupuis, C.; Hardenbol, J.; Gonzalez- Donoso, J.M.; Linares, D. \& Gartner, S. (1993). Le Cénomanien de la région de Kalaat Senan (Tunisie Centrale): biostratigraphie et interprétation séquentielle. Revue de Paléobiologie, 12 (2): 351-505.

Roemer, F. (1849). Texas. Adolph Marcus, Bonn, $495 \mathrm{pp}$.

Roemer, A. (1852). Die Kreidebildungen von Texas und ihre organischen Einschlüsse. Adolph Marcus, Bonn, 100 pp. https://doi.org/10.5962/bhl.title. 15015

Roney, R.O. (2013). Paleobiogeographical variation of Cretaceous Mecaster batnensis and Mecaster fourneli (Echinoidea: Spatangoida). Unpublished M. Sc. thesis, University of Tennessee, 203pp.

Rossi Ronchetti, C. \& Albanesi, C. (1961). Fossili Cenomaniani del Gebel Tripolitano. Rivista italiana di Paleontologia., 3: 251-318.

Ruault-Djerrab, M.; Ferré, B. \& Benkherouf, F. (2012). Étude micropaléontologique du Cénomano-Turonien dans la région de Tébessa (NE Algérie): implications paléoenvironnementales et recherche de l'empreinte de l'OAE2. Revue de Paléobiologie, 31 (1): 127-144.
Russo, A. (1958). lamellibranchi delle marne cenomaniane "in facies africana" della zona di Barcellona di Sicilia. Mem. Ist. Geol. Min. Univ. Padova, 20, 26 p.

Sadooni, F.N. (2005). The nature and origin of Upper Cretaceous basin-margin rudist buildups of the Mesopotamian Basin, southern Iraq, with consideration of possible hydrocarbon stratigraphic entrapment. Cretaceous Research, 26: 213-224. https://doi.org/ 10.1016/j.cretres.2004.11.016

Savornin, J. (1931). La géologie algérienne et nord africaine depuis 1830. Publlication du Service de Carte d'Algérie. 1vol., 395 pp.

Say, T. (1820), Observations on some species of zoophytes, shells, etc. principally fossil: American Journal of Science, ser. 1, 2: 34-45.

Schloteim, E.T. (1813). Beiträge zur Naturgeschichte der Versteinerungen in geognostischer Hinsicht. Leonhard's Taschenbuch der Mineralogie, 7: 1-134.

Schulze, F.; Kuss, J. \& Marzouk, A.M. (2005). Platform configuration, microfacies and cyclicites of the upper Albian to Turonian of west-central Jordan. Facies, 50: 505-527. https://doi.org/10.1007/s10347004-0032-7

Seeling, J. \& Bengtson, P. (1999). Cenomanian oysters from the Sergipe Basin, Brazil. Cretaceous Research, 20: 747-765. https://doi.org/10.1006/cres.1999.0190

Seguenza, G. (1882). Studi geologici e paleontologicisul Cretaceo medio dell'Italia meridionale. Attidella Accademia Nazionale dei Lincei, serie 3: 65-214.

Sharpe, D. (1850). On the secondary district of Portugal which lies on the north of the Tagus. Quarterly Journal of the Geological Society, 6: 135-195. https://doi. org/10.1144/GSL.JGS.1850.006.01-02.18

Sharpe, D. (1853-1857). Description of the fossil remains of Mollusca found in the Chalk of England. I, Cephalopoda. Palaeontographical Society Monographs, 68: p. 1-26 (1853); 27-36 (1855); 37-68 (1857).

Slami, R.; Salmi-Laouar, S.; Ferré, B.; Aouissi, R. \& Benkherouf-Kechid, F. (2018). Biostratigraphie, géochimie et réponse des composantes microfauniques aux variations environnementales au passage Cénomanien-Turonien à Thénièt El Manchar (Monts de Bellezma, Batna, NE, Algérie). Estudios Geológicos, 74(1), e077. https://doi.org/10.3989/egeol.43039.472

Slami, R. (2014). Biostratigraphie et paléoenvironnement de la limite Cénomano-Turonienne de la région de Batna, à partir de l'étude de la faune et la microfaune. Mémoire de Magister, Université Hadj Lakhdar, Batna, 229 pp.

Smith, A.B. (1995). Late Campanian-Maastrichtian echinoids from the United Arab Emirates-Oman border region. Bulletin of the Natural History Museum, Geology series, 51: 121-240.

Smith, A.B. \& Wright, C.W. (1990). British Cretaceous echinoids. Part 2, Echinothurioida, Diadematoida and Stirodonta (1, Calycina). Palaeontographical Society Monographs, 143: 101-198. 
Smith, A.B. \& Bengtson, P. (1991). Cretaceous echinoids from north-eastern Brazil. Fossils and Strata, 31: 1-88.

Soares, A.F. (1966). Estudo das formações pós-jurássicas da região de entre Sargento-Mor e Montemo-oVelho (Margem direita do Rio Mondego). Revista Faculdade de Ciências, Universidade de Coimbra XL. Memórias e Noticias. Vol. 62, 1-343.

Sowerby, J. \& Sowerby, J. de C. (1812-1846). The mineral conchology of Great Britain; or, coloured figures and descriptions of those remains of testaceous animals or shells which have been preserved at various times and depths in the earth. B. Meredith, London, 803 pp.

Stenzel, H.B. (1971). Oysters. In: Treatise on Invertebrate Paleontology (Moore., R. C. Ed.,), Part N, Mollusca 6(3), Bivalvia. Geological Society of America and University of Kansas Press, Boulder and Lawrence, N953-N1224,

Stoliczka, F. (1865). Eine Revision der Gastropoden der Gosauschichten in den Ostalpen. Sitzungsberichte der Kaiserlichen Akademie der Wissenschaften, Mathematisch- Naturwissenschaftliche Klasse, 52: 104-223.

Stoliczcka, F. (1870-71). Cretaceous fauna of southern India: The Pelecypoda, with a review of all known genera of this class, fossil and recent. Paleontologica Indica, 6(3): 1-557.

Tentor, A. (2007). Stratigraphic observations on Mount Brestori (karst of gorizia, Itali). Natura Nascosta, 35: $1-23$.

Thomas, H.D. \& Omara, S. (1957). The Cenomanian compound coral, Aspidiscus cristatus (Lamarck) from Nezzazat, western Sinai. Geological Magazine, 94: 151-155. https://doi.org/10.1017/S0016756 800068424

Trevisan, L. (1937). La fauna e i giacimenti del Cenomaniano di facies africana della Sicilia occidentale. Mémoire dell'Instituto Geologico della Universita di Padova, 12: 1-132.

Van Hoepen, E.C.N. (1929). Die Krytfauna van Soeloeland. I. Trigoniidae: Paleontologiese Navorsing van die Nasionale Museum, 1: 1-38.

Vila, J.M. (1980). La chaine alpine d'Algérie orientale et des confins algéro-tunisiens. Thèse Doctorat Sciences Naturelles, Université Pierre et Marie Curie, Paris VI, 2, 665 pp.

Vivière, J.L. (1985). Les ostracodes du Crétacé supérieur (Vraconien à Campanien basal) de la région de Tébessa (Algérie du Nord-Est): Stratigraphie, paléoécologie, systématique. Thèse Doctorat 3ème cycle, Université Pierre et Marie Curie, Paris VI, 261 pp.

Vyalov, O.S. (1936). Sur la classification des huîtres: Comptes Rendus (Doklady) de l'Académie des Sciences de l'URSS, Nouv Ser., 4(13), 1(105): 17-20.

Waller, T.R. (1978). Morphology, morphoclines and a new classification of the Pteriomorphia (Mollusca: Bivalvia). Philosophical Transactions of the Royal Society of London, B 284: 345-365. https://doi. org/10.1098/rstb.1978.0072
Watson, H. (1930). On the anatomy and affinities of Plicatula: Proceedings of the Malacological Society of London, 19: 25-31.

Wiese, F. \& Schulze, F. (2005). The Upper Cenomanian (Cretaceous) ammonite Neolobites vibrayeanus (D'Orbigny, 1841) in the Middle East: taxonomic and palaeoecologic remarks. Cretaceous Research, 26: 930-946. https://doi.org/10.1016/j. cretres.2005.06.005

Wilkes, J. (1810). Conchology. In: Encyclopaedia Londinensis; or, Universal Dictionary of Arts, Sciences, and Literature. J. Adlard, London, 14-41.

Woods, H. (1913). Ostreidae, Radiolitidae, additions, distribution, bibliography, Index: A Monograph of the Cretaceous Lamellibranchia of England, Vol. II, Part IV, Paleontographical Society, 341-473.

Wright, C.W. \& Kennedy, W.J. (1987). The Ammonoidea of the Lower Chalk. Part 2. Monograph of the Palaeontographical Society, 139: 127-218.

Wright, C.W.; Callomon, J.H. \& Howarth, M.K. (1996). Cretaceous Ammonoidea. In: Treatise on Invertebrate Paleontology, Part L, Mollusca 4 revised (Kasler, R.L., Ed.), Geological Society of America and University of Kansas, Boulder and Lawrence, USA, 1-262.

Yahiaoui, A. (1990). La partie inférieure de la série marnocalcaire du Crétacé supérieur (Cénomanien supérieur à Coniacien inférieur) entre Batna et El Kantara (Algérie orientale): Stratigraphie, Sédimentologie et Paléogéographie, Thèse de Doctorat, Université de Nancy I, 207 pp.

Zaghbib-Turki, D. (1989). Les échinides indicateurs des paléoenvironments, un exemple dans le Cénomanien de Tunisie. Annales de Paléontologie (Invertébrés), 75(2): 63-81.

Zakhera, M.S. (2011). Cenomanian-Turonian rudists from Western Sinai, Egypt: Systematic paleontology and paleoecology. Géobios, 44: 409-433. https://doi.org/ 10.1016/j.geobios.2010.10.004

Zakhera, M.S. (2002). Upper Cretaceous (Cenomanian-Maastrichtian) gastropods from west of the Gulf of Suez, Egypt. Neues Jahrbuch für Geologie und Paläontologie, Abhandlungen, 225: 297-336.

Zaoui, D.; Tchénar, S.; Benyoucef, M.; Meister, C.; Adaci, M.; Piuz, A.; Mebarki, K.; Bensalah, M.; Gabani, A. \& Mahboubi, M. (2016). Le Cénomano-Turonien dans la Hamada du Tinrhert (Sahara, Algérie): résultats préliminaires. Revue de Paléobiologie, 35 (2): 541-559.

Zittel K.A. (1873). Paläontologische Studien über die Grenzschichten der Jura und Kreideformation. 3. Die Gastropoden der Stramberger Schichten. Paläontologische Mitteilungen aus dem Museum des Königlich Bayerischen Staates, 2: 311-490.

Zittel K.A. (1884). Handbuch der Palaeontologie. Abt. I, Palaeozoologie, Bd. II. Mollusca und Arthropoda. München, Leipzig, 329-522. 